\title{
WILEY-VCH
}

\section{Magnetic Actuation Methods in Bio/Soft Robotics}

Nafiseh Ebrahimi, Chenghao Bi, David J. Cappelleri, Gastone Ciuti, Andrew T. Conn, Damien Faivre, Neda Habibi, Alexander Hošovský, Veronica Iacovacci, Islam S. M. Khalil, Veronika Magdanz, Sarthak Misra, Chytra Pawashe, Rasoul Rashidifar, Paul Eduardo David Soto Rodriguez, Metin Sitti, Zoltan Fekete, Amir Jafari*

N. Ebrahimi,

GRA with Advanced Robotic Manipulators (ARM) lab, Department of Mechanical Engineering, University of Texas at San Antonio UTSA, One Circle, San Antonio, 78249, USA.

Prof. D. J. Cappelleri, C. Bi,

Associate Professor with School of Mechanical Engineering, Purdue University, 585 Purdue Mall, 47907, USA.

Prof. G. Ciuti,

Associate Professor with the BioRobotics Institute, Santa'Anna School of Advanced Studies,

Piazza Martiri della Libertà, 33, 56127 Pisa PI, Italy.

Dr. A. T. Conn,

Researcher with Bristol Robotics Laboratory, University of Bristol,

T Block, University of the West of England, Frenchay Campus, Bristol, BS16 1QY, United Kingdom.

Dr. D. Faivre, P. E. D. S. Rodriguez,

Researcher with Aix Marseille University, CEA, CNRS, BIAM, 13108 Saint Paul-Lez-Durance, France

Dr. N. Habibi,

Program Coordinator with Northwest Vista College, 3535 N Ellison Dr., San Antonio, TX 78251, USA.

Dr. A. Hošovský,

Researcher with Technical University of Kosice - Technicka univerzita v Kosiciach,

Letná 1/9, 04001 Košice, Slovakia.

Dr. V. Iacovacci

Researcher with the BioRobotics Institute, Santa'Anna School of Advanced Studies,

Piazza Martiri della Libertà, 33, 56127 Pisa PI, Italy.

Prof. I. S. M. Khalil,

Assistant Professor with Biomechanical Engineering, Faculty of Engineering Technology, University of Twente, Five, Drienerlolaan, 7522 NB Enschede, Netherlands.

Dr. V. Magdanz,

Postdoc with Chair of Applied Zoology, Faculty of Biology, Zellescher Weg 20b,

01069 Dresden, Germany.

Professor. S. Misra,

Professor with Biomechanical Engineering, Faculty of Engineering Technology, University of Twente, Five, Drienerlolaan, 7522 NB Enschede, Netherlands.

Dr. C. Pawashe,

Intel Corporation, Portland, Oregon Area, USA. 


\section{WILEY-VCH}

R. Rashidifar, GRA with Center for Advanced Manufacturing and Lean Systems, Department of Mechanical Engineering, University of Texas at San Antonio UTSA, One Circle, San Antonio, 78249, USA.

Prof. M. Sitti,

Professor with Max Planck Institute for Intelligent Systems, Heisenbergstraße 3, 70569 Stuttgart, Germany.

Dr. Z. Fekete,

Researcher with Faculty of Information Technology and Bionics, Pázmány Péter Catholic University, Budapest, Práter u. 50/A, 1083 Hungary.

Professor A. Jafari,

Assistant professor with Advanced Robotic Manipulators (ARM) lab, Department of Mechanical Engineering, University of Texas at San Antonio UTSA, One Circle, San Antonio, 78249, USA.

E-mail: amir.jafari@utsa.edu

Keywords: Magnetic microrobots, magnetic micro-manipulation, bio-inspired, Magnetotactic bacteria, Magnetically-guided capsule endoscopy.

Abstract_Many fundamentally different ways of actuation have been used in robots with magnetic actuation having a number of advantages in possible realization of tetherless robots through wireless energy transfer, precise positioning or insensitivity to perturbations by bio-chemical fluids. These robots can be driven by field gradients, oscillating or rotating fields provided by permanent magnets of electromagnetic coils and generally falling under the category of torque- or force-driven magnetic actuation. Both approaches can be then used for designing robots with different types of locomotion like swimming and rolling/tumbling robots operating on surfaces. Using the specific properties of magnetic actuation, it is possible to address the problems of controlling miniature devices in confined spaces within the human body, ranging from helical swimming robots and microgrippers to steering catheters and endoscopes. This review provides a summary of recent research in magnetically actuated bio-robots together with relevant applications in biomedical area and discusses future prospects of this way of actuation for possible improvements in performance of different types of biorobots. 


\section{WILEY-VCH}

\section{Introduction}

Magnetically actuated robots have been being developed for numerous applications of minimally invasive diagnosis and surgical intervention in many regions of body including abdomen, heart, brain, eye, ear and vascular system. These potential broad applications contain diagnostic imaging, inserting implants, drug delivery and biopsy. The magnetically actuated devices are being used for aforementioned purposes works based on different functions like swimming or bacteria propelled microrobots, catheters, capsule endoscopes and robotics. They also varies in size ranging from submillimeter to tens of centimeters. These devices are functioning based on the same physical principles although the design and implementation seems different and each device has its unique challenges. $^{[1,2]}$

Traditional robots are fast and precise systems, based on rigid-body mechanisms that enabled them to accurately control the motion and to apply torques and forces on joints and tips respectively. Since medical robots become smaller and penetrate deeper into the body of patients through its natural pathways, incorporating stiff components and rigid mechanical couplings to the outside environment in the robot's design have become infeasible. Hence, it has caused the exploration of new techniques for removing the mechanical coupling and emerging the concept of wireless force generation and transmission. Since magnetic actuators are able to be driven wirelessly by magnetic field, exploiting the concept of magnetism to generate force and torque for the new generation of medical robotics has been considered and proved itself as a promising technique that is capable of providing both needed power and precise control of the robotic device. ${ }^{[2,3]}$ In this new approach of magnetic actuation, the system could be built using permanent magnet or electromagnetic system which both represent sufficient capability of driving and propulsion of miniature robotic devices like helical swimming microrobots, steering catheters and continuum manipulators, launching pumps of implanted artificial heart and navigating the capsule endoscopes inside the human body. ${ }^{[2,4]}$ These magnetic instruments have the ability to reduce the invasiveness of medical interventions and diagnoses, as they can get rearranged inside the abdominal area without the necessity of dedicated port during the procedure. In such instruments on-board actuators can be implemented to accomplish a controlled and replicable motion at the interface with the body. ${ }^{[5]}$ Moreover, MRI (Magnetic Resonance Imaging) scanners also have been used to provide robot tracking and control of the devices inserted into the body for navigation along the vascular system or needle biopsy and tissue ablation. There are some common challenges for such kind of magnetic actuation systems specially applicable in medical devices like generating appropriate force and torques, precise localization and navigation of the device, minimization of the magnetic actuation device footprint, and optimization of the operational workspace. ${ }^{[6]}$

Due to the significance of the magnetic actuation method in soft/bio robots applicable in medical diagnostic and surgical interventions, we reviewed the latest advances happened in recent years to provide a comprehensive resource for future researchers interested in this field.

In the following sections, we are going to discuss the broad variety of magnetically actuated robotic devices, which are mostly bio inspired and then review their applications specifically in biorobots.

\section{Bio-inspired Magnetic Microrobots [Fekete, Andrew T. Conn]}

Discovery of the propelling motion of microorganisms relying on flagella and cilium dates back to the $19^{\text {th }}$ century. Much later in 1973, Berg proved that E.Coli bacteria use molecular motors to rotate their helical flagella. ${ }^{[7]}$ To change position in fluids, such microscale organisms need a 


\section{WILEY-VCH}

special strategy as low Reynolds number (typically between $10^{-2}$ to $10^{-5}$ ) in their environment makes reciprocal motion ineffective. Instead, they achieve propulsion by propagating traveling waves along their flagella. To mimic this concept widely exploited in nature, a number of synthetic microrobots have been proposed mostly relying on planar, helical and cilia based microstructures. In this section, each type will be concisely described and also shown in Figure 1 including examples from the literature.

Planar locomotion can be triggered by a flexible flagellum, which requires only one dimensional structure. Mimicking the beating of spermatozoa flagellum, planar waveform can be generated. Artificial planar propellers are comprised of flexible sheets attached to magnetic head and actuated using oscillating magnetic field. Early prototypes of planar micro-swimmers were demonstrated by Dreyfus et al in 2005 , when a flexible tail made of self-assembled magnetic beads was used to evoke drag forces. ${ }^{[8]}$ Their method was further improved to connect the beads with DNA strands, and demonstrated the drag of a red blood cell. ${ }^{[9]}$ Later, developments in the technical implementation of this configuration paved the way to optimize the efficiency of propulsion. Magnetically driven locomotion of flexible $\mathrm{Au} / \mathrm{Ag} / \mathrm{Ni}$ nanowires, with a gold 'head' and nickel 'tail', linked by a partially dissolved and weakened silver bridge was presented by Gao. ${ }^{[10]}$ Rigid magnetic Ni head with flexible silver tail was produced by template directed electrodeposition. ${ }^{[11]}$ Bending of its flexible tail was achieved by precessing magnetic field around the direction of movement. Optimizing the magnetization profile, Diller et al proposed dispersed ferromagnetic powder in an elastomer matrix, and showed propagation of bending waves generated by external rotating magnetic field. ${ }^{[12]}$ Jang et al demonstrated a robot comprising an elastic eukaryote-like polypyrrole tail and rigid magnetic Ni links connected by flexible polymer bilayer hinges. Their pioneering work showed that planar undulation in nanowire-based chains propelled by planaroscillating magnetic field is possible. ${ }^{[13]}$ Combination of multistep electrodeposition and selective 


\section{WILEY-VCH}

etching has been used to produce undulating slender micropropellers with multiple magnetic links. ${ }^{[14]}$ Such distributed magnetization in the structure allows the propagation of bending waves along the flagellum, circumventing the limitation of typical one-sided, synchronous bending of artificial flagella.

Helix-like flagella of several bacteria (like E.Coli) are used as molecular motors to propel these microorganisms. Artificial helical microrobots uses rotating magnetic field for propulsion, without on-board rotary motor. Conceptually, they consist of a rigid helical tail attached to a soft ferromagnetic head, eventually diametrically magnetized. The swimmer is rotated around its helical axis, while the direction of locomotion is perpendicular to the plane of rotation. These structures can be propelled forwards or backwards by simply changing the direction of rotation of the magnetic field. To produce helical structures for magnetic locomotion, several microfabrication method has been proposed in the last decade. The so-called self-scrolling method as the pioneering work of Bell and colleagues was demonstrated to control the internal stress of thin film multi-layered ribbons ending in a square shaped, deposited Ni head. ${ }^{[15,16]}$ Glancing angle deposition (GLAD) was first proposed by Ghosh et al. ${ }^{[17]}$ They grow helical pillars on spherical seeds during rotation of a tilted stage in an evaporation chamber, while cobalt layer deposited and permanently magnetized perpendicular to the helical axis was performed afterwards. Template assisted electrodeposition processes have been utilized to produce helical magnetic microstructures. Khalil et al have utilized electrospinning with a polymer solution (polystyrene in dimethyl formamide) and iron-oxide nanoparticles to create a robotic sperm that can swim faster than micro-swimmers that rely planar wave propulsion. ${ }^{[18]} \mathrm{Li}$ et al has demonstrated how Pd nanospring as templates can be used to synthetize helical microstructures after Ni deposition and selective Pd dissolution. ${ }^{[19]}$ 3D printed micromolds have been also applied for template-assisted electrodeposition in the work of Alcantara et al, where helical Fe-based micro-swimmers were 


\section{WILEY-VCH}

proposed to reduce cytotoxicity of the metallic parts. ${ }^{[20]}$ Soft lithography and micromolding processes were utilized by Ye et al to demonstrate that multiple flagella on one microrobot can generate a higher propulsive force and therefore faster swimming. ${ }^{[21]}$ GLAD and template assisted deposition allows the formation of structure of sub-micrometer scale, while earlier approaches ended in structures of a few or tenth of micrometer in length. This is especially important as microscale propellers are able to swim in Newtonian fluids, however, apart from urine and CSF, biological fluids are non-Newtonian and only nanoscale robots having a filament diameter below $70 \mathrm{~nm}$ range can move. ${ }^{[22]}$ Template-based synthesis has been improved by using biological organs as replica. First, Schuerle et al used diacetylenic phospholipids as a template, and coated with a multi-alloy magnetic coating. ${ }^{[23]}$ Later, Yan exploited the helical structure of Spirulina platensis, and deposited magnetite precursors on its surface, which was followed by annealing treatment and reduction processing to create porous hollow magnetic microhelices. ${ }^{[24]}$

To transport micro objects, or particles, the helical body as a stand-alone structure needed further improvements. The rapid development of two-photon polymerization enabled to extend the basic helix with microholders to facilitate the stable manipulation of cargos. One of the early demonstration of such 3D machinery was reported by Tottori et al, who achieved the transportation of polysterene beads with a helical micromachine. ${ }^{[25]}$ Magnetic property of these polymer structures can be established with either evaporation after the laser writing process or simply adding magnetic microparticles to the photosensitive constituting material of the $3 \mathrm{D}$ patterned objects. ${ }^{[17,26]}$ Further advantage of the $2 \mathrm{P}$ polymerization was demonstrated by Barbot and coauthors, whose so-called roll-to-swim microrobot showed 3D navigation in a fluid medium combining three type of motions (rolling, swimming, spin-top) in a controlled fashion. ${ }^{\text {[27] }}$

Cilia are short, hair-like nanostructures covering the cell body. A well-known unicellular organism to mimic is Paramecium, which uses cilia to propel its body in the surrounding medium. Unlike 


\section{WILEY-VCH}

the undulatory motion of flagella, cilia exhibit flexural motion with a characteristic power stroke to drive fluid flow and a flexible recovery stroke. ${ }^{[28]}$ Cilia beat in an asynchronous motion that forms a metachronal wave. ${ }^{[29]}$ Artificial magnetic cilia, in contrast to its natural counterpart, moves simultaneously to achieve locomotion. Artificial cilia have been developed by forming magnetoelastic microstructures made from PDMS-ferromagnetic particle composites, which can be integrated on the surface of lab-on-chip devices to pump and mix fluids. ${ }^{[30,31]}$ Due to the synchronous beating of these hair-like appendages exposed to the global magnetic field, fluid propulsion is unsteady, therefore ciliary motion of microrobots is still limited. Besides theoretical difficulties, the first ciliary microrobot cruising with stroke motion was proposed in 2016. Kim et al designed a lithographically defined ellipsoidal body with cilias on its sides. ${ }^{[32]}$ Cilium of the two-photon polymerized structure were deposited with Ni. The power stroke and recovery stroke was programmed through a magnetic field of gradually changing direction. Numerical simulations of magnetic micro cilia has shown that different control strategies can generate the asymmetric motion of cilia, such as exploiting the buckling of a permanently magnetic film and using elastic coupling between a pair of magnetic bodies within a rotating magnetic field. ${ }^{[33,34]}$

Demonstrations of early in vitro applications of bioinspired magnetic microrobots are promising. Medina-Sanchez et al have shown the controlled transport of sperm cells with reduced motility aided by a synthetic microhelix propelled with rotating magnetic field. ${ }^{[35]}$ Functionalization of these micro-swimmers have also open up new perspectives in molecular delivery. The controlled release of drugs was achieved by ABF functionalized with liposomes. ${ }^{[36]}$ The advances in twophoton polymerization fostered further improvements in cargo delivery. A scaffold-like 3D helical microrobot was presented for three dimensional culturing and delivery of stem cells in vitro. ${ }^{[37]}$ Hollow microhelices with improved kinetic properties were proposed by Xin et al to targeted 


\section{WILEY-VCH}

delivery of nanoparticles and stem cells. ${ }^{[38]}$ Their special fabrication approach also relied on 2PP by helical scanning using femtosecond vortex beams produced by spatial light modulation.

More recently, efforts to facilitate the practical, in vivo use of magnetic microrobots have been made by several groups. Soft helical micro-swimmers composed of non-toxic photo-crosslinkable hydrogel gelatin methacryloyl (GelMA) rendered with magnetic nanoparticles is reported by Wang and co-authors. ${ }^{[39]}$ Their exemplary approach to use only biodegradable materials will be definitely a basic feature of future microrobots for biomedical purposes. The Nelson group presented the first in vivo demonstration of fluorescent tracking of magnetically actuated $A B F$ (functionalized with NIR probes) in the peritoneal cavity of mouse. ${ }^{[40]}$ Such advancement in the development of micro-swimmers are definitely necessary to move towards real medical applications.
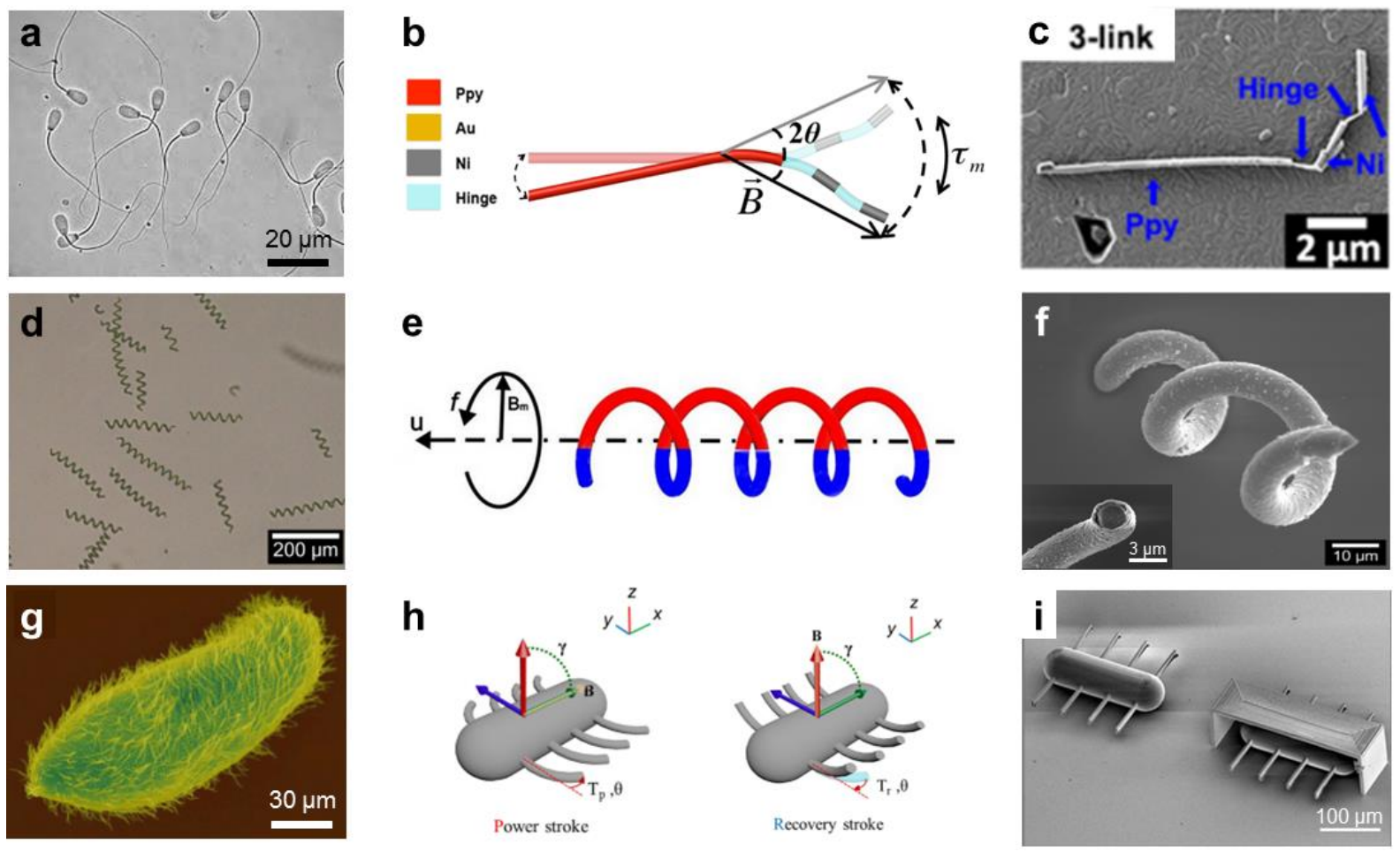

Figure 1. a) Microscopic view of mature spermatozoa, b) Schematic of a planar nano-swimmer comprising $\mathrm{n}$ elastic eukaryote-like polypyrrole tail and rigid magnetic nickel links connected by flexible polymer bilayer hinges. ${ }^{[13]}$ Undulatory motion is produced by magnetic field oscillation. c) SEM view of the 3-link nano-swimmer. ${ }^{[13]}$ d) Microscopic view of Spirulina bacteria. e) Schematic on the propulsion of helical microrobots. f) Biotemplated helical micro-swimmer. Inset picture shows cross-section of the helix before dissolving Spirulina bacteria as the biotemplate. ${ }^{[41]}$ 
g) Colored SEM micro-graph of a paramecium. h) Schematic on the two-stroke locomotion of a ciliary microrobot. i) Ciliary micro-swimmers fabricated by the combination of two-photon polymerization and subsequent metal deposition. ${ }^{[32]}$ Pictures b-c are reproduced from the work of Jang et al. ${ }^{[13]}$ Picture $f$ ) is reproduced from the paper of Gong et al. ${ }^{[41]}$ Pictures g-i) are reproduced from the article of Kim et al. ${ }^{[32]}$

\section{Untethered Magnetic Microrobots [Pawashe]}

In an ideal interpretation, an untethered microrobot can be imagined as a self-contained device, with all dimensions under $1 \mathrm{~mm}$, capable of mobility, sensing and reacting to the environment, and communicating to the outside world. Such a device would require an onboard power source, actuation system, sensory systems, and communication modules. Certainly, there exist centimeterscale robots that meet these criteria, but in the realm of the micrometer-scale, there currently does not exist sufficient miniaturization of components to have a fully self-contained microrobot.

In the current state-of-the-art, microrobots are more accurately described as the sub-millimeter sized wireless end-effector of a larger scale system, which externally provides energy and control inputs. The use of magnetism has gained popularity for controlling such microrobots, which can be via an electromagnetic-coil system, or a system of moving permanent magnets. ${ }^{[42]}$ Other actuation mechanisms have also been studied, such as electrostatic, and thermal approaches. ${ }^{[43,44]}$ Magnetic actuation mechanisms offer advantages such as (1) creating relatively strong torques and forces at micro-scale, which can be used for 2D and 3D motion and orientation control, (2) the microrobots themselves can be relatively simple objects, which are robust to handling, and (3) in some implementations, the working environment does not need to be specialized.

Two classes of untethered magnetic microrobots in the literature, with all dimensions below $1 \mathrm{~mm}$, will be covered in this section: (1) microrobots that operate in 2D on surfaces, and (2) microrobots that operate in 3D in a fluid environment driven by magnetic field gradient pulling. Microrobots that are inspired from biology and nature are discussed as follows: 


\section{WILEY-VCH}

\subsection{Microrobots Operating on 2D Surfaces}

A simple way to move a magnetic object on a surface using an external magnetic field is to apply a magnetic field gradient, which results in a force applied on that magnet. This force is proportional to the volume of the magnet. When the magnet is at the micro-scale, i.e. a magnetic microrobot, area-based forces dominate volume-dependent effects. A microrobot can experience high surface friction due to interfacial adhesion, which can dominate the effect of magnetic forces, effectively making the microrobot "sticky" with the surface. Applying a sufficiently strong magnetic field gradient can result in a large enough force to overcome this friction. However, this can lead to high accelerations, and thus an uncontrollable microrobot, especially in gaseous environments with low damping. Instead, oscillatory actuation methods have been employed in the literature to control magnetic microrobot motion, which overcomes the friction effects in a controllable fashion.

Three sub-millimeter magnetic-based microrobots will be discussed, which employ unique actuation methods using oscillatory external inputs: the MagMite, the Mag- $\mu$ Bot, and the MagPier. ${ }^{[45-47]}$

\subsubsection{MagMites}

The MagMite is a magnetic microrobot whose motion is derived from wireless resonant magnetic actuation. ${ }^{[45]}$ Constructed using microfabrication techniques, it consists of a gold frame, a spring element, and two nickel soft magnetic bodies. One of these bodies is mobile and is attached to the spring element. The other body is stationary, and rests on the MagMite's frame. The overall dimensions of the MagMite are below $300 \mu \mathrm{m}$. Figure 2 shows the MagMite and its fabrication process. 


\section{WILEY-VCH}
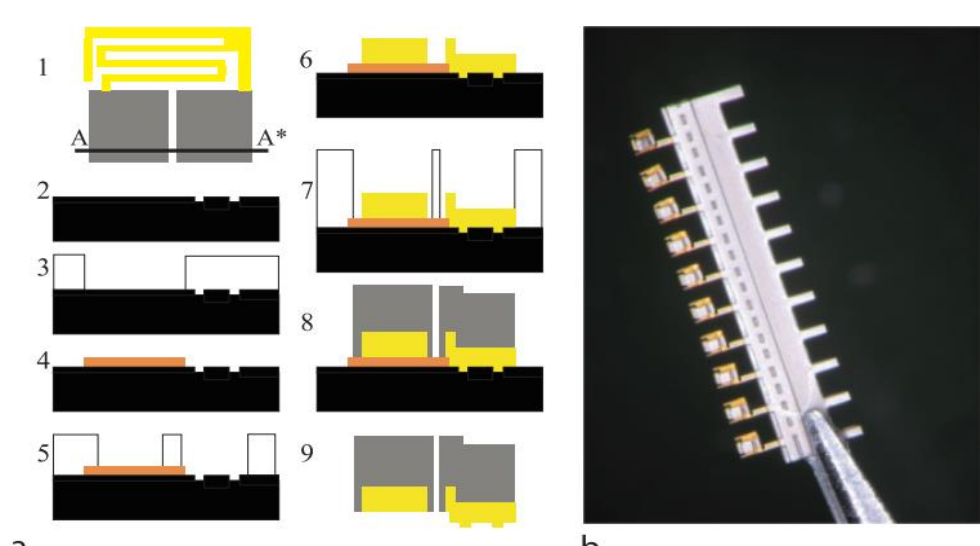

b

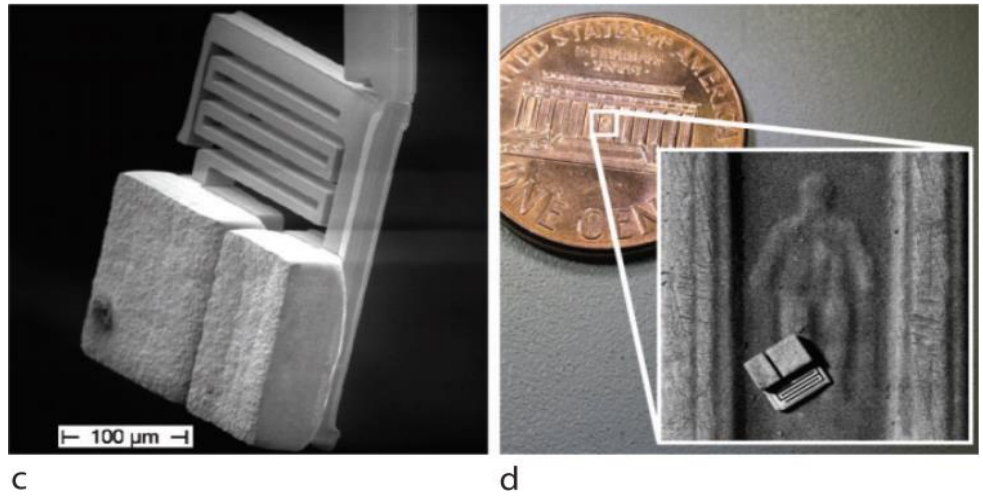

Figure 2. (a) Microfabrication process steps to fabricate MagMite, (b) a strip of released MagMite microrobots, (c) scanning electron micro-graph of a MagMite, (d) a MagMite on a US penny.

The MagMite achieves mobility by momentum transfer from its mobile magnetic body impacting the stationary magnetic body, which vibrates from the forces induced by oscillating external magnetic field gradients. These gradients are generated by a pair of Helmholtz coils, and the frequency of the field is specified to achieve mechanical resonance in the MagMite's mass-spring system. For a MagMite with symmetric magnetic bodies, this process would nominally result in a MagMite that vibrates in place. To achieve forward motion, a rectification signal is applied through the means of a downward force generated by electrostatic fields on a specialized surface, which allows the MagMite's friction with the surface be controlled. The rectifying signal is applied at the same frequency as the magnetic field, shifted 180 degrees out of phase. The result is that for one half of the oscillation cycle, there is no MagMite motion due to high friction, but in the other half, there is an increment in motion due to low friction. With driving frequencies in the $\mathrm{kHz}$ range, 


\section{WILEY-VCH}

continuous motion can be achieved. Orientation and turning is achieved by magnetic torques arising from the external magnetic fields.

The MagMite can achieve velocities of over $12.5 \mathrm{~mm} / \mathrm{s}$, and can move forward and backwards depending on the phase of the magnetic and electrostatic signals. Using a MagMite with asymmetric magnetic bodies, mobility can be achieved without the electrostatic clamping signal, although with a loss of efficiency and control. However, this allows the microrobot to operate on non-specialized surfaces, such as glass, bare silicon, or flat non-magnetic metals. In addition, the MagMite can operate in fluid environments, where motion is observed to be smoother due to the added damping of the environment.

Controlling multiple MagMites poses a challenge, because the driving magnetic fields are approximately uniform throughout the workspace. To select an individual MagMite, each is designed with different resonant frequencies. Through the application of time-division multiplexed signals, each individual MagMite can be independently controlled by taking turns moving. Figure 3 shows an example of two MagMites independently moving on a surface.
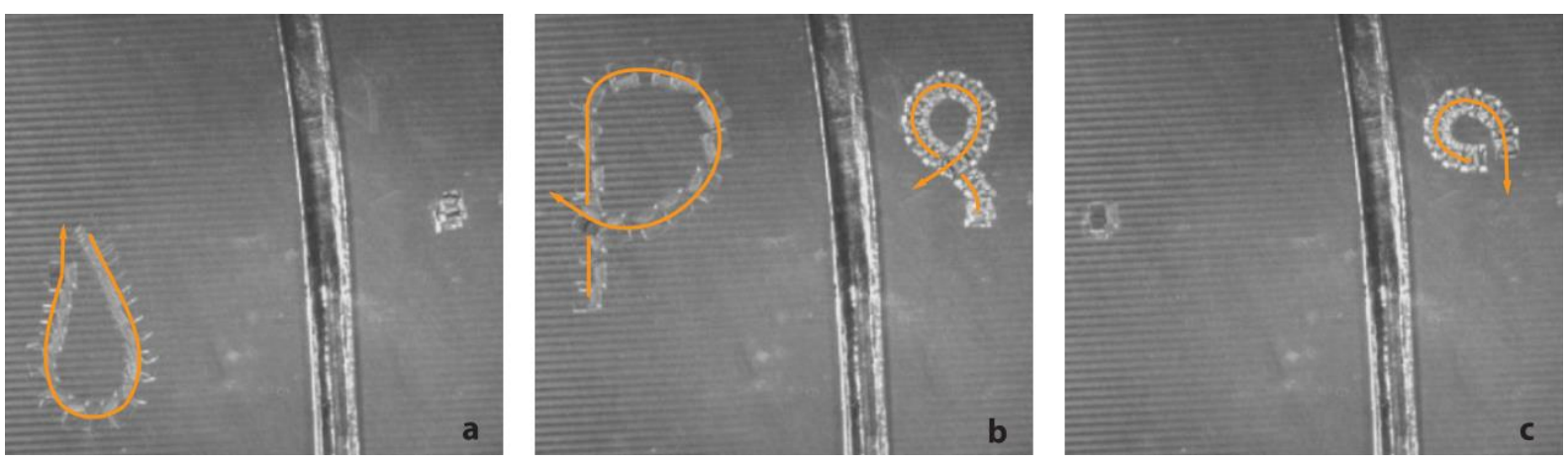

Figure 3. Two MagMites independently operating on the same surface, with motion paths illustrated. MagMites are separated by a polymer wall.

In an effort to reduce the fabrication complexity of the MagMite, the PolyMite was developed, based on similar operation principles as the MagMite. ${ }^{[48]}$ The PolyMite is built using SU8 polymer, with electrodeposited CoNi alloy to form the resonant magnetic bodies, shown in Figure 4. 


\section{WILEY-VCH}

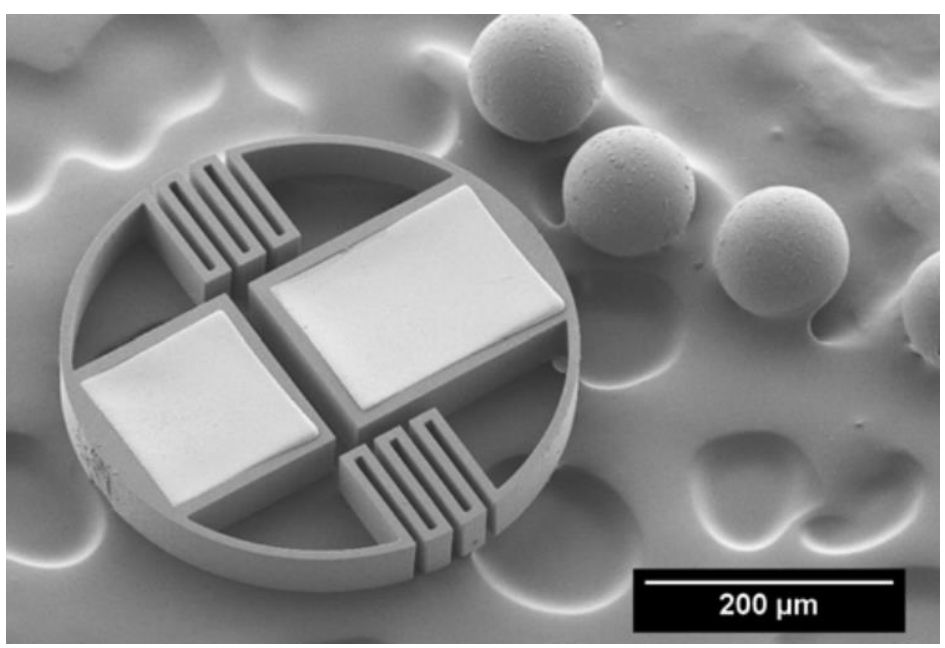

Figure 4. Scanning electron micro-graph of a PolyMite, with three polystyrene beads adjacent.

\subsubsection{Mag- $\mu$ Bots}

The Mag- $\mu$ Bot is a magnetic microrobot that achieves mobility by a stick-slip rocking motion mechanism. ${ }^{[46]}$ The microrobot itself is a simple permanent or soft magnet, fabricated to have submillimeter dimensions. It can be fabricated using laser micro-machining, or by using a polymerbased casting process, which allows it to be formed into arbitrary shapes. ${ }^{[49]}$ Being constructed out of bulk metallic material without delicate parts, the Mag- $\mu$ Bot is robust to handling.

External magnetic fields of 1-10 mT generated by five or six coils produce the necessary signal to locomote the Mag- $\mu$ Bot. Magnetic fields induce torques, which are relatively strong at this scale, and provide orientation as well as induce a rocking motion in the Mag- $\mu$ Bot. This rocking motion has a rotation axis parallel to the surface, which allows the Mag- $\mu$ Bot to intermittently break static friction, especially if the rocking motion is rapid in one half cycle of oscillation (a sawtooth waveform is typical). Combined with a magnetic field gradient to apply force, the Mag- $\mu$ Bot achieves controlled motion upwards of $10 \mathrm{~mm} / \mathrm{s}$, as it sticks and slips across the surface. It can operate on arbitrary surfaces, such as glass or silicon, and can also operate on rough surface such on the surface of a coin (Figure 5). The Mag- $\mu$ Bot can operate in air and liquid environments, 


\section{WILEY-VCH}

which has been observed to significantly affect motion characteristics; the added damping of a viscous liquid, such as silicone oil, leads to smoother and more controlled motion.

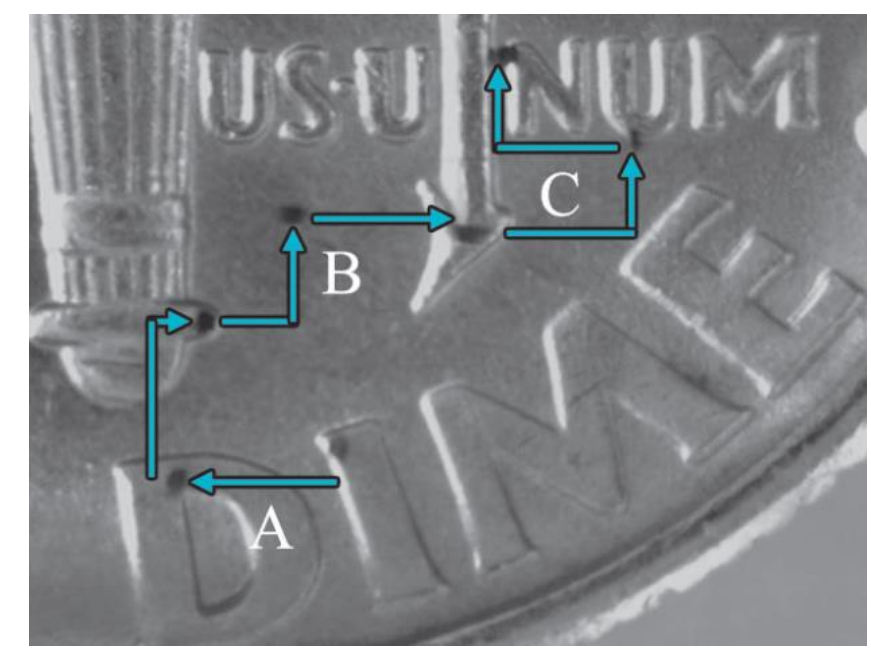

Figure 5. Frames from a video of a NdFeB Mag- $\mu$ Bot traversing on a US dime, which can move over smooth and bumped regions of the dime.

Control of multiple Mag- $\mu$ Bots has been demonstrated with two different approaches, with the constraint of having an approximately uniform driving magnetic fields throughout the workspace. In the first approach, a specialized surface provides an electrostatic anchoring signal, which can freeze Mag- $\mu$ Bots spatially. ${ }^{[46]}$ This allows multiple Mag- $\mu$ Bots to move independently of each other by taking turns. This electrostatic surface also has been used to assemble and disassemble multiple microrobotic modules (here called Mag- $\mu$ Mods), demonstrating the ability to create reconfigurable micro-scale constructs, which are also mobile using the same stick-slip mechanism as the Mag- $\mu$ Bot (see Figure 6 for the assembly, motion, and disassembly of eight Mag$\mu$ Mods). ${ }^{[50]}$ 


\section{WILEY-VCH}
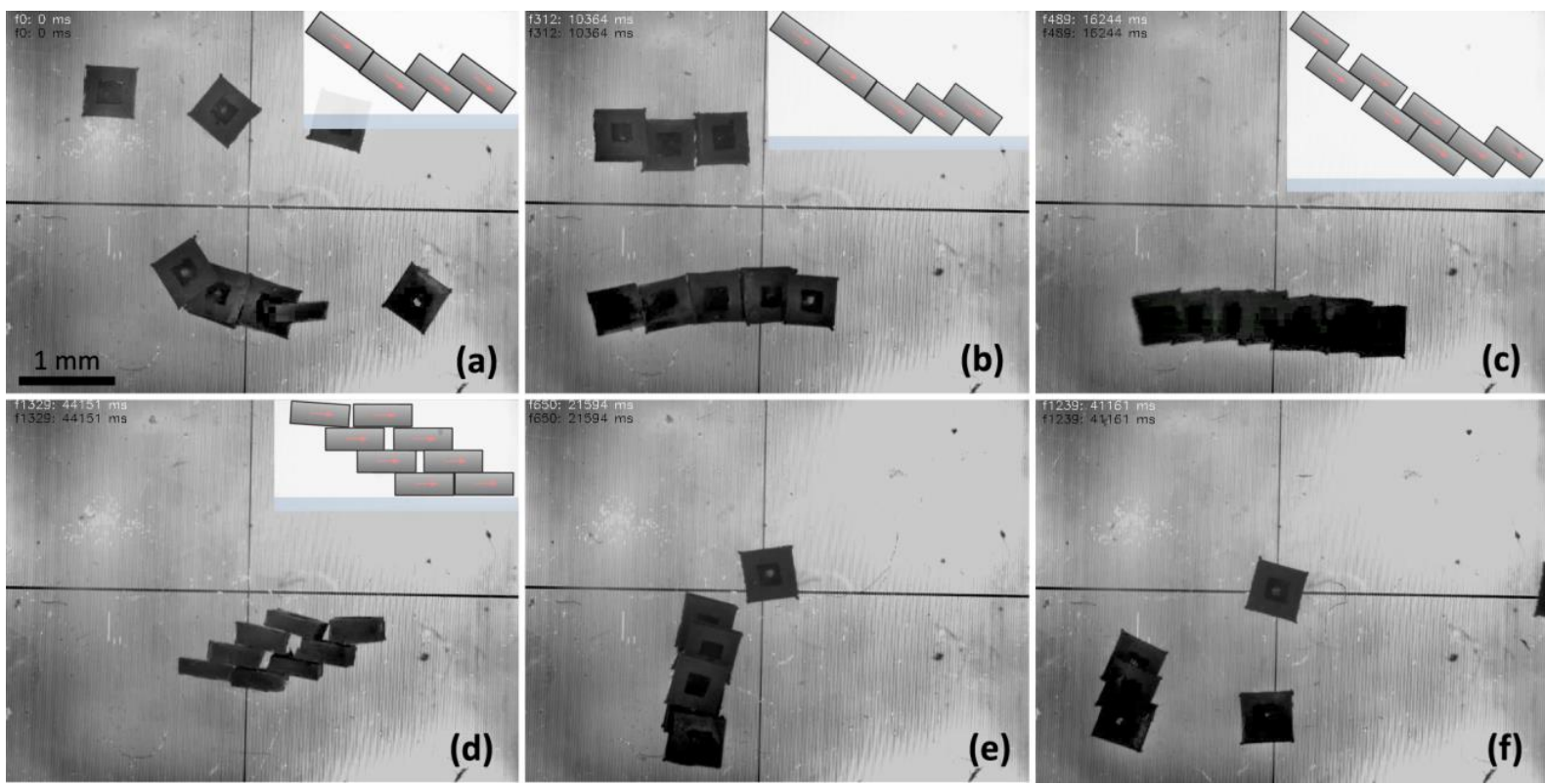

(d)
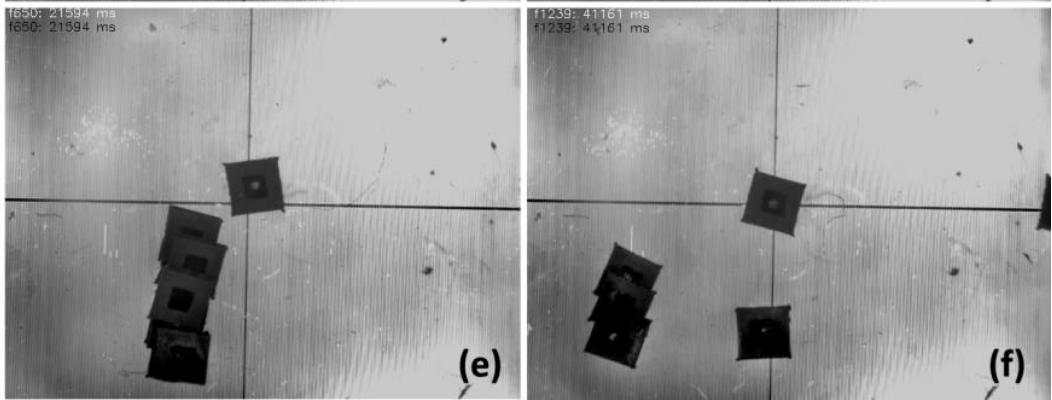

Figure 6. Eight Mag- $\mu$ Mods demonstrating assembly, disassembly, and mobility on an electrostatic surface. (a)-(d) shows assembly and mobility of individual Mag- $\mu$ Mods; inset shows schematic side-view. (e)-(f) shows disassembly and reconfigurability of the Mag- $\mu$ Mods.

The second approach to controlling multiple Mag- $\mu$ Bots obviates the need for a specialized surface. This approach relies on heterogenous microrobots, which can have varying dimensions and/or different internal magnetizations. ${ }^{[51]}$ By varying the frequency and amplitude of the applied magnetic field, different sets of Mag- $\mu$ Bots would react with motion. With an appropriate sequence of driving signals, these Mag- $\mu$ Bots can be independently positioned. Figure 7 demonstrates this approach, where three Mag- $\mu$ Bots were controlled on a non-specialized surface. 


\section{WILEY-VCH}

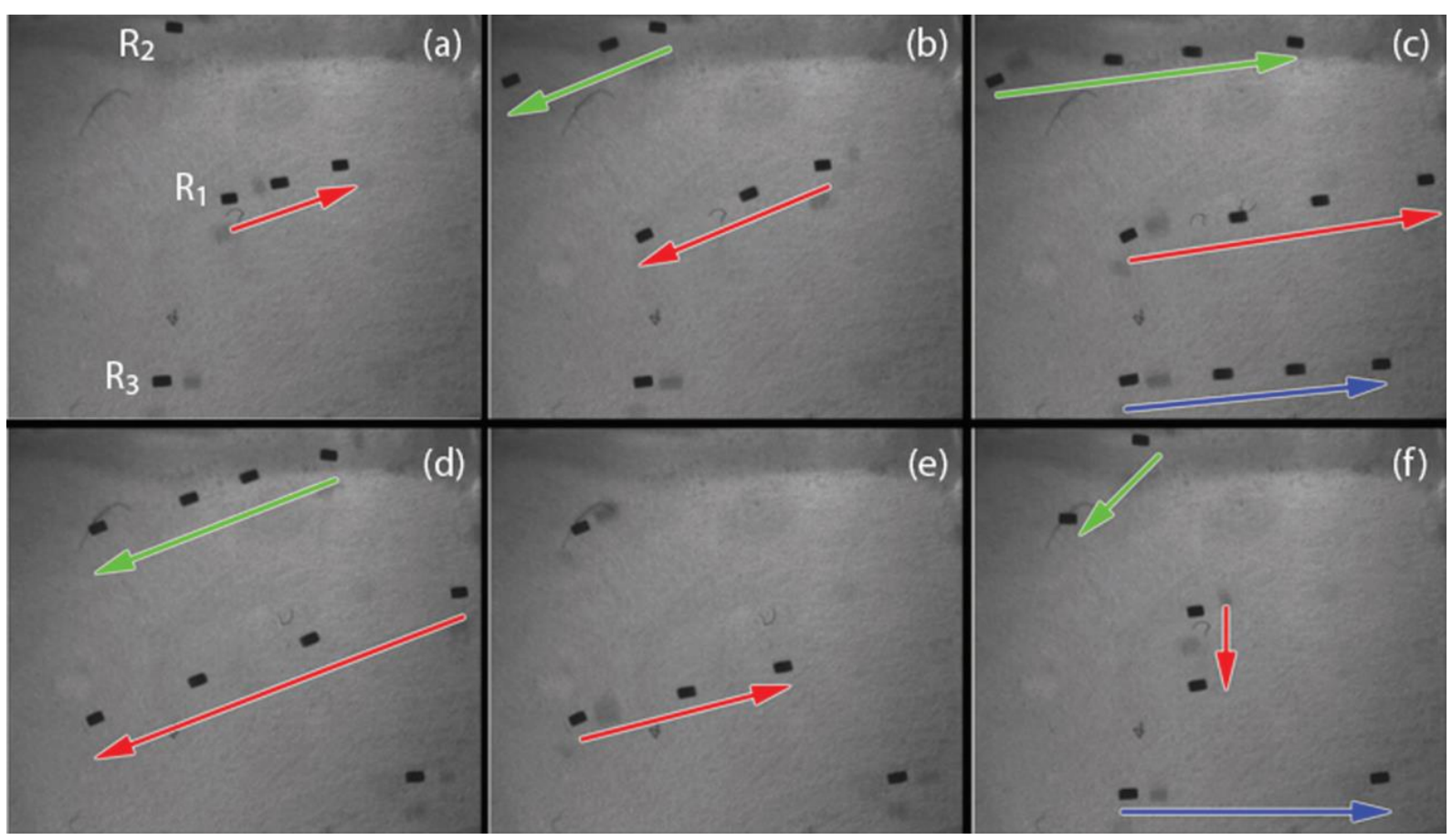

Figure 7. Frames from a video of three heterogeneous Mag- $\mu$ Bots, R1, R2, and R3, operating on a non-specialized glass surface, demonstrating independent positioning. Arrows indicate motion of each individual Mag- $\mu$ Bot in sequence shown.

\subsubsection{MagPieR}

MagPieR is a hybrid microrobot whose motion is based on magnetic and piezoelectric principles. ${ }^{[47]}$ Its body consists of a piezoelectric material, with a magnetic nickel layer on top, shown in Figure 8, and is fabricated to be under $400 \mu \mathrm{m}$ in all dimensions. This microrobot is specifically designed to achieve high velocities, upwards of $600 \mathrm{~mm} / \mathrm{s}$.

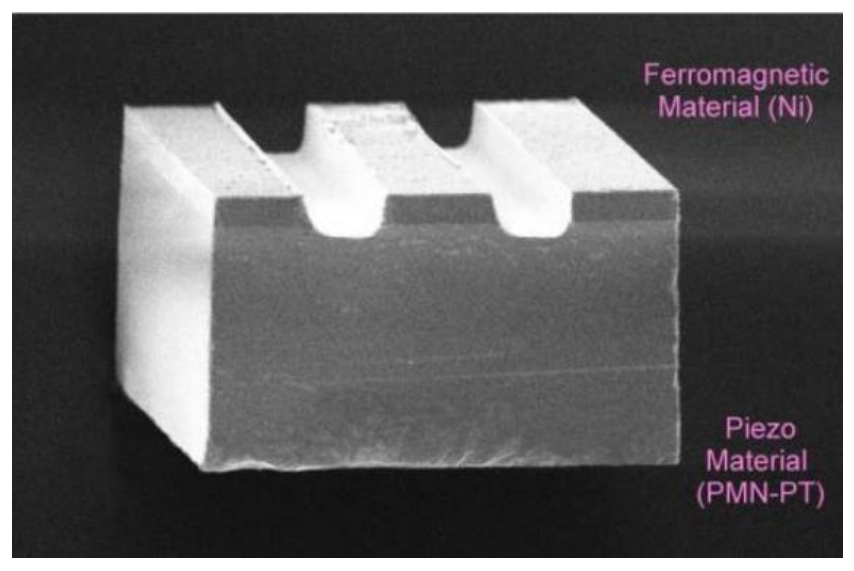




\section{WILEY-VCH}

Figure 8. Scanning electron micro-graph of a MagPieR microrobot, consisting of a piezoelectric material and a ferromagnetic material. Trenches shown are $50 \mu \mathrm{m}$ wide.

The MagPieR achieves mobility by a combination of external magnetic and electrostatic driving fields. Magnetic field gradients of magnitude approximately $5 \mathrm{mT} / \mathrm{mm}$ apply a force on the MagPieR, however this is insufficient to induce motion due to the high friction with the surface. To break the friction, an electric-field waveform is applied across the MagPieR to induce piezoelectric strain in the piezoelectric material. This is achieved by the specialized environment that the MagPieR must reside in, which is effectively inside the dielectric of a capacitor. Impulse waveforms of $300 \mathrm{~V}$ and $100 \mathrm{~Hz}$ has been shown to break the MagPieR's friction with the surface, allowing the magnetic field gradients to propel the microrobot in the desired direction (see Figure 9).

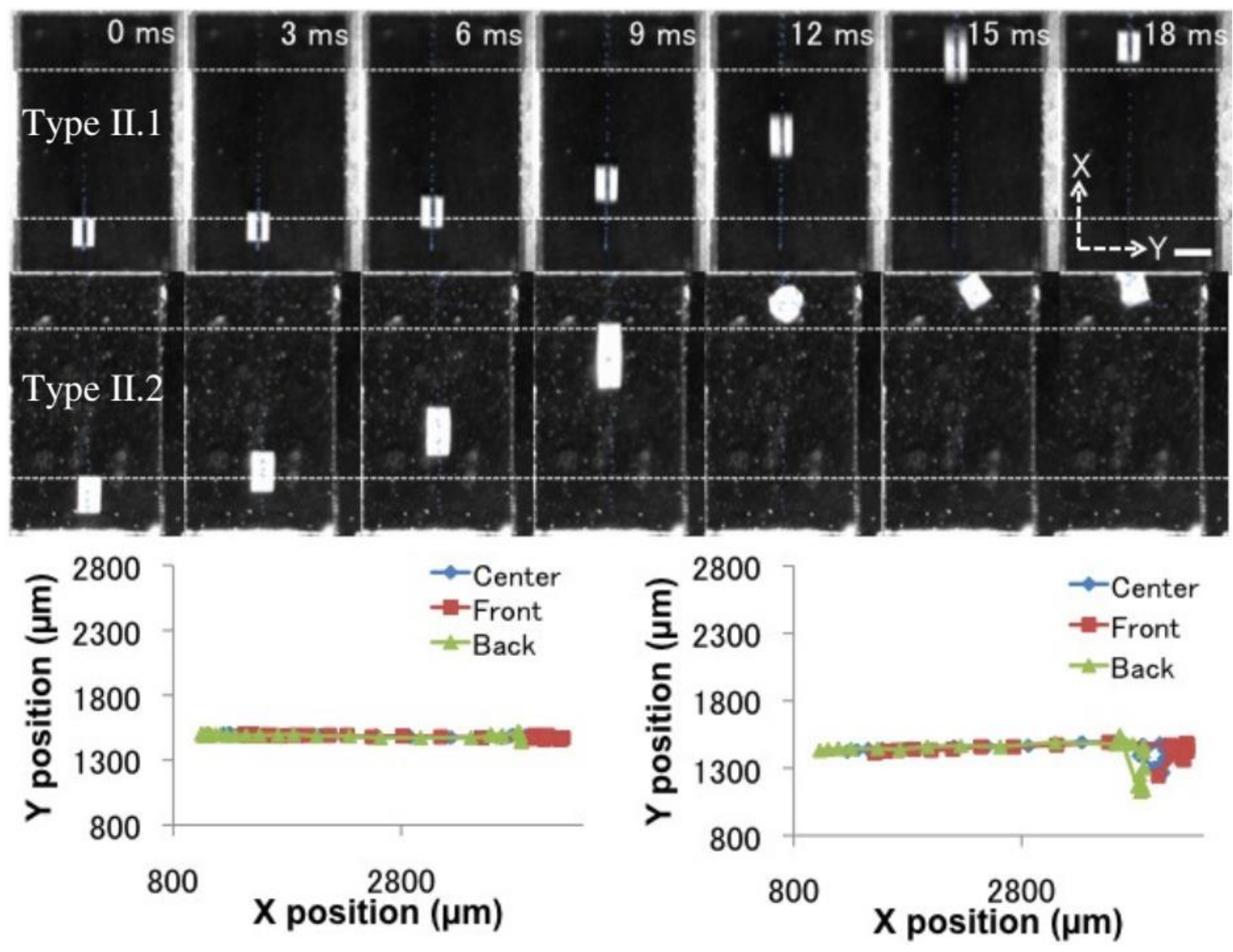




\section{WILEY-VCH}

Figure 9. Frames from a video of a MagPieR traversing a surface, and trajectory plots for two different types of MagPieR.

\subsection{Microrobots Operating in 3D in a Fluid by Gradient Pulling}

A magnetic microrobot in a fluid medium can levitate and move in $3 \mathrm{D}$ by the application of external magnetic field gradients, which act to pull the microrobot in desired directions. These microrobots typically operate in a liquid environment, where viscous damping forces act to slow down the motion of the microrobot (as compared to a gaseous environment), making it controllable. Two systems are discussed in this section, the OctoMag and the system developed by Diller et. al. for multiple microrobot control. ${ }^{[52,53]}$ In other sections, magnetic fields and torques can be used to propel microrobots in liquids, with actuation mechanisms inspired from nature.

\subsubsection{OctoMag}

The OctoMag is an electromagnetic system intended to control microrobots for human surgical procedures in liquid environments, such in intraocular therapy and diagnosis. ${ }^{[52]}$ A set of eight electromagnetic coils is designed to surround a human head. These coils provide energy inputs to operate a levitating magnetic microrobot with 5-DOF control in a relatively large $25 \mathrm{~mm}$ diameter workspace, shown in Figure 10.

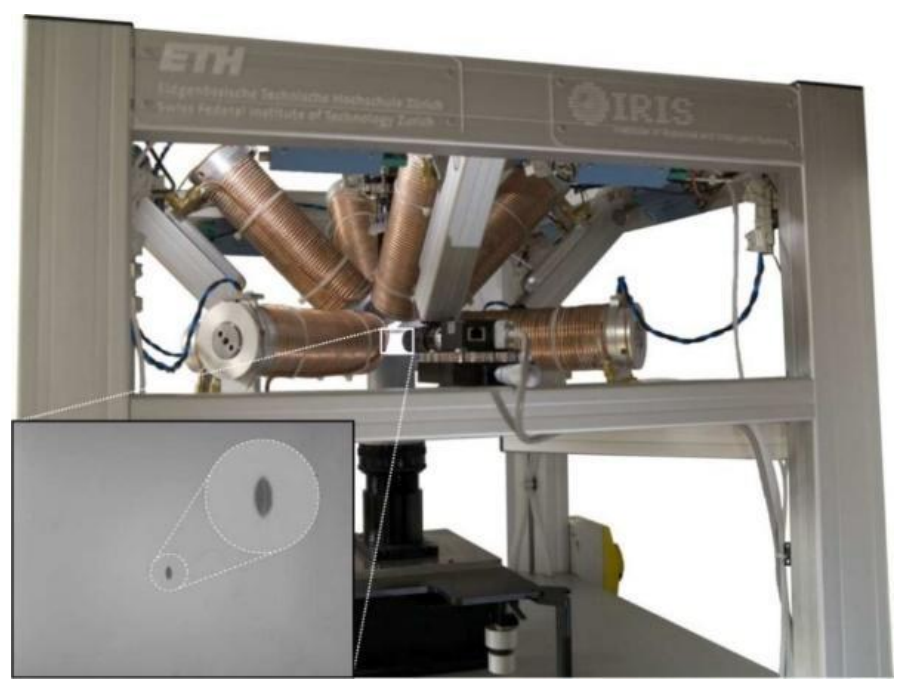




\section{WILEY-VCH}

Figure 10. Experimental apparatus of the Octomag system, showing eight electromagnetic coils surrounding a workspace. Inset shows an optical image of an oval-shaped magnetic microrobot in the workspace.

The microrobot itself can be comprised of a permanent or soft-magnetic material. One type of microrobot is comprised of $\mathrm{Ni}$ or $\mathrm{CoNi}$ and is built into an oval shape (inset in Figure 10, schematically shown in Figure 11), with maximum dimensions varying from 500-2000 $\mu \mathrm{m}$. Another microrobot useful for biopsy is a pair of NdFeB permanent magnet cubes $(800 \mu \mathrm{m}$ cube edge) with a $1.2 \mathrm{~mm}$ hypodermic needle attached (Figure 12d).

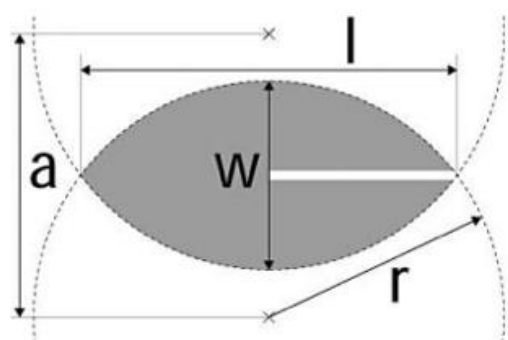

(a)

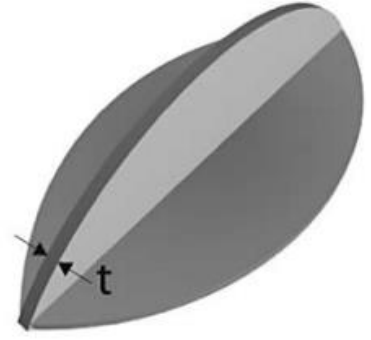

(b)

Figure 11. Schematic of an oval-shaped Ni or CoNi microrobot.

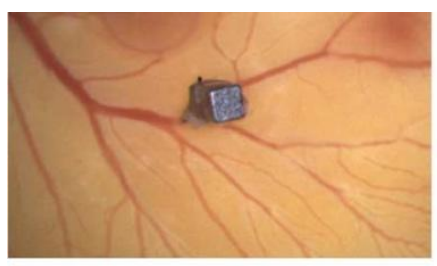

(a)

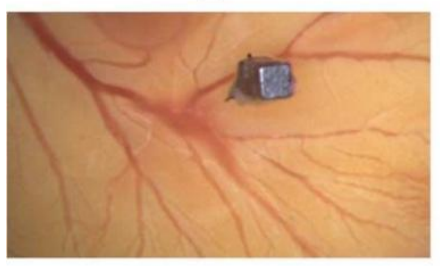

(c)

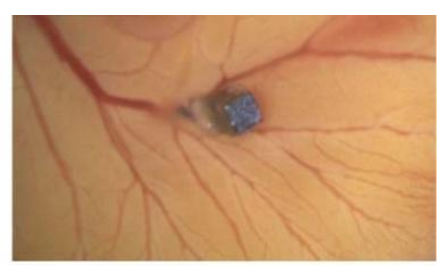

(b)

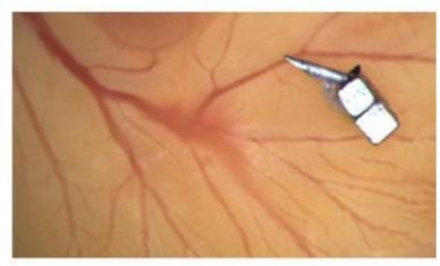

(d)

Figure 12. (a) The Octomag system controlling a NdFeB microrobot with a hypodermic needle tip on a chicken embryo. In (b), the microrobot punctures the embryo, (c) retracts, and (d) microrobot lays on the embryo. 


\section{WILEY-VCH}

Magnetic field gradients are applied to create force, which accelerates the microrobots. A constant magnetic field of $15 \mathrm{mT}$ is applied to orient the microrobot. To resolve the problem of ferromagnetic systems being inherently unstable (Earnshaw's theorem), an active closed-loop visual feedback system is implemented to create stable microrobot position control. The Octomag system can both control position and orientation of the microrobot throughout the workspace using this visual feedback system, and servo the microrobot through arbitrary trajectories (Figure 13).

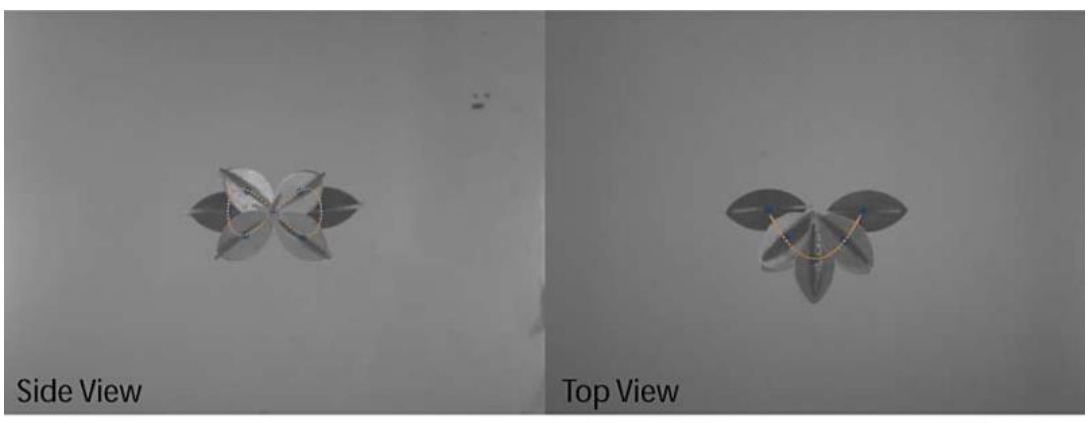

(a)

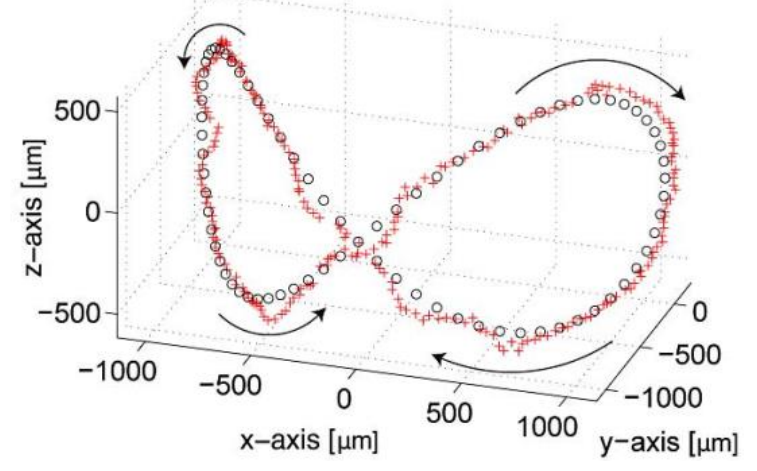

Figure 13. An oval microrobot being served by the Octomag system in a figure-eight trajectory in the workspace.

In addition to motion, the OctoMag can perform surgical tasks. The microrobot in Figure 12 is used to operate on a chicken embryo, where it successfully punctures large blood vessels for biopsy purposes. 


\section{WILEY-VCH}

\subsubsection{Multiple 3D microrobots}

Diller et. al. investigate methods to control multiple 3D microrobots independently, which are levitating in a fluid environment. ${ }^{[53]}$ Eight electromagnetic coils are utilized to provide magnetic fields of up to $8.3 \mathrm{mT}$, and gradients of up to $0.34 \mathrm{~T} / \mathrm{m}$, which orient and apply forces to magnetic microrobots in the workspace. The setup of this system allows for full 6-DOF control of the microrobot, allowing for any microrobot position and orientation to be achieved in the workspace. Microrobots are fabricated to be $400-4000 \mu \mathrm{m}$ in size, can consist of a permanent or soft magnetic material, and can have polymer additions, such as "wings" (Figure 14). An air cavity can be integrated to create buoyant microrobots. 


\section{WILEY-VCH}
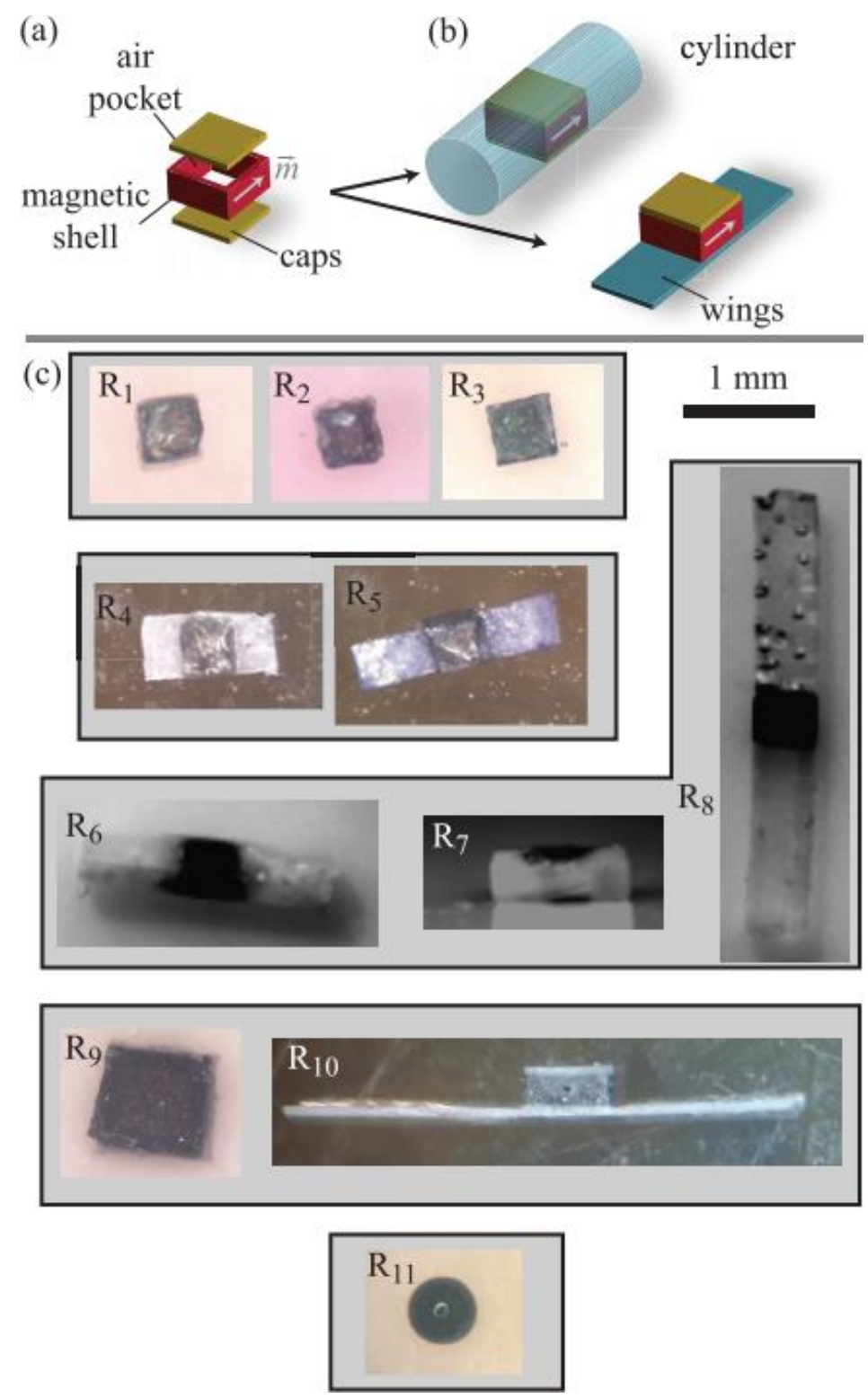

Figure 14. (a)-(b) Schematics of a microrobot agent with an air pocket for buoyancy, and wings for increased fluid drag coefficient. (c) Optical micro-graphs of various speciesof microrobots.

To enable the selection of individual microrobots in the presence of approximately uniform magnetic fields inside the workspace, heterogenous microrobots are utilized, with different geometries and properties. Under an externally applied magnetic field, which is used to orient or rotate a microrobot, microrobots with differing magnetizations or geometries with varying fluid drag coefficients will rotate in the fluid at different angular rates; this incurs a phase lag in motion. Thus, during these rotation events, heterogenous microrobots can instantaneously have different 


\section{WILEY-VCH}

orientations. When a magnetic field gradient is applied, which causes acceleration, microrobots with different instantaneous orientations will accelerate towards different headings.
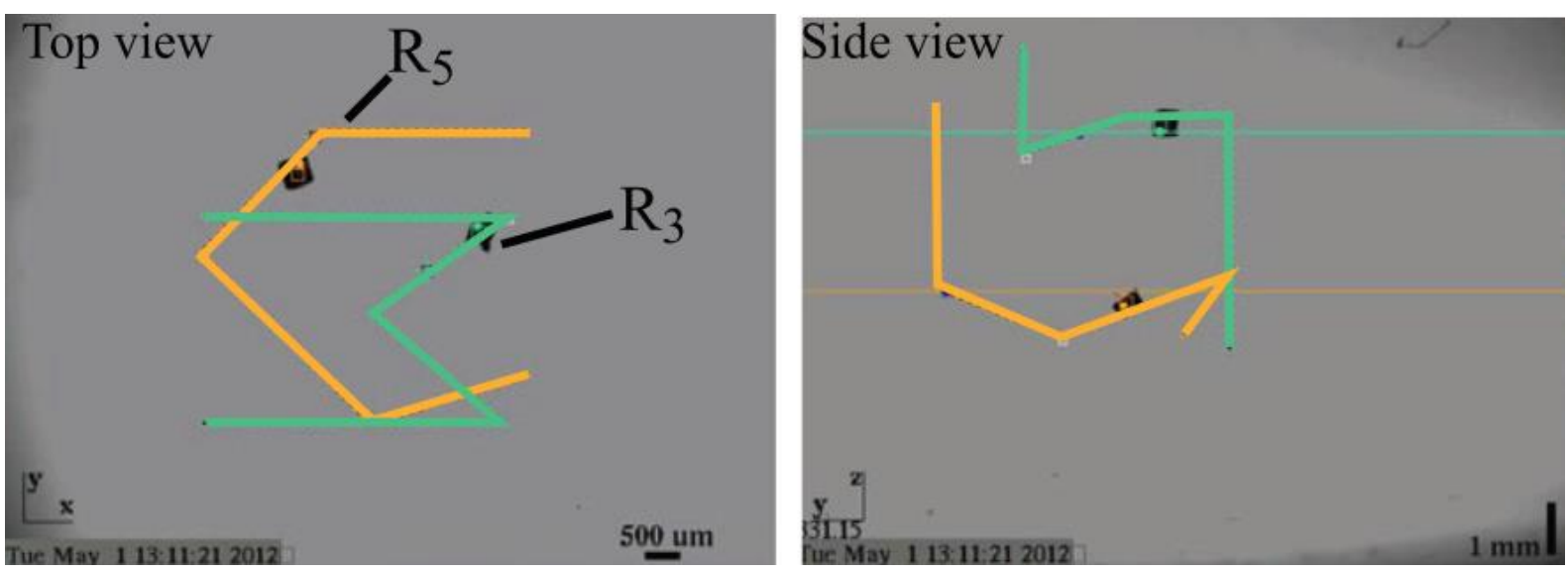

Figure 15. Two microrobots being independently positioned in 3D, following a path.

Using a two-camera vision feedback system, combined with a feedback controller that comprehends the microrobot's phase lag due to fluid interactions, two microrobots were independently and simultaneously positioned in 3D, while following a path (see Figure 15). The ability to move three microrobots in different directions was also demonstrated, see Figure 16.
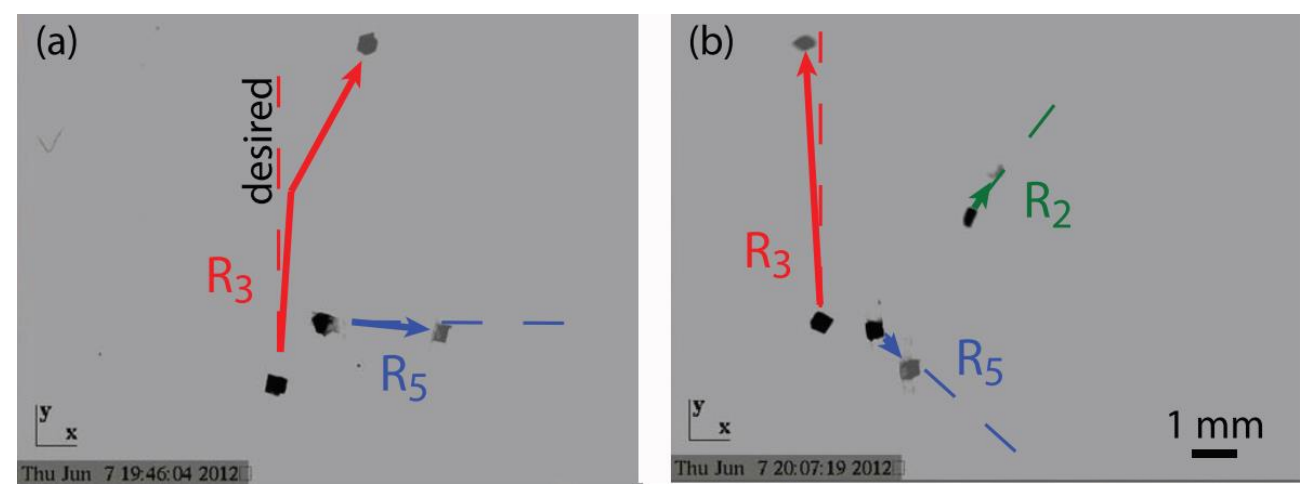

Figure 16. (a) Two microrobots moving along perpendicular headings, (b) three microrobots moving with different headings, in 3D.

\section{Tumbling and Rolling Magnetic Microrobots [Cappelleri]}

Tumbling and rolling magnetic microrobots locomote by rotating over solid surfaces under an applied magnetic torque. At the microscale, the inertia produced from rotational motion alone is 


\section{WILEY-VCH}

not sufficient to produce net displacement in low Reynolds number fluid environments. A solid surface is necessary to create dissimilar boundary conditions between different ends of the untethered microrobot, allowing for forward propulsion.

Compared to direct pulling using magnetic field gradients, tumbling and rotating locomotion has several advantages. At smaller scales, magnetic torque demonstrates higher efficiency than magnetic force under the same external magnetic field. ${ }^{[54]}$ Magnetic force scales with the volume of magnetic material (i.e. $L^{3}$ ) while the equivalent force from magnetic torque scales with $L^{2}{ }^{[55]}$ As a result, rotation induced through magnetic torque is generally preferred at the microscale over force-based pulling. Additionally, rolling friction is less than sliding friction at all size scales. The stiction resisting pulling locomotion over solid surfaces is much higher than the stiction resisting tumbling and rolling locomotion. This behavior reduces the actuation effort required for the latter method and also avoids the sudden acceleration that occurs when large stiction forces are broken, eliminating the need for additional damping effects. Finally, tumbling and rolling magnetic microrobots are capable of traversing over 3D surfaces with inclines, valleys, and other complex surface features in dry conditions while gradient-pulled magnetic microrobots are limited to operation within fluid environments. These qualities make tumbling and rolling magnetic microrobots well-posed for applications with low-strength magnetic fields over unpredictable, complex terrains.

Despite differences in definition, the terms 'tumbling' and 'rolling' are sometimes used interchangeably in microrobot literature, both indicating a combination of rotational and translational motion. In general, rolling refers to the rotating motion of a spherical, ovoid, or cylindrical object spinning about an axis that stays fixed relative to the object's body. Tumbling refers to the cyclical rotation produced from repeatedly falling end-over-end, typically associated with rod-like objects with non-circular cross-sections. In this case, the rotational axis will shift 


\section{WILEY-VCH}

relative to the object's body during the motion cycle. Tumbling can also refer to gaits where an unstable state, similar to falling motion of an inverted pendulum, occurs during the motion cycle. ${ }^{[56]}$ For magnetic microrobots, similar actuation methods are used for both tumbling and rolling locomotion, with robot geometry and the location/orientation of the axis of rotation being the primary differences.

Tumbling and rolling microrobots are typically actuated by the projection of a rotating magnetic field on the robot, though other forms of field modulation can be used as well. Electromagnetic coils are often used as the field source due to their lack of moving parts and ability to create spatially uniform fields, but rotating permanent magnets can also be used. These magnets are more straightforward to implement, require no electrical power to maintain the field, and are well suited for sustaining large magnetic fields. However, the magnetic field of a permanent magnet cannot be turned off like an electromagnetic coil and field manipulation is only possible through physical movement of the magnet. This limitation can lead to stray field gradients that pull on the microrobot as the permanent magnet is rotating, causing undesirable sliding motion.

Rolling locomotion using a rotating permanent magnet was demonstrated by Jiang et al. using a spherical magnetic microrobot. ${ }^{[57]}$ The microrobot consisted of a $440 \mu \mathrm{m}$ diameter UV adhesive ball encapsulating a $30 \mu \mathrm{m}$ diameter iron wire. Cohesive forces produced the spherical geometry after UV adhesive was dripped directly onto a suspended iron wire. The resulting microrobot was shown to be capable of moving in air, water, and silicone oil over flat and bumpy surfaces at speeds of up to $13.2 \mathrm{~mm} \mathrm{~s}^{-1}$. It could also be manipulated to move on pre-defined two-dimensional trajectories.

Individual magnetic wires without encapsulating material are also capable of tumbling locomotion within liquid environments near solid surfaces. Rather than direct surface contact, hydrodynamic interactions occur between the wires and nearby walls when the wires are rotated. These 


\section{WILEY-VCH}

interactions result in dissimilar boundary conditions between the two ends of the wire, yielding non-reciprocal motion and allowing for forward propulsion in low Reynolds number environments. Zhang et al. showed Ni nanowires ranging from 10 to $30 \mu \mathrm{m}$ in length tumbling over flat surfaces and climbing vertical microchannel walls. ${ }^{[58]}$ The wires could penetrate a $6 \mu \mathrm{m}$ polystyrene microbead onto one end and transport it to a target location in a controlled manner. Compared to direct pushing methods using catalytic nanowire motors, the tumbling nanowires experienced significantly less drag resistance from the cargo due to its non-contact nature with the underlying substrate. ${ }^{[59,60]}$ Various step-out field rotational frequencies were observed for different lengths of wire, where the available magnetic torque was insufficient for counteracting fluid drag and keeping the wire's rotation synchronized with the rotating magnetic field. This limitation could be circumvented by increasing the field strength of the applied external field.

Mair et al. loaded alginate capsules with aligned ferromagnetic nanorods to create a microrobotic platform (MANiACs) for delivering biomedical payloads (Figure 17a). ${ }^{[61]}$ The high magnetization of the fixed dipole nanorods allowed the capsules to be rotated with small magnetic fields using relatively low loading volume fractions. The capsules demonstrated guided tumbling locomotion on glass surfaces, biological tissue surfaces (rat intestine), and inclined surfaces (up to $15^{\circ}$ ) over centimeter-scale distances. As a group, they were able to manipulate other exterior objects, pushing a T-shaped microstructure four times their individual size across a flat surface in liquid. When loaded with a model small molecule in a controlled release study, the alginate capsules exhibited combined tumbling translation and payload release over a one-hour period. Pausing the tumbling locomotion led to increased molecular concentrations at given locations and allowed for location-specific payload delivery.

Self-assembling colloidal rotors can tumble along surfaces in the presence of a rotating magnetic field (Figure 17b) ${ }^{[62]}$ Superparamagnetic beads were observed to assemble together in chains when 


\section{WILEY-VCH}

an external field was applied. The chains of beads behaved like elongated magnetic magnets and were held together solely with magnetic forces, allowing for their reversible assembly and disassembly. The length of the chains could be dynamically adjusted through the disassembly and assembly process to cater it towards a specific application or movement speed. As field rotational frequencies increased, fluid-induced drag forces overcame the cohesive forces between the beads and chain breakup occurred, resulting in reduced translational velocity. A simulation model was developed to accurately predict when the critical chain breakup frequency would occur. When collected in large numbers, groups of superparamagnetic bead chains were able to support and precisely move vesicles several times larger than the length of an individual chain.

A variety of additional geometries and substances have been demonstrated to tumble in liquid environments as well, including iron-containing multiwalled carbon nanotubes, red blood cells with attached magnetic particles, and DNA-linked anisotropic doublets of paramagnetic colloidal particles. ${ }^{[63,64]}$ In liquid environments, instabilities during the tumbling gait are dampened by fluid drag and buoyancy forces. Many rod-like microrobots with geometry not optimized for tumbling locomotion can tumble under the presence of a rotating magnetic field. For example, helical magnetic micro-swimmers were observed to tumble after hippocampal neural stem cells were cultured on their surface. ${ }^{[37]}$ When the cells were removed, the micro-swimmers rotated about their long axis as intended in a corkscrew motion.

Untethered magnetic micro-manipulators rotating in viscous fluid induce local rotational fluid flows that can move micro-objects in the flow region without direct contact with the manipulator. ${ }^{[65]}$ A transversely magnetized rolling magnetic microrobot (RodBot) exploited this behavior to perform non-contact manipulation and transportation of micro-objects in liquids (Figure 17c). ${ }^{[66,67]}$ The microrobot, a $50 \times 60 \times 300 \mu \mathrm{m}$ polymeric block with internal cobalt-nickel posts, rolls continuously about its long axis to generate a fluid vortex above it while simultaneously 


\section{WILEY-VCH}

translating forward. The vortex attracts and traps micro-objects without direct contact, offering coarse motion control of delicate, tiny objects ranging from a few microns to several hundred microns in size. Forces from the vortex range from a few nanoNewtons to tens of nanoNewtons and are spread over the surface of the object. Rising fluid flow generated in front of the microrobot from the rolling motion helps with trapping objects within the vortex. Using real-time visual tracking of the microrobot and surrounding obstacles, a holistic system was developed to automate the motion of the microrobot for protein crystal harvesting. ${ }^{[68-70]}$ The microrobot was also capable of directly pushing $200 \times 350 \mu \mathrm{m}$ SU-8 micro-objects into a densely packed formation within a narrow channel.

While tumbling magnetic microrobots are typically actuated with a rotating magnetic field, Wuming et al. developed a tumbling microrobot actuated using an alternating magnetic field. ${ }^{[71,72]}$ Here, a composite magnetic structure of a dumbbell shape was designed. The two 'bell' ends of the structure each consisted of permanent magnets polarized in opposite directions, and a nonmagnetic bridge piece connected the ends. An electromagnetic coil system alternating between horizontal and vertical magnetic fields cycled the microrobot through different orientations, using forward momentum to produce a tumbling motion. The opposing magnetic polarities on each of the microrobot's ends ensured the resulting tumbling rotation maintained consistent directionality. Steering was achieved by changing the orientation of the projected magnetic field in the horizontal plane. The microrobot was able to move in dry and fluid environments on various 3D surfaces, including a tissue sample in saline, and capable of directly pushing SU-8 micro-objects using field gradients in liquid environments.

Tumbling locomotion using rotating magnetic fields exhibits similar performance over arbitrary surfaces in dry air conditions. Hou et al. demonstrated a rectangular stainless steel microrobot tumbling over an acrylic plate and over the surface of a coin. ${ }^{[73]}$ The microrobots were shown to 


\section{WILEY-VCH}

generate large forces relative to their size, capable of lifting small pieces of paper approximately $7.8 \mathrm{mg}$ in mass. Bi et al. further characterized tumbling motion using rectangular, polymeric NdFeb microrobots ( $\mu \mathrm{TUM}$ ), which showed improved magnetic response and configurable magnetic polarization (Figure 17d). ${ }^{[74]}$ Photolithography using an SU-8/NdFeB microparticle slurry allowed for arbitrary 2D geometries on the magnetic ends of the microrobot. Various end geometries, including a triangular point and spiked corners, were tested and shown to be ineffectual for improving microscale mobility. Maximum incline climbing angles were measured to be $45^{\circ}$ and $60^{\circ}$ in dry air and liquid environments, respectively. The tumbling microrobot was also able to traverse over complex, patterned surfaces with protruding features and trenches smaller than the robot's body length. To better predict the impact of different design and environmental parameters, a novel simulation model was developed - capable of simulating the intermittent and non-point contact that occurs during tumbling locomotion. ${ }^{[75]}$ The microrobot was able to locomote in vivo, inside a murine colon, while being observed through visual occlusions using ultrasound imaging, and could be functionalized with a drug payload coating. ${ }^{[76]}$

Soft magnetic materials can lead to microrobots capable of reconfiguration between tumbling and rolling locomotion. Huang et al. developed shape shifting soft microrobots made from self-folding hydrogel bilayer structures that morphed in response to temperature changes (Figure 17e). ${ }^{[77]}$ Ondemand modulation of individual microrobot mobility was demonstrated by morphing their shape using selective near infrared light (NIR) exposure. The magnetized hydrogel bilayer starts off as a planar sheet, folding into a tube shape when temperature decreases below a critical threshold ( 40 $\left.{ }^{\circ} \mathrm{C}\right)$. When temperature increases above this threshold, the sheet refolds in the opposite direction around the perpendicular axis. Between the two shape configurations, the orientation of the folded tube changes with respect to the polarity of embedded magnetic particles and the microrobot can alternate between tumbling and rolling locomotion. Using NIR lasers, the tube radius and 


\section{WILEY-VCH}

configuration of individual microrobots could be selectively controlled within a group. Tuning the tube radius directly affects the translation velocity of the microrobot, leading to differential motion control within a global magnetic field. Hu et al. also demonstrated microrobots capable of transiting between rolling and tumbling locomotion, among a variety of other locomotive modes. ${ }^{[78]}$ These magneto-elastic microrobots used interactions between their non-uniform magnetization profiles and changes in the magnetic strength/orientation of the external field to curl and undulate into various shapes and gaits. 


\section{WILEY-VCH}

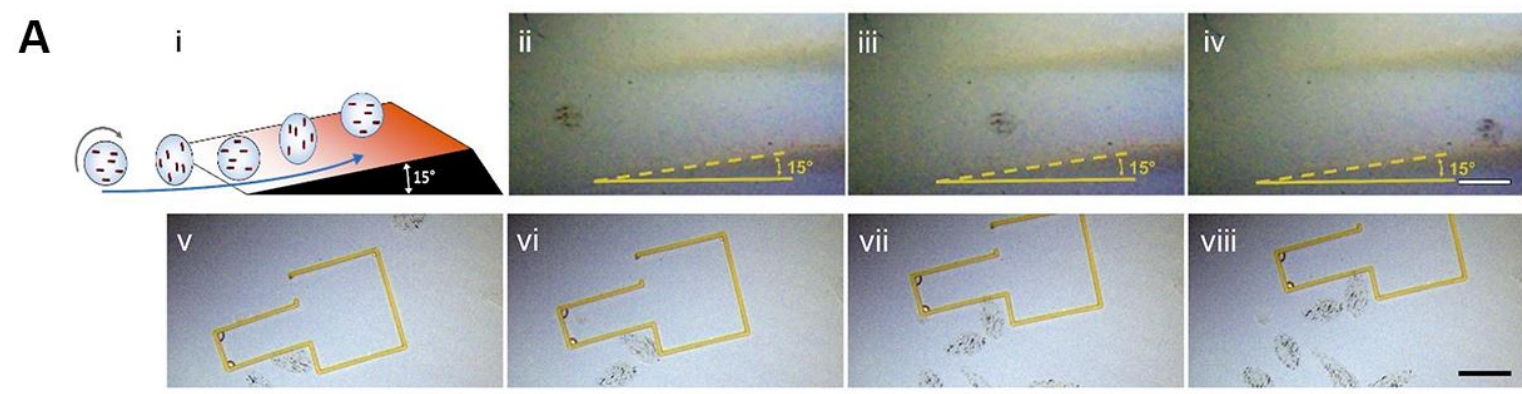

B i

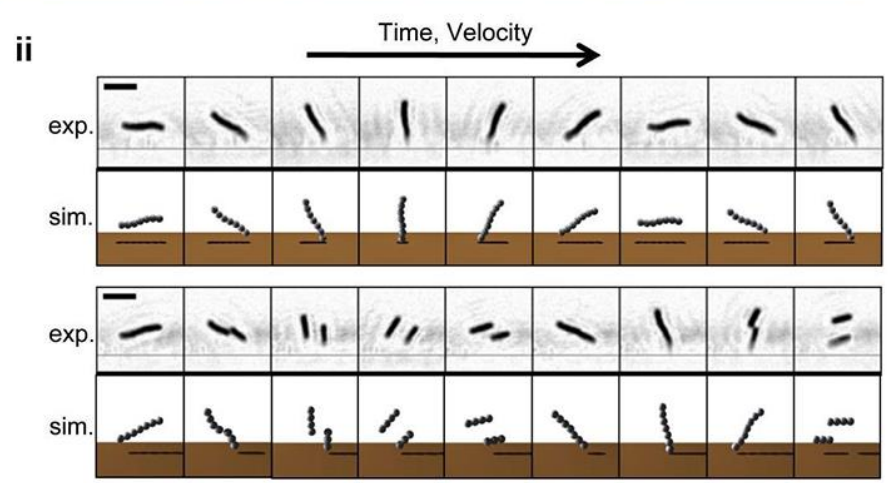

C
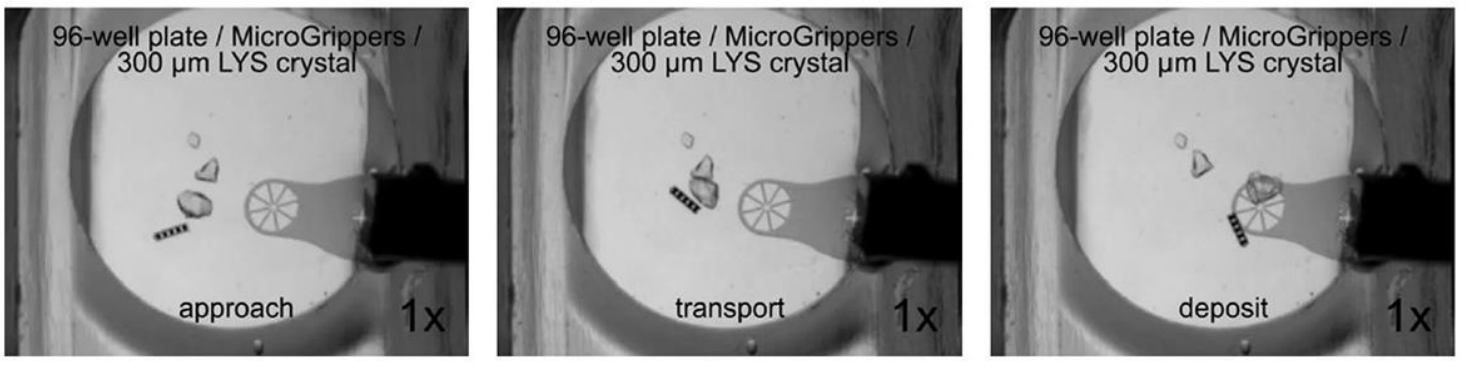

D
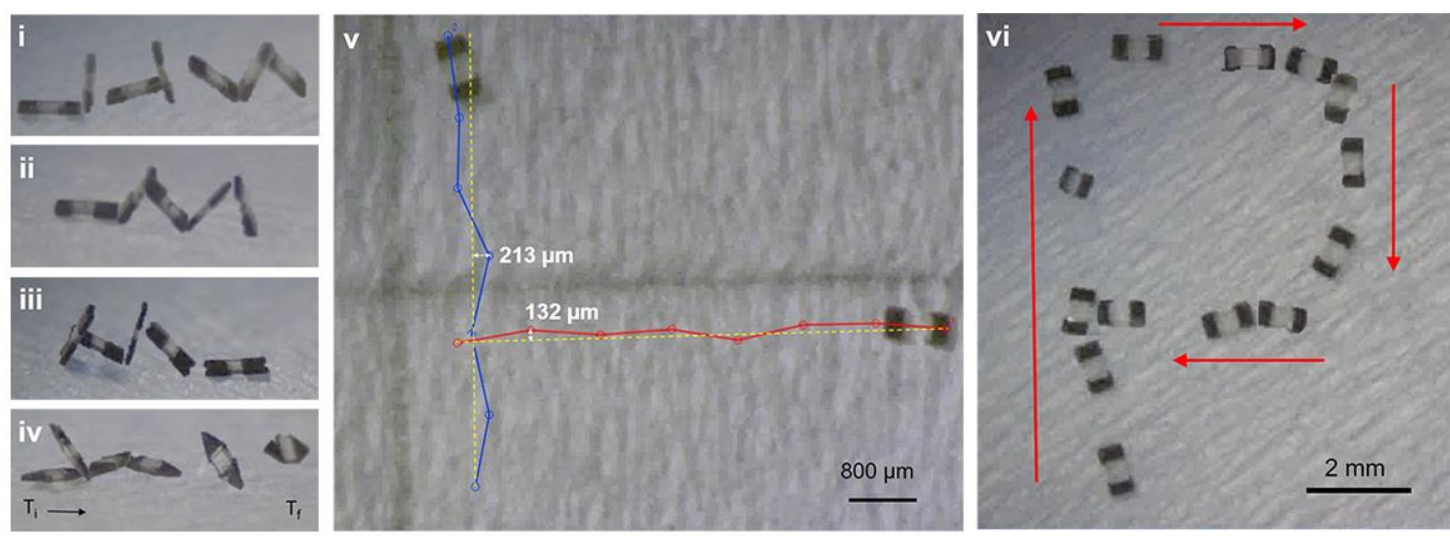

E
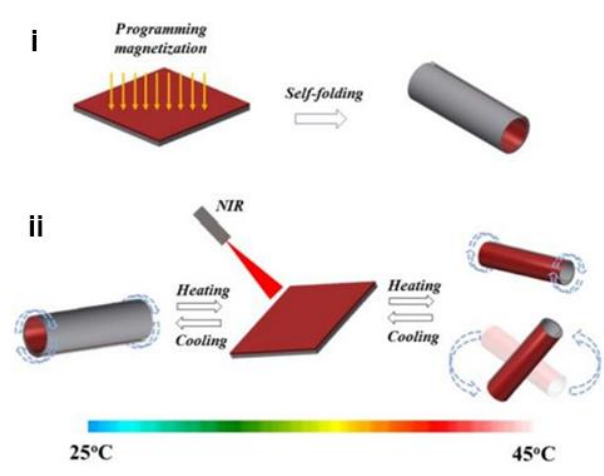

iii

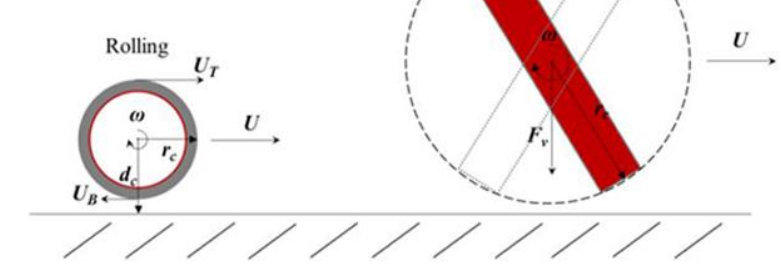


Figure 17. Tumbling and rolling magnetic microrobots. A) Rotating magnetic alginate capsules (MANiACs) are able to climb inclines and push photoresist structures several times their individual size. i) Schematic depicting MANiACs rotation and incline climbing. ii-iv) An alginate capsule climbing a $15^{\circ}$ incline. Scale bar is $1 \mathrm{~mm}$. v-viii) MANiACs are tumbling next to the corner of a hollow photoresist structure, resulting in successful manipulation by pushing the structure on a smooth glass surface. Reproduced with permission. ${ }^{[58]}$ Copyright 2019, MDPI AG. B) Self-assembled colloidal rotors exhibit tumbling motion near solid surfaces under a rotating magnetic field. i) The geometry of an individual rotor. Each chain is composed of superparamagnetic beads that move according to the dynamics of the magnetic field $B$ that induces a magnetic moment $m$ on each bead. ii) Rotation along the $x-z$ plane at a frequency $v$. Top: The aggregate moves along the surface in both experiment (frames taken $16 \mathrm{~ms}$ apart in a $5 \mathrm{~Hz}$ rotating field, scale bar is $5 \mu \mathrm{m}$ ) and simulation upon confinement at the surface. Bottom: When the field rotation is raised to $7 \mathrm{~Hz}$, the rotors fragment periodically. Reproduced with permission. ${ }^{\text {[62] }}$ Copyright 2010, National Academy of Sciences. C) Microscopic view of RodBot crystal manipulation with a fluidic vortex. The RodBot lifts, transports, and deposits a lysozyme crystal towards an extraction-tool without physical contact. The RodBot and the crystal both have a maximum extent of $300 \mu \mathrm{m}$ and are submerged in a 20\%PEG3350 solution. Reproduced with permission. ${ }^{[67]}$ Copyright 2015, IEEE. D) Motion lapse images of NdFeB tumbling microrobots ( $\mu$ TUM). i-iv) Sideview tumbling gaits of i) rounded rectangular, ii) asymmetric rounded rectangular, iii) rounded protruding corner, and iv) triangular end geometry variations. v) $\mu \mathrm{TUM}$ tumbling trajectories (blue/red) relative to an ideal $5 \mathrm{~mm}$-long straight-line trajectory (yellow); the maximum trajectory drift for each is reported. vi) Rectangular $\mu$ TUM traversing in a P-shaped trajectory using open-loop control. Reproduced with permission. ${ }^{[74]}$ Copyright 2018, MDPI AG. E) Diagrams of shape shifting microrobots generated from magnetized hydrogel bilayer structures. i) Programmable magnetization and folding of hydrogel bilayers to generate magnetically controllable microrobots. ii) Shape switching concept for soft microrobots by increasing temperature using near infrared light exposure. iii) Free body diagram of the microrobot rolling and tumbling on a surface. Reproduced with permission. ${ }^{[77]}$ Copyright 2016, IEEE.

\section{Magnetotactic Bacteria [Damien]}

Magnetotactic bacteria (MTB) are aquatic microorganisms that are capable of biomineralizing nanometric magnetic particles (iron oxide or iron sulphide) supposedly to navigate along the earth's magnetic field lines ${ }^{[79]}$ These magnetic particles are protected by a biocompatible membrane and the whole, combined structure of particle and membrane, are commonly known as magnetosomes. ${ }^{[80,81]}$ The size and shapes of the magnetite nanoparticles are genetically controlled and possess a single magnetic domain. Inside the bacteria, these magnetosomes align forming chains that provide a "compass" or "navigation system" by which the bacteria follow the Earth's magnetic field lines. The MTB compose a large, diverse and phylogenetic group of aquatic 


\section{WILEY-VCH}

bacteria, its motility is powered by its flagella. An overview of different commonly found strain types and their corresponding reported properties is provided in Table 1. Some typical bacteria cellular structures are shown in the TEM images of Figure 18. Even though the collection of MTB from their natural environments may be relatively easy to achieve, the MTB are not easy to isolate or cultivate. Different strains might need different cultivation conditions. ${ }^{[82]}$ A large number of magnetosome-producing microorganisms exist and are classified. ${ }^{[81]}$

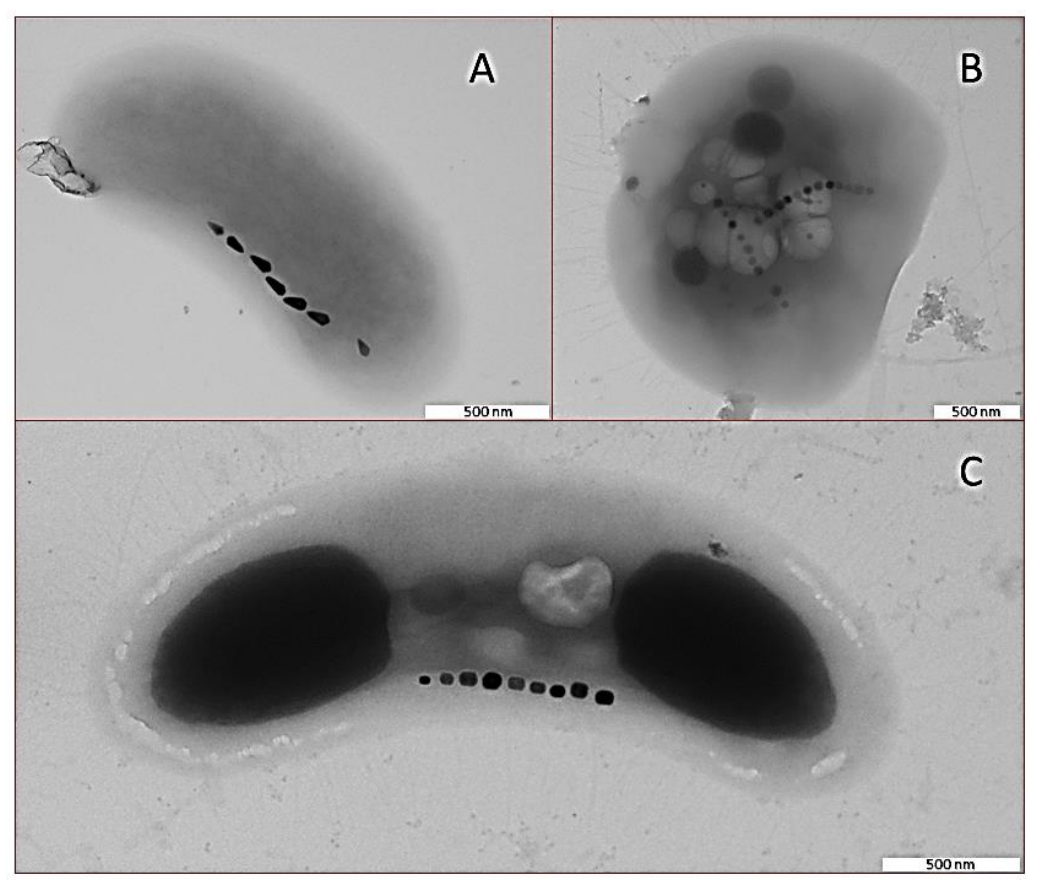

Figure 18. TEM images of environmental magnetotactic bacteria with A) rod, B) coccoid or C) vibrio cell shape. In addition, different shaped magnetosomes are distinguished: A) elongated octahedral, B) cubooctahedral and C) rectangular morphologies- Courtesey of Dr. Christopher T. Lefèvre 
Table 1. Shape, size, motility, magnetosome and magnetoaerotactic properties of several magnetotactic bacteria strains. \# average speed at the anoxic zone * Info provided by courtesy of Dr. C. T Lefèvre.

\begin{tabular}{|c|c|c|c|c|c|c|}
\hline $\begin{array}{l}\text { Magnetotactic } \\
\text { Bacteria strain }\end{array}$ & Cell shape & Size $[\mu \mathrm{m}]$ & $\begin{array}{l}\text { Average } \\
\text { speed }^{\#}\left[\mu \mathrm{m} \mathrm{s}^{-1}\right]\end{array}$ & $\begin{array}{l}\text { Magnetsome } \\
<\text { crystal } \\
\text { length }>(\mathrm{nm})\end{array}$ & $\begin{array}{l}\text { Magnetosome } \\
\text { Shape }\end{array}$ & Flagela \\
\hline $\begin{array}{l}\text { Magnetococcus } \\
\text { marinus (MC-1) }\end{array}$ & Cocci & $1-2$ & $119 \pm 13.6$ & $30-110$ & $\begin{array}{l}\text { Elongated } \\
\text { octahedral }\end{array}$ & $2 \times 7$ \\
\hline $\begin{array}{l}\text { Magnetospirillum } \\
\text { gryphiswaldense } \\
\text { (MSR-1) }\end{array}$ & Spirillum & $3-4$ & $23.3 \pm 2.9$ & 33 & Cuboctahedral & 2 \\
\hline $\begin{array}{l}\text { Magnetospirillum } \\
\text { magneticum (AMB- } \\
\text { 1) }\end{array}$ & Spirillum & $3-4$ & $49 \pm 20$ & 45 & Cuboctahedral & 2 \\
\hline $\begin{array}{l}\text { Magnetovibrio } \\
\text { blakemori } \\
\text { (MV-1) }\end{array}$ & Vibrio & $1-3$ & $8.5 \pm 2.3$ & 60 & $\begin{array}{l}\text { Elongated } \\
\text { octahedral }\end{array}$ & 1 \\
\hline $\begin{array}{l}\text { Magnetospirillum } \\
\text { magnetotacticum } \\
\text { (MS-1) }\end{array}$ & Spirillum & $5.2 \pm 0.5$ & 44 & $30 \pm 8$ & Cuboctahedral & 2 \\
\hline UT-4 & Spirillum & $2-4$ & $25.9 \pm 1.4$ & $10-50$ & Cuboctahedral & 2 \\
\hline PR-1 & Spirillum & 1.6 & $50.5 \pm 5.3$ & $31-57$ & $\begin{array}{l}\text { Elongated } \\
\text { octahedral }\end{array}$ & 2 \\
\hline PR-2 & Vibrio & no info & $23.7 \pm 3.6$ & Around 50* & $\begin{array}{l}\text { Elongated } \\
\text { octahedral }\end{array}$ & 1 \\
\hline LM-1 & Vibrio & $3-5$ & $20.7 \pm 1.5$ & $20-60$ & $\begin{array}{l}\text { Elongated } \\
\text { octahedral }\end{array}$ & 1 \\
\hline SS-5 & Rod & $4-5$ & $32.1 \pm 2.2$ & 86 & $\begin{array}{l}\text { Elongated } \\
\text { octahedral }\end{array}$ & 1 \\
\hline $\begin{array}{l}\text { Magnetospira } \\
\text { thiophila (MMS-1) }\end{array}$ & Spirillum & 0.5 & $49.7 \pm 10.6$ & $22-85$ & $\begin{array}{l}\text { Elongated } \\
\text { octahedral }\end{array}$ & 2 \\
\hline SS-1 & Coci & no info & $111.6 \pm 21$ & No info & $\begin{array}{l}\text { Elongated } \\
\text { octahedral }\end{array}$ & $2 \times 7$ \\
\hline PR3 & Cocci & $\sim \mathrm{MC}-1 *$ & $109.9 \pm 13.2$ & Around 50* & $\begin{array}{l}\text { Elongated } \\
\text { octahedral }\end{array}$ & $2 \times 7$ \\
\hline
\end{tabular}




\section{WILEY-VCH}

Salvatore Bellini in 1963 found aquatic microorganisms that always moved in the same direction under his microscope. ${ }^{[94]}$ He observed that the phenomena persisted no matter how much he rotated the microscope support. Tests were done by putting on a slide with coverslips and changing its local environment (amount of water and extraction point). The bacteria always were attracted in the same matter. A wooden microscope arm and support was prepared but still the same occurred. It was concluded that the microorganism headed towards the geomagnetic north. To verify this hypothesis, he decided to observe and see if they behave the same outside their biological environment. For this, he dispensed two hanging drop of water next to each other, one containing the observed microorganism at the south and another sterilized drop of water at the north and observed the migration of microorganism going from south to north. Later, he tested applying external magnetic fields and observed how they increased speed upon increasing field strengths. His conclusion states that some anaerobic bacteria always head towards the magnetic North Pole and proposed the denomination "Magnetosensitive Bacteria". He also remarked that there must exist a biomagnetic "compass" in their body.

Independently, Richard Blakemore found similar microorganisms as Salvatore Bellini in 1975. ${ }^{\text {95] }}$ In his findings, he studied the bacterial tactic response towards external applied magnetic fields and used the term magnetotaxis to describe the observed phenomenon. He presents the first TEM images of the bacteria showing its flagella and the iron-rich nanoparticles in the cell body confirming so the hypothesis of Bellini that indeed particles in the cell internal structure act as a compass needle. ${ }^{[96]}$

\subsection{The Biomineralization Process}

A set of proteins control the biomineralization of the magnetosomes defining their size and shape. The exact biomineralization process is unknown, in the sense that not all discovered gene's functionality, i.e. their actual roles in the process, are known. In addition, the existence, nature, and location of possible mineral precursors of magnetite are not clear. Recently it was showed that 


\section{WILEY-VCH}

ferritin-like proteins, that were initially thought to be part of the magnetite biomineralization process involved storing excess iron as an inert iron phase to avoid Fenton Chemistry, are actually not essential for the biomineralization process occurring in MSR-1 but are definitely involved in the resistance to oxidative stress. ${ }^{[97]}$ It is known that the genes encoding magnetosome production are clustered within an about $130 \mathrm{~kb}$-sized genomic region called the Magnetotactic or Magnetosome Island. ${ }^{[98]}$ Among different species, a common group of around 30 genes is identified (referred to as mam and mms genes) and is not present in non-magnetotactic bacteria thus expected to play an important role in the formation of the magnetsomes. The magnetic material needed for the formation of the magnetosomes is generally iron and taken up from the growth medium (or from the environment). A cytoskeletal structure composed of the MamK protein, which is a relative of the eukaryotic cytoskeletal protein actin; together with MamJ attaches the magnetosomes in a strain like structure. ${ }^{[99,100]}$ Recently, the MamY protein was associated to the magnetosome alignment strategy. ${ }^{[101]}$

More detailed information, on the genes involved in the biomineralization of magnetotactic bacteria, can be found e.g. in ${ }^{[102,103]}$

\subsection{The Taxis}

From a biological context, the steering of an entity to provide directed motion by means of an external signal is called taxis. ${ }^{[104]}$ When the motion pattern is altered due to internal energetic condition it is called energy taxis. ${ }^{[105]}$ The external signal can differ of nature being generally chemical (chemotaxis), light (phototaxis), or other like gravity (gravitaxis), magnetic (magnetotaxis), etc. In the specific case of the MTB, it uses a magnetically assisted aerotaxis (magneto-aerotaxis) allowing the MTB to locate and maintain an optimal position in vertical chemical concentration gradients (oxygen) thus minimizing its search area to a single dimension. ${ }^{[106]}$ MTB may be found in sediments or chemically stratified water columns at the oxic- 


\section{WILEY-VCH}

anoxic interface or in the anoxic regions. It is important to understand the MTB taxis as we could benefit or be inspired by its mechanism for application purposes.

\subsection{The Oxic-Anoxic Interface}

A microcapillary assay is typically used in the lab to simulate the oxygen gradient encountered in the environment. ${ }^{[107,108]}$ For this method a suspension of bacteria is loaded into a microcapillary, which is sealed at one end using a petroleum jelly plug and open at the other end. Directly a gradient forms by either the consumption of oxygen by the bacteria or due to the presence of the reducer in the plug. A magnetic field orientated antiparallel to the oxygen gradient is then applied. Migration towards the preferred oxygen concentration in the capillary and the orientation of the magnetic field, promotes the initially homogeneously dispersed suspension of bacteria to organize into a densely populated band denominated as the aerotactic band. The insertion of oxygen microprobe into the capillary or a fluorescent dye sensitive to oxygen detects the actual oxygen gradient present in the capillary ${ }^{[109]}$

\subsection{The Molecular Motor: Flagella}

One has to bear in mind that for MTB, the magnetic field provides the directionality. In a homogenous magnetic field, the bacteria will align, as would a compass needle do, but it needs motility in order to propel. Instead of the typical run-and-tumble known for E. coli (REF), a run and reverse is typically observed for MTB. ${ }^{[10]}$ This is, without any U-turn of the cell body, the bacteria inverse their propulsion direction while simultaneously maintaining the direction of their magnetic moment. An overview of flagella apparatus from a set of typical bacteria species is provided in table 1 . The average observed speed can partly be correlated to the different types of flagella they possess. For example, the two sheath of seven flagella that MC-1, PR-3 and SS-1 possess provide the fastest motion compared to the single and biflagellate bacteria. Detailed description on flagella and MTB is available ${ }^{[106]}$ as specific studies for the MC-1 strain. ${ }^{[11]}$ 


\section{WILEY-VCH}

\subsection{Different Sensing Mechanism}

Each MTB species exhibit a different sensing mechanism. The magnetotactic behaviours are described by either one or a combination of dipolar, axial and unipolar mechanism. ${ }^{[83]}$

In the previously described microcapillary assay, dipolar magnetotactic bacteria produce a microaerotactic band and by reversing the magnetic field, the cells swim persistently move away from the initial band. The swimming mode of polar magnetotactic bacteria is a two state sensory mechanism, the activation of either state is determined by the concentration of dissolved oxygen sensed by bacteria and not by the gradient. The direction toward where the bacteria swim is determined if they sense a concentration above or below a given threshold. Above this threshold, north-seeking dipolar bacteria will swim towards the North Pole and below this threshold, they will swim towards the South Pole. ${ }^{[83]}$

In some population of bacteria, such as e.g. MS-1 and MSR-1, no apparent distinction between north- and south-seeking bacteria arises. They move back and forth without any unidirectional motion. This behavior corresponds to axial magnetotaxis, and is only found in pure cultures and not within environmental isolates. In the microcapillary assay, they are not dispersed upon changing the direction of the magnetic field. ${ }^{[83]}$

The unipolar magnetotactic behavior can only be distinguished in the microcapillary assay when the magnetic field is reversed. The microaerotactic band formed by unipolar magnetotactic bacteria swim as a single population either persistently towards north (MV-1, SS-5, PR-1) or south (RS1). ${ }^{[83]}$ Those swimming north can upon field reversal sense the dissolved oxygen concentration. When present at the region with high levels of dissolved oxygen (oxic side of the band), they sense and follow the magnetic field, and nevertheless at low oxygen levels (anoxic side of the band) they sense and follow the oxygen gradient.

MTB have been shown to be able to overcome tortuous flow fields. Studies of AMB-1 navigation in complex flow environments in a microfluidic chip showed that MTB overcome 2.3-fold higher 


\section{WILEY-VCH}

flow velocities when directed to swim perpendicular to a given flow as compared to upstream, as the latter orientation induces higher drag. Magnetotaxis enables MTB to overcome counterdirectional flow at threshold values of drag $(9.5 \mathrm{pN})$ and flow velocity $\left(550 \mu \mathrm{m} \mathrm{s}^{-1}\right) .{ }^{[12]} \mathrm{In}$ addition, MTB migration in porous media is such that MTB circumvent obstacles by repeatedly switching between forward and backward runs. ${ }^{[113]}$ Finally, evidences of a genetic link between aero- and magnetotaxis and magnetotactic polarity were reported. ${ }^{[14]}$

\subsection{Applications of Magnetosomes and Bacteria}

The same way the bacteria uses these magnetosomes to guide themselves, they can interact with any external magnetic field allowing remote control of their navigation, an easy detection, and a means to visualize them. The bacteria are biocompatible, allow easy chemical functionalization and the individual magnetosomes can be harvest and chemically modified. All these properties make both bacteria and magnetosomes potential candidates for a variety of applications.

\subsubsection{Applications of Magnetosomes}

Regarding magnetosomes, their highly desirable single domain magnetic domain, the ability to functionalize their lipid membrane, and their biocompatibility has allowed their usage in several fields such as contrast agents for Magnetic Resonance Imaging (MRI), ${ }^{[115,116]}$ separation of biomolecules, ${ }^{[117-119]}$ cellular homeostasis disorder, ${ }^{[120]}$ hyperthermia therapy, ${ }^{[121,122]}$ photothermia therapy, ${ }^{[123]}$ immunoassays, ${ }^{[124]}$ drug delivery, ${ }^{[125]}$ biosensing (Peroxidase-like activity), ${ }^{[126]}$ and stain removal. ${ }^{[127]}$

\subsubsection{Application of Bacteria}

Living MTB are also used in a variety of application varying from separation of biomolecules, ${ }^{[128]}$ MRI, ${ }^{[129,130]}$ detection of biological entities, ${ }^{[131]}$ hyperthermia therapy, ${ }^{[132]}$ immunoassays, ${ }^{[133]}$ drug delivery, ${ }^{[84]}$ biosensing, ${ }^{[134]}$ pathogen killing, ${ }^{[135,136]}$ domain analysis of soft magnetic 


\section{WILEY-VCH}

materials, ${ }^{[137]}$ waste treatment, ${ }^{[138-142]}$ electromagnetic induction, ${ }^{[143]}$ to model for Human CDFrelated type-II diabetes. ${ }^{[144]}$

However, the aim of the present review is to analyze the magnetotactic bacteria in robotics. Using the bacteria as a micro/nanorobot is easily justified if we analyze the general requirements for a nanorobot designed for nanomedicine. The micro/nanorobot should be able to selectively target a region of interest transporting a given payload. Even more, the micro/nanorobot should be selfpropelled, thus present motility, to reach larger area, be able to sense, and interact with the local environment. They must be detectable in order to track and analyze them. Furthermore, the micro/nanorobot should be programmable or remotely controlled.

This is important not only for nanomedicine applications but even in the broader robotic context. If we consider the basic components of a generic robotic system i.e sensory system, control system and actuation system, the MTB perfectly complies them. A simple schematic of the bacteria highlighting each component is presented in Figure 19.
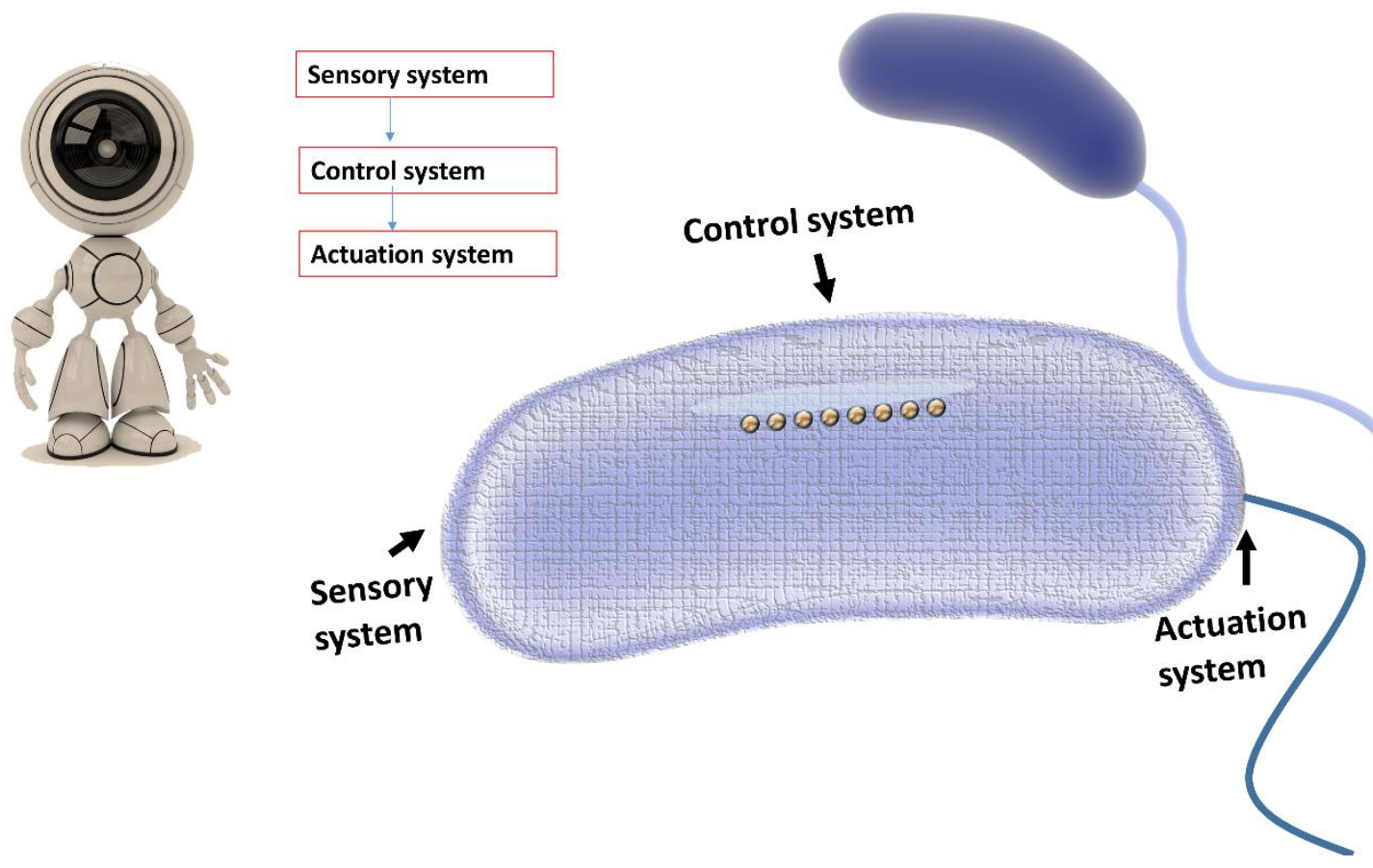

Figure 19. Schematic showing the basic components of a robotic system and highlighted in a magnetotactic bacteria. 


\section{WILEY-VCH}

Overall, to use the bacteria as a microrobot or bacteriabot, there is thus a need to understand their sensing mechanism and control their motility (Taxis) remotely. In addition, their detection is needed for analysis purposes. An overview of engineered systems and strategies for this end are given in the following section. In addition, as for closed loop control, the magnetic moment is of importance. Accordingly, a rapid overview on different ways to measure the magnetic moment is provided. For application purposes, loading a cargo is essential and therefore works with different functionalization strategies are also discussed.

\subsection{Microrobotic applications of MTB}

For utilizing the MTB in technological application, a key feature needed is the ability of steering them from a remote distance. Many of the pioneering work on developing a programmable guiding system for MTB for applications in robotics/drug delivery is done by a well-designed magnetic guiding system. For example, it was shown that using a custom-made electromagnetic grid it is possible to manipulate the MTB in a programmable fashion, to make them follow paths to manipulate other objects. ${ }^{[145]}$ MTB can be programmed to pick-up a microbead and to move along an externally programmed magnetic field line. For this, a mixture of MTB and microbeads is dispersed over a glass slide and by applying thereafter a current makes $1 \%$ of the bacteria to attach to the microbeads and start pushing after 5 minutes.

In vivo experiments in the carotid artery of a living swine, show that the MTB can propel and steer a ferromagnetic bead of $1.5 \mathrm{~mm}$ in vivo using a magnetic resonance imaging platform (MRI). ${ }^{[146]}$ Controlled MRI propulsion and steering of MTB strain MC-1 has been shown, this method allows additionally to visualize the MTB. ${ }^{[130]}$ This research was the first report on how to control MTB without addition of chemicals. Many works have since then been published which used similar MRI guiding systems. The most important is perhaps the one where MTB deliver drug-containing nanoliposomes to localized tumor hypoxic regions by the MRI guiding system. ${ }^{[84]}$ 


\section{WILEY-VCH}

Other approaches to generate magnetic fields as guiding systems has been studied by combining Helmholtz coils and Maxwell coils, using permanent magnets or by customized electromagnetic coils. For all of these, a closed-loop control system is required for accurate positioning and different models predict the relation between the applied current and generated field and can be categorized by their actuation. ${ }^{[147]}$ However, common methods like the linear proportionalintegral-derivative (PID) method does not take in to account uncertainties and nonlinearities of the microrobot motion. A robust control of a given microrobot trajectory with no chattering to follow step inputs was done by the implementation of a time-delay-estimation model as method to enhance the closed-loop control system.${ }^{[148]}$ Despite this progress, when testing in mice by injecting a swarm of bacteria, only a low percentage of bacteria was found around the targeted tumor. Many cells stray off due the heavy current and never reach calmer capillaries. A further improvement on closed loop control systems for point-to-point positioning of microrobots is to introduce a null-space control system to closed loop system. ${ }^{[149]}$ The MTB is guided to a reference point and its magnetic moment is obtained by the U-turn approach under reversal of the magnetic field and used to readjust its position. Thus, first the MTB orientation is controlled and thereafter a field with alternating directions is applied to decrease the MTB velocity allowing thus to control the MTB positioning. This approach allowed to control the MTB along a microfluidic maze. ${ }^{[150]}$ Antibiotics were successfully delivered to a an infectious biofilm by a MTB powered bio hybrid composed of MSR-1 integrated in a silica microtube. ${ }^{[135]}$ This biocompatible encapsulation allowed the successful cargo loading of antibiotics and the MTB motility increased the effective targeting disrupting the biofilm. 


\section{WILEY-VCH}

\subsubsection{Other Robotic Applications}

\section{Actuators}

In digital microfluidics (DMF), discrete droplets containing samples and reagents can be controlled to implement a series of operations via electrowetting on dielectric, magnetic, surface acoustic wave, and other stimuli, depending on the mechanism of droplet actuation. ${ }^{[151]}$ This is typically works on (super)hydrophobic surfaces. On the contrary to microchannel, DMF provides simple and precise control over multiple reaction processes in where each droplet is actuated independently allowing multiple functionality. Their applications span from chemical and enzymatic assays, immunoassay, cell based-operation (culture, sorting and purification), DNAbased applications (extraction, purification, amplification and detection) and protein analysis. ${ }^{[152]}$ MTB are used to provide actuation of aqueous droplets on a superhydrophobic surface by using for DMF. ${ }^{[153]}$ A whole drop containing MTB can follow a set of trajectories and effectively apply DMF to perform a microfluidic phosphatase assay. Here the MTB in addition acts as a source of enzymes that generates a color reaction when the bacteria was merged and mixed with a droplet containing the substrate (p-nitrophenyl phosphate) to analyze.

\section{Self-Assembly}

The MTB self-assembly process can also be a tool for robotic applications. ${ }^{[154]}$ For this, understanding the role of the hydrodynamics and magnetism involved is essential. The hydrodynamically self-assembled magnetic bacteria orients perpendicular to a surface and are selflimiting, reaching a quasi-static state. This phenomenon leads to the analogue observed in inactive colloidal systems called "self-focusing regime". Therefore, it can be used in a more general model for the design of self-assembled systems, allowing stable structure formation in designed artificial systems. In addition, MTB clusters may be directed along controlled paths or distorted under the 


\section{WILEY-VCH}

influence of in-plane fields. This allows to controllably assemble / disassemble MTB clusters and potentially use them to transport cargo to specific locations.

More information on the progress of different guiding system for drug delivery, magnetic manipulation and actuation are available in the following chapters.

\section{Bio Hybrids}

Many researchers are inspired by microorganisms and try to mimic their main properties or combine microorganisms with other engineered strategies to form so-called bio hybrids. Specifically the swimming capabilities of bacteria at low Reynolds number, in where viscous forces dominate, are of great interest. Combining a biological component with another material to modify their properties is in fact present in some of the works previously presented. For example the bio hybrid system of the MTB in a silica microtube that allowed facile cargo loading, antibiotics, and its targeted transport to disrupt a biofilm. ${ }^{[135]}$ Another MTB based bio hybrid is the system in where AMB-1 was combined with iron oxide nanoparticles to change its magnetic properties and ease the control of the MTB magnetotaxis. ${ }^{[155]}$ The functionalization herein was of great interest, as we will discuss later on. Besides these examples, there are many more bio hybrid systems with magnetic control, which have successfully been achieved and some are highlighted in the following.

We shortly discussed the role of the bacteria flagella earlier in this section, where a molecular motor rotates the helical flagella for forward propulsion. In microalgae (as in all eukaryotes), the two flagella produce planar waves, such as breaststroke waves in $C$. reinhardtii, by bending. ${ }^{[156]}$ An increase up to $100 \%$ efficiency is proposed for the planar wave compared to the single helical flagella. ${ }^{[157]}$ However, the microalgae lacks magnetic properties and therefore an adequate steering mechanism for its usage in robotics is missing. An approach to provide magnetic properties to the microalga species $C$. reinhardtii is to culture them in a media containing terbium ions $\left(\mathrm{Tb}_{3}{ }^{+}\right) \cdot{ }^{[158]}$ These ions are known to possess magnetic properties and to present photoluminescence and could 


\section{WILEY-VCH}

therefore potentially be used as a biomarker. The grown microalgae presented superparamagnetic properties and their motion could be controlled by magnetic fields and tracked by their photoluminescence.

Another MTB inspired robotic micro-swimmer was designed by isolating flagelar filaments from Salmonella typhymurium and magnetic nanoparticles. ${ }^{[159]}$ By using avidin-biotin linkages, a magnetic nanoparticle was conjugated to a polyterene microbead. The construction consisted of 3 phases. First, biotinylated monomers were utilized in a flagella repolymerization reaction to produce flagellar filaments that had biotin groups at both ends of the filament. Secondly, the microbeads and magnetic nanoparticles were functionalized with avidin. In the last step, all components were mixed yielding the magnetic biomimicking micro-swimmer. Their motion could be controlled by a set of Helmholtz coils.

Magnetic bio hybrid capable of moving large superparamagnetic beads are designed by randomly attaching multiple Serratia marcescens (S. marcescens) bacteria a $6 \mu \mathrm{m}$-diameter streptavidin coated superparamagnetic bead through streptavidin-biotin binding. ${ }^{[160]}$ The modified beads where added to the bacteria motility medium, mixed with LB, and incubated for 5 miniutes to allow for bacterial attachment to the beads by means of surface protein binding to the biotinstreptavidin bead coating. Remote control of their motion was successfully shown by applying weak magnetic fields.

Motivated by developing new fertilization methods, where the transport of a single spermatozoon to the egg cell location is required, successful encapsulation of a motile sperm cell in a $\mathrm{Ti} / \mathrm{Fe}$ microtube allow to construct a magnetic controllable sperm cell (spermbot). ${ }^{[84]}$ The bio hybrid consists on sperm cells within rolled-up magnetic microtubes, which are fabricated by rolling up thin ferromagnetic layers. The spermbot can penetrate cells and its motion directed by applying external magnetic fields. Different design of spermbot allow them to be used also in drug delivery. ${ }^{[161]}$ For this, the used microtube is a tetrapod, made of a polymeric structure, designed 


\section{WILEY-VCH}

and fabricated by two-photon 3D nanolithography. The tetrapod microstructures are asymmetrically coated with iron for magnetic control and titanium to make it biocompatible. The sperm can load a cargo and its motion allows higher efficiency killing spheroids HeLa cells. Yet a different spermbot design, the gelatin spermbots allows their use for theranostics application in reproductive biology. Here magnetic nanoparticles are added to the gelatin based encapsulation allowing to control the motion (the gelatin is propelled by the sperm flagella), ${ }^{[162]}$ other spermbot designs are also used in several robotic applications. ${ }^{[163]}$

\subsection{Determining the Magnetic Moment of MTB}

Determining the magnetic moment of MTB is of interest for closed loop applications as the region of convergence (which is the region around a reference point of interest), depends directly on the magnetic dipole moment. ${ }^{[164,165]}$ The larger the dipole moment, the higher the positioning accuracy through the closed loop control will be. There exist several techniques for the MTB magnetic moment determination, and could be categorized as indirect and direct magnetic moment measurements. ${ }^{[103]}$

\subsubsection{Indirect Measurements}

The counting of magnetosomes, by direct visualization with electron microscopy, and knowing the magnetic moment per unit of volume of the magnetic material allows estimating the total magnetic moment:

$M_{\text {total }}=n_{M a g} \cdot V_{M a g} \cdot M_{V}$

Where, $n_{M a g}$ is the number of magnetosomes, $V_{\text {Mag }}$ corresponds to the volume of each magnetosome and $M_{V}$ corresponds to the magnetic moment per of volume of the magnetic material. For the case of Magnetite $M_{\mathrm{V}}=480 \cdot 10^{-3} \mathrm{Am}^{2} / \mathrm{cm}^{3}$. 


\section{WILEY-VCH}

Due to the sizes of the magnetosomes, they act as superparamagnetic particles or stable single domain (SSD) ferrimagnets. ${ }^{[166]}$ When an external magnetic field is applied to these magnetosomes, their average magnetization orientation follows the average in fluctuations along the cosine of the angle between bacteria velocity and external applied magnetic field. Mathematically, this is described by the Langevin function that relates the bacteria magnetic moment and applied field with the thermal fluctuations. In this way by analyzing either the velocity or orientation of a single bacterium, its magnetic moment is estimated.

Another indirect way is by analyzing the U-turn the bacteria performs when applying an external magnetic field and changing the direction (Bean model). In this case, exactly at the point where the bacteria trajectory is changed, the sum of magnetic torque and viscous torque are equal in magnitude when considering low Reynolds number and ignoring flagellar forces. The helical radius (L) is determined as a function of the magnetic field, particle shape and magnetic moment. In addition, the turning time $(\tau)$ as a function of the magnetic field can be calculated and compared to the experimental observed turning time to validate the magnetic moment or to determine the same.

$$
\tau=\frac{8 \pi \eta R^{3}}{m B_{0}} \ln \left(\frac{2 m B_{0}}{k_{B} T}\right)=\frac{L}{\pi v_{0}} \ln \left(\frac{2 m B_{0}}{k_{B} T}\right)
$$

Here, $B_{0}$ is the magnetic field, $R$ is the radius of the cell, $\eta$ is the viscosity $k_{B}$ is the Boltzmann constant, $T$ is the temperature and $m$ is the cell magnetic moment.

Using this method D. M S. Esquivel and H. G. P. Lins de Barros analyzed a set of MTB. ${ }^{[167]}$

Based on the same method, by applying rotating fields and finding the maximum frequency $\left(\mathrm{f}_{\mathrm{C}}\right)$ for which the bacteria maintains its circular trajectory it is possible to calculate the magnetic moment as they relate by the following equation:

$$
M_{\text {total }}=c \eta 2 \pi f_{C} l^{3} / H
$$




\section{WILEY-VCH}

In where, $H$ is the applied magnetic field intensity, $c$ is the shape factor, $\eta$ is the viscosity and $l$ is the bacterium length. This method allowed to provide the magnetic moments of MYC-1 MTB strain. $^{[168]}$

Of course, alternative methods and other parameters can be extracted by analyzing bacteria trajectories and are encouraged to be revised also. ${ }^{[169]}$

\subsubsection{Direct Measurement Techniques}

The superconducting quantum interference device (SQUID) allows direct determination of the magnetic field generated by a given element. It consists in two superconductor magnets separated by an insulating layer, when a DC current is applied to the device, the voltage oscillates with a frequency that is proportional to the change in magnetic flux passing the device. Counting the oscillations allows calculating the flux change, which has occurred. The SQUID is a reliable method for determining the average magnetic moment of small samples of magnetotactic microorganisms collected directly from the environment. ${ }^{[170]}$

Another technique that allows direct detection of the magnetic moment is the vibrating sample magnetometer (VSM). Here, as indicated by its name, the sample of interest is vibrated in a detection coil space. The induced voltage in the coil is proportional to the magnetic moment following Faraday's law of electromagnetic induction. The sensitivity is typically around the $10^{-6}$ emu. The VSM easy usage and setup allows to study samples in different configurations such as the MTB, AMB-1, dispersed in a silica gel matrix. ${ }^{[171]}$ The immersion in a silica gel allowed constraining the role of an external applied magnetic field on the alignment of magnetosome chains. This permits to study reorientation of the chains within living magnetotactic bacteria exposed to an external magnetic field directly. A stable deviation is observed of the chain orientation within living cells suggesting that the assembly of cytoskeletal proteins are the responsible for the regulation of the nanocrystal organization and possess a dynamic character. 


\section{WILEY-VCH}

Optical techniques are used in where the optical properties changes are proportional to the presence or changes of magnetization of the sample. In general, these optical measurements have fast, reliable output and are relatively less expensive compared to the SQUID or electron microscopy. Near field scanning optical microscopy (NSOM) together with the magneto-optical Kerr effect (MOKE) can be used to visualize and analyze the magnetic properties of the MSR-1 bacterial cells. ${ }^{[172]}$ The combined techniques allows visualizing and magnetically characterizing both magnetosomes and cells directly. Light scattering can be used to determine average lengths and magnetic moments of the MS-1 bacteria. ${ }^{[173]}$ Obtained MTB magnetic moments by this technique are comparable with those obtained from electron microscopy. Magnetic induced birefringence also allows to determine the magnetic moments of MTB. ${ }^{[174]}$ The techniques is a fast and reliable method and comparable to results obtained by light-scattering determinations and estimates made from electron microscopy. The orientation of the bacteria, upon applied magnetic field, follow an angular distribution, which affects the structure factor in the scattered light intensity. This phenomena was used to characterize south and north seeking MTB in both AMB-1 and MO-1. ${ }^{[88]}$

\subsection{Loading a Payload/Cargo to MTB}

Another critical step toward the efficient use of MTB for delivering therapeutics or nanorobotics is to develop a method of loading the bacteria while maintaining its overall properties unchanged. In this sense, the modification of MTB with nanoliposomes for MRI targeted drug delivery had great impact ${ }^{[84]}$ and the MTB encapsulated in the silica microtube ${ }^{[135]}$ also allowed to maintain the MTB properties. However, the modification of MTB with nanoliposomes had been accomplished before by covalent binding through carbodiimide chemistry. ${ }^{[175]}$ In this case, amine-containing molecules of bacteria covalent bind to carboxylated liposomes. Also, other cargo binding to bacteria based microrobot are done through immunoreactions. ${ }^{[176]}$ Whereas, the work discussed in the bio hybrid section in where $\mathrm{Fe}_{3} \mathrm{O}_{4}$ magnetic materials are deposited on the MTB surface was even achieved through electrostatic interaction and shown that the overall MTB surface charge is 


\section{WILEY-VCH}

negative allowing to effectively immobilizing positively charged molecules. ${ }^{[155]}$ MSR-1 could so be modified with positively charged DNA coated gold nanoparticles to mimic transmembrane proteins. Increasing the loading efficacy and as a means of visualization. ${ }^{[177]}$ Several attachment strategies via physical synergies are thus available and in general they can be classified by hydrophobic/electrostatic interaction, inherent response and chemical interaction such as streptavidin, biotin and covalent binding. ${ }^{[104,105]}$

\subsection{Scope}

Although many works have shown potential usage of MTB in robotics, there still are many open questions and space for improvements. For example, in the case of drug delivery applications, we could ask ourselves what concentration of bacteria would be needed to effectively eradicate the tumor? In addition, how cytotoxic are these bacteria actually? Also, when used in the human body, will a new immune defense rise? Recent study on magnetosomes with HELA cell have shown promising results regarding their cytotoxicity showing biocompatibility and suffering no chemical nor physical changes after internalization by the cells. ${ }^{[178]}$ Magnetosomes degradability in human stem cells has also recently been studied showing that after degradation from magnetite to ferrihydrite, the human stem cell are re-magnetized entirely biosynthesizing magnetic nanoparticles anew forming again magnetite. ${ }^{[179]}$

More generally, the release of a given cargo is still not well controlled although there exist many promising strategies their actual application is limited or not yet tested on MTB. ${ }^{[180,181]}$ Regarding the steering mechanism, upon a given application other approaches based on the sensory mechanism, like ratchets ${ }^{[182]}$ or locally, at the microenvironment, control oxygen gradients. ${ }^{[183]}$ Regarding their magnetic properties, interaction with other materials like antiferromagnetic nanomaterials could bring new behaviors into light. Theoretically it is shown that MTB might present original magneto-rheological properties and present novel collective behavior. ${ }^{[184-187]}$ Experimentally, the MTB were shown to behave as rotary motors in oil-water emulsions for 


\section{WILEY-VCH}

example and therefore their behavior in complex medium is an open field. ${ }^{[188]}$ In this line, taking into account that among MTB strains they have different properties and needs, the same diversity is most likely to be translated to different collective behaviors which if controllable are of interest for the field or robotics.

\section{Magnetic Biohybrid Cellular Micro-Bio-Robots [Magdanez]}

Downscaling macroscopic actuation mechanisms to create microscale motion is often not feasible due to the limitation in fabrication methods and the difference of physical phenomena governed by low Reynolds number hydrodynamics on the microscale. Biological systems have evolved over millions of years to move and function in an optimized way on the microscale. Thus, biohybrid systems have become attractive approaches during the last decade to develop magnetic microrobots from several different point of views. Evidently, for targeting biomedical applications, biocompatible microrobots are required which can propel, function and be controlled under physiological conditions. For this reason, biohybrids are promising options. Further, cells are known to respond to their environment by many sensing and taxis mechanisms. As described in a previous review, the biological components can serve as various functional units, namely as templates, loading units or propulsion units. ${ }^{[189]}$ Nanotechnology has allowed the integration of biological components as templates to create novel magnetically controlled biohybrid systems. Plant-derived structures have served as a basis to fabricate drug carriers that are magnetically actuated or guided. ${ }^{[190-194]}$ Further, flexible magnetic micro-swimmers were created on the basis of bovine sperm cells, covered with magnetic nanoparticles. ${ }^{[195,196]}$

Regarding the magnetic control, we can distinguish between directional control and actuating control. If the biohybrid microrobots display self-propulsion by other means (as in the case of motile cells such as sperm cells or bacteria), only weak, constant magnetic fields are needed to introduce magnetic directional guidance. In contrast, when the magnetic fields serve as driving 


\section{WILEY-VCH}

source, different configurations of magnetic fields have been employed. Here, planar oscillations or three-dimensional rotating magnetic fields with various cone angles are applied.

In this section, we will summarize the advances of magnetic driven microrobots based on the integration of spermatozoa or bacteria.

\subsection{Sperm-based biohybrid microrobots}

Spermatozoa were incorporated as propulsion sources or as templates for flexible magnetic swimmers, as illustrated in the following two sections. Spermatozoa are male gametes which swim to the egg cell with the help of their powerful flagellum. Their motility is based on the orchestrated action of molecular dynein motors, which bend and release the microtubule all along their axoneme. This leads to a bending wave motion of the tail, while the head is passively moved forward. Thanks to the strong propulsion force of sperm, they have been applied as power sources of hybrid microrobots. Lately, due to the intrinsic flexibility of the sperm cells, they have also been utilized as templates for magnetic microrobots.

\subsubsection{Sperm-driven microrobots}

Ferromagnetic microtubes offer a method for the capture and remote control of motile cells. By the use of strain engineering, $50 \mu \mathrm{m}$ long rolled up nanomembranes were tuned in size to fit single bovine sperm cells. ${ }^{[197]}$ The $60 \mu \mathrm{m}$ long sperm cells randomly entered the rolled up microtubes, became mechanically trapped and started pushing the microtubes forward. The rolled up nanomembranes contained a nanometer-thin iron layer, which enabled the magnetic remote directional control of the sperm-driven microrobots by small magnetic fields of just a few milliTesla. ${ }^{[198]}$ In comparison to free sperm, the sperm-driven microtubes displayed a velocity reduced by around $80 \%$ due to the physical confinement of the cell, which restricts the flagella bending motion. In order to improve the performance of such hybrid microrobots, shorter rolled up microtubes $\left(20 \mu \mathrm{m}\right.$ long) were fabricated and used for the coupling with the sperm cells. ${ }^{[199]}$ 


\section{WILEY-VCH}

This maintained a higher velocity of the biohybrid robots, but the coupling success was lower due to sperm cells being able to escape through the short microtubes more frequently. Next, surface functionalization methods were applied to bring sperm-binding proteins onto the inner surface of the microtubes for increased sperm binding. ${ }^{[199]}$ This achieved a higher coupling success rate between the sperm cells and microtubes. Sperm cell release was implemented by incorporating a thermoresponsive polymer into the $\mathrm{Ti} / \mathrm{Fe}$ microtubes. This enabled the opening and thereby releasing of the cells by a small temperature increase while also offering magnetic directional control. ${ }^{[200]}$ In another approach, 3D printed polymeric microstructures were coated with an iron and titanium layer and used for capturing drug-loaded bovine sperm. ${ }^{[201]}$ While the sperm cell was the propulsion source, weak magnetic fields were used for directional guidance to cancer spheroids demonstrating the suitability of the system for drug delivery applications. The 3D printed microstructure contained a four-armed front structure which offered a mechanical cell release mechanism. Recently, gelatin-based microcartidges were employed for sperm manipulation. ${ }^{\text {[202] }}$ The magnetic nanoparticle-containing microstructures were fabricated by template-based polymerization of gelatin inside the pores of a polycarbonate membrane. The resulting microcartidges captured single sperm cells, which propel the structures under directional guidance of weak magnetic fields. These microcartridges were loaded with heparin, a sperm-activating agent that induced capacitation, a crucial sperm maturation step prior to fertilization. These gelatin structures also showed a controlled biomolecule release upon $\mathrm{pH}$ change and a reactive oxygen scavenging effect. The latter is an important protection against reactive oxygen species, one of the main causes of sperm damage during in vitro handling.

\subsubsection{Sperm-carrying microrobots}

Non-motile sperm cells can be motorized by capturing them in tailored microhelices and applying rotating magnetic fields for their forward propulsion. ${ }^{[203]}$ The microhelices were fabricated from photoresist by 3D nanolithography and subsequently coated with nickel. This enables a precise 


\section{WILEY-VCH}

rotational magnetic actuation as well as forward and backward screw-like motion allowing the pick-up and release of the non-motile sperm cells. This approach is especially promising for the delivery of non-motile, but viable sperm to the oocyte. This method has prospects in assisted reproduction to treat certain types of asthenospermia (low or no sperm motility).

\subsubsection{Sperm-templated microrobots}

The flagellum of spermatozoa has an intrinsic flexibility with a bending stiffness that varies along the length of the tail. This has inspired researchers to use immotile sperm cells as templates for the facile fabrication of flexible magnetic micro-swimmers. The fabrication is based on the electrostatically driven self-assembly of magnetic micro-or nanoparticles and the sperm's surface. This resulted in different amount and localization of magnetic particles on the sperm's membrane. First, planar, undulating magnetic fields were applied with the use of four electromagnetic coils. $^{[195]}$ This resulted only in small forward velocity. In addition, the flexibility of the sperm template could not be maintained in an optimal way, because $1 \mu \mathrm{m}$ microparticles impaired the flexibility of the sperm tail. In a next study, smaller, elongated $100 \mathrm{~nm}$ iron oxide particles were used for the self-assembly with the sperm. This maintained the flexibility of the sperm tail in an improved fashion. ${ }^{[204]}$ The application of a three-dimensional rotating magnetic field resulted in flexible bending motion of the magnetic sperm-templated swimmer. The applied frequency and cone angles can be used to control their forward motion and overall performance. When comparing the swimming mechanisms of the magnetically actuated sperm versus the motile sperm, the resulting waveforms differ from each other. ${ }^{[196]}$ The magnetically actuated sperm are characterized by lower linearity, higher asymmetry and lower forward progression than their motile counterparts. The thrust force calculated by resistive force theory is higher in the case of the motile cells compared to the magnetically actuated sperm. This leads us to the conclusion that there is still much to learn from the biological motion mechanisms of spermatozoa. The study of such systems is useful for improving the design of flexible biohybrid microrobots. 


\section{WILEY-VCH}

\subsection{Bacteria-Driven Biohybrid Microrobots}

Similar to sperm cells, motile bacteria have been integrated as propulsive forces of hybrid microrobots. Bacterial motility types is diverse, ${ }^{[205-207]}$ but for biohybrid propulsion, mostly flagellated bacteria have been employed. Prokaryotic flagella have a rotor on its base, anchored in the membrane of the bacteria. The propulsive force is generated by a molecular motor complex converting a proton gradient into the rotational motion of the bacterial flagellum that spins around a central axis. Bacteria display various taxis abilities which inspired microrobotics researchers to employ them as guidance and control mechanisms. Taxis mechanisms of bacteria include response to gradients of substrates, oxygen, $\mathrm{pH}$, temperature, light or magnetic and electric fields. For the control of microrobots, thus far, chemotaxis, ${ }^{[208-211]}$ aerotaxis, ${ }^{[212]}$ magnetotaxis ${ }^{[212-214]}$ and $\mathrm{pH}-$ taxis $^{[215]}$ have been explored. A unique role play magnetotactic bacteria which display reorientation within magnetic fields due to their intrinsic magnetic moment created by their magnetosomes (see section 3: magnetotactic bacteria). Another nice feature of using bacteria as components of microrobots is the ability to genetically modify them easily to obtain certain desired properties.

In most bacteria-driven microsystems, the motile cells were attached to the artificial components by functionalizing the particle's surface to achieve receptor-based covalent coupling to the cell. E.coli was applied as driving source of bacteriabots by attaching them to polyelectrolyte multilayer microparticles. ${ }^{[216]}$ Another attachment route is charge-based interaction, e.g. binding positively charged microobjects to gram-negative bacteria. ${ }^{[217]}$

The guidance mechanisms of bacteria-driven microbots are vast, but here we restrict the scope to cases that apply magnetic guidance. Analog to the sperm-driven microtubes, bacteria were captured inside magnetic microtubes. ${ }^{[218]}$ Here, the magnetic microtubes were fabricated by electrodeposition onto a polycarbonate membrane, resulting in polypyrolle microtubes containing 


\section{WILEY-VCH}

nickel nanoparticles and polydopamine. In a planar setup of electromagnetic coils, E.coli bacteria were transporting the microtubes and guided directionally by weak magnetic fields. Further, Janustype bacteriabots were fabricated by attaching E.coli to the iron caps of polystyrene particles. While the bacteria propelled the drug-loaded particles, weak magnetic fields could be used to reorient the bacteriabots. ${ }^{[219]}$ Multiple bacteria were attached to superparamagnetic microbeads to decrease the stochasticity of motion and thereby enhance the magnetic steering controllability. ${ }^{[220]}$ Other types of microorganisms such as algae have also been employed as propulsion sources of magnetic microrobots. Chlamydomonas reinhardtii propels attached microobjects with two flagella. ${ }^{[221]}$ Microalgae such as Spirulina platensis have been utilized as templates for magnetic helical swimmers. ${ }^{[192,222]}$

All here described types of cellular biohybrid microrobots display promise for translation into clinical scenarios, such as minimally invasive therapies for cancer treatment, biopsy and cell manipulation. ${ }^{[207]}$

\section{Electromagnetic Soft Actuators [Ebrahimi]}

Electromagnetic actuators (e.g. DC motors, solenoids, voice coil motors, etc) are, by far, the most widely being used type of actuators in the traditional robotic applications. This is due to their ease of operations, simplicity in control and efficiency in converting electrical power into mechanical actions, but they are made of rigid elements. The problem is, rigidity in the materials of an actuator limits its performance in many robotic applications, especially when the robotic platform is supposed to physically interact with humans. In these scenarios, in order to guarantee safety of humans with whom the robot is physically interacting, the body of the robot, an especially its actuators as sources of providing energy, should be soft. The question is how to make an electromagnetic actuator with soft materials. 


\section{WILEY-VCH}

\subsection{Fabrications}

In order to fabricate electromagnetic soft actuators, the working principle and essential components of such actuators have to be identified and then realized with soft matters. The fundamental working principle of all electromagnetic actuators is based on interaction forces between two magnetic fields: a magnetic field due to presence of a permanent magnet and another magnetic field created by an electromagnet (i.e. current through a conductive coil).

Therefore, in order to build a soft electromagnetic actuator, we need to realize a permanent magnet as well as a conductive coil with soft matters. In addition, we would need a soft material that can get magnetized when it is exposed to an external magnetic field and keep its magnetization for a while when the external magnetic field is removed, a property that is defined as permeability. In traditional rigid electromagnetic actuators, usually Iron is being used as a return path for the magnetic field as it has high permeability. A representative example of an electromagnetic actuator (Solenoid) ${ }^{[223]}$ is shown in Figure 20 with its fundamental components.

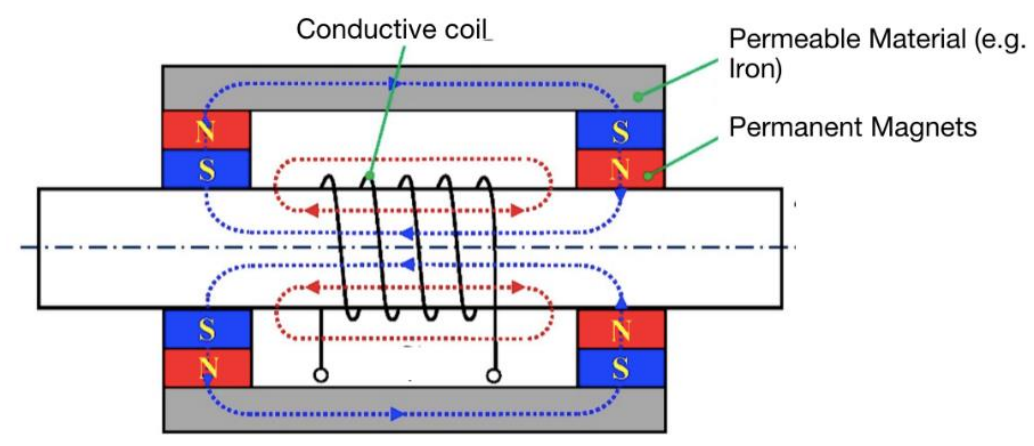

Figure 20. Fundamental components of a Solenoid (permanent magnet, permeable material and conductive coil ${ }^{[223]}$.

To realize conductive wire, researchers have used conductive liquids such as Eutectic Alloys Galinstan $(68 \% \mathrm{Ga}, 21.5 \% \mathrm{In} \text {, and } 10 \% \mathrm{Ti})^{[224]}$ and Eutectic Gallium Indium Alloy (EGaIn, 75\% Ga, 25\% In by mass) ${ }^{[225,226]}$. These liquids can be injected into soft micro-pipes or micro-channels made of rubbery-like materials, that are usually made of Polydimethylsiloxane (PDMS), also known as dimethylpolysiloxane or dimethicone ${ }^{[227,228]}$, belong to a group of polymeric 


\section{WILEY-VCH}

organosilicon compounds that are commonly referred to as silicones. The PDMS body of the micro-pipe or micro-channel plays role of an insulator for these soft wires. Micro-pipes can be realized using extrusion fabrication techniques or by a method Nho Do et al. ${ }^{[229]}$ where a thin layer of Ecoflex ${ }^{[230-232]}$ (a blend of recycled polymers and wood byproducts that exhibits characteristics of the polymers and wood) powder is laminated on a flat surface using a stainless-steel roller and then a fine carbon fiber rod is rolled onto the thin polymer layer. The laminated layer and rod are then heated and then the rod is pulled out and EGaIn is injected and then the whole filament will be formed as a coil (Figure 21).

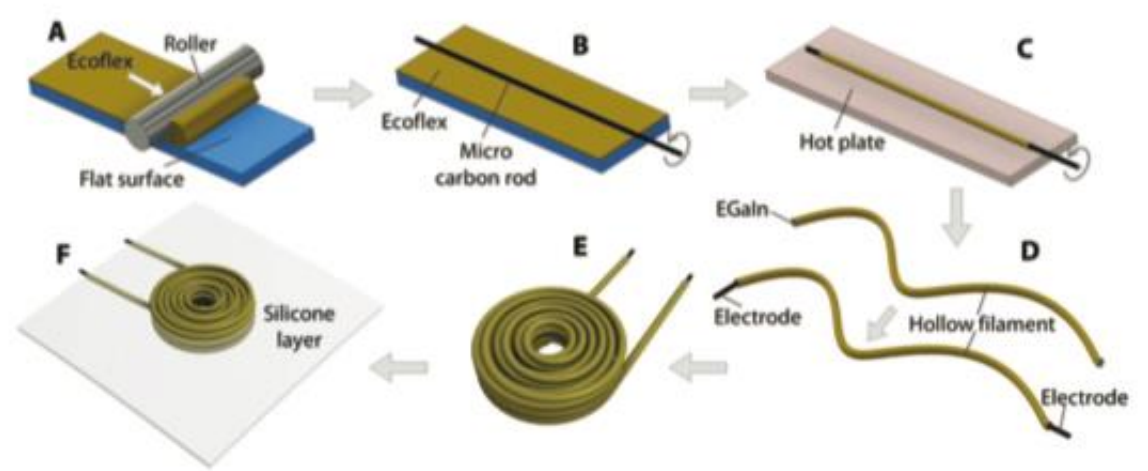

Figure 21. Fabrication process for the soft, 3D helical coil inductor. A) A thin layer of liquid silicone elastomer (Ecoflex 0030) is laminated onto a flat surface using a stainless-steel roller. B) A fine, carbon fiber rod is rolled onto the thin silicone layer ${ }^{[229]}$.

The other method to realize a soft conductive coil is creating micro-channels with $3 \mathrm{D}$ printing techniques ${ }^{[233,234]}$. The advantage of 3D printing micro-channels over the previously described method of realizing micro-tubes is achieving higher-dense, more compact coils with smaller crosssection area. This would lead to generating larger amount of forces as the electromagnetic force that can be generated is a function of number of the conductive loops. However, the fabrication of these micro-channels requires high-end 3D printers such as Nanoscribe ${ }^{[235,236]}$ that are extremely expensive. 


\section{WILEY-VCH}

Compared to traditional copper wire that is being widely used in almost any electromagnetic actuator, the use of conductive soft wire with conductive liquid inside PDMS has some limitations as well as some advantages. First of all, the specific resistivity of copper $\left(1.68 \times 10^{-8} \Omega \mathrm{m}\right.$, at $\left.20^{\circ} \mathrm{C}^{[237]}\right)$ is less than that of any conductive liquid, for example EGaIn $\left(24.9 \times 10^{-6} \Omega \mathrm{m}\right.$, at $\left.20^{\circ} \mathrm{C}^{[229]}\right)$. This means smaller input voltage is required to achieve same amount of electric current through a copper wire compared to a case for EGaIn-based soft wire at the same length and diameter, and therefore using copper wire, is more energy efficient way of generating electromagnetic force, as the force is function of electric current. However, copper wires are very sensitive to the magnitude of the current going through them. As the current increases, the heat increases and that can burn out very tiny insulator cover of the copper wires. However, using soft wire has more robustness against the generated heat, as EGaIn is already liquid with very high vaporing temperature ${ }^{[225]}$ and also PDMS has also very high melting point ${ }^{[238]}$. This means that the current limitation using a soft wire is much higher than that of a copper wire and as a result larger amount of force can be generated using soft wires, however, that would be at the cost of energy efficiency.

Fabrication of permanent magnets from soft materials is very challenging. Permanent magnets are usually made of from "hard" ferromagnetic materials such as Alnico and Ferrite ${ }^{[239]}$ that are subjected to special processing in a strong magnetic field during manufacture to align their internal microcrystalline structure, making them very hard to demagnetize ${ }^{[240]}$. Here, the word "hard" refers to ferromagnetic materials that can be easily magnetized but hardly get demagnetized, in other words they can keep their magnetism for a long period of time. The problem is magnetically hard materials such as Iron and Alnico, are also mechanically hard ${ }^{[241]}$. In order to make mechanically soft permanent magnets, there are basically two main techniques: extrusion and molding. The extrusion technique is usually used for fabrication of rubber magnets where the granular material (such as NdFeB granule neodymium) is heated until it starts to melt and then forced under high 


\section{WILEY-VCH}

pressure using a screw feeder through a hardened die where it is mixed with rubber. The die has been electrical discharged and eroded to have the desired shape. As materials continually flows from the die, it cools and passes over a magnetism fixture that magnetize the granule ${ }^{[242]}$.

Another method of fabricating soft permanent magnet has been proposed by Nho Do et al. ${ }^{[229]}$ (Figure 22) where a permanent magnet is hammered and crushed to powder. The powder is then mixed with liquid Silicone polymer and then poured into a 3D printed mold. The mold is then placed inside a strong external magnetic field so that the magnetic particles align during the curing process. Once completely cured, the magnetic particles will stay aligned even if the external magnetic field is removed. The advantages of this molding fabrication technique over the extrusion one, are :1- the direction of magnetic field with respect to the base material can be easily adjusted by simply tuning the direction of external magnetic field and 2- it is cheap and doable in a lab environment. However, the flexible magnets made through extrusion technique have stronger magnetic field. Nevertheless, the magnetic fields of flexible magnets ae not as strong as those of commercially available, traditionally hard magnets.

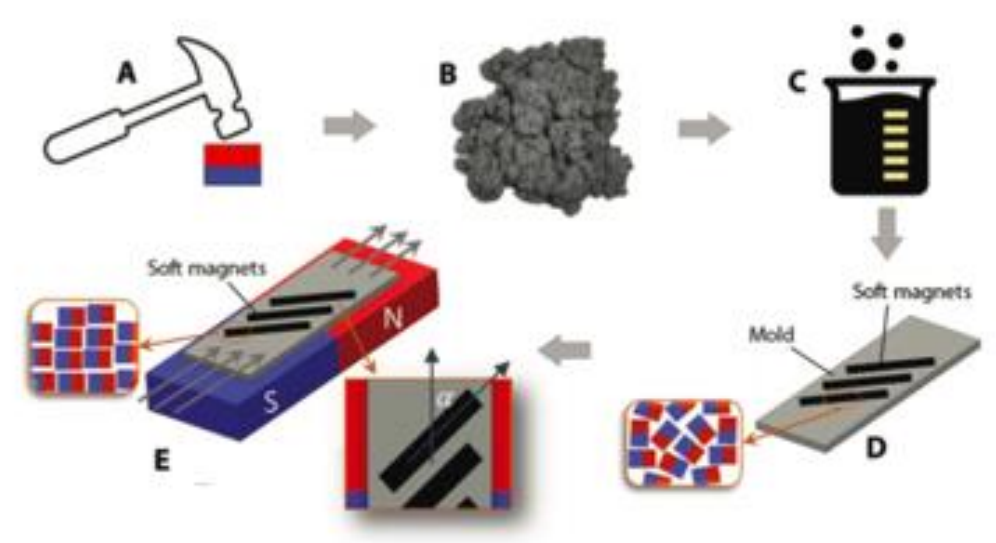

Figure 22. Fabrication process of soft permanent magnets: (A) crush a permanent magnet into (B) powders which then $(\mathrm{C})$ mixed with liquid silicone polymer using a mixture machine. (D) pour the suspension of magnetic particles and PDMS into a 3D printed mold and (E) align the mold above a permanent magnet to elicit a specified magnetization orientation ${ }^{[229]}$.

Fabrication of magnetically permeable soft materials can be simply done by replacing Iron oxide $\mathrm{Fe}_{3} \mathrm{O}_{4}$ particles with magnetic particles in the aforementioned molding process ${ }^{[243]}$. The existence 


\section{WILEY-VCH}

of the Iron oxide particles will allow the magnetic field to have a return path while being exposed to an external magnetic field. It is important to mention that; the permeability of these materials is obviously less effective than those used in traditional rigid electromagnetic actuators as the percentage of Iron is less in soft permeable materials. By increasing the percentage of Iron, the permeability will increase, however, the flexibility of the material will decrease. Ebrahimi et al. ${ }^{[24]}$ have experimentally tested different percentage of Iron oxide mixture with PDMS regarding tension force, maximum elastic elongation and yield point. It was concluded that around $35 \%$ of Iron oxide mixed with PDMS would lead to $70 \%$ increase in tension force to achieve same amount of elastic elongation, while the yield point is almost $80 \%$ as for a sample made of pure PDMS. Interestingly, adding only 5\% Iron oxide (i.e. $40 \%$ Iron oxide-60\% PDMS) to the mixture resulted in dramatically decrease in the yield point (less than $30 \%$ of pure PDMS sample), while tension force for the same amount of elongation as compared to a pure PDMS sample stayed at around $65 \%$. This means that adding more Iron oxide does not considerably affect the elasticity of the mixture but would make it very fragile.

\subsection{Applications}

Leon, et al. presented an application based on the bio-inspired motion of the Amoeba ${ }^{[245]}$. Their research was focused on development a soft micro robot based on the Amoeba locomotion which they called it: Whole Skin Locomotion (WSL) as it shown in Figure 23.
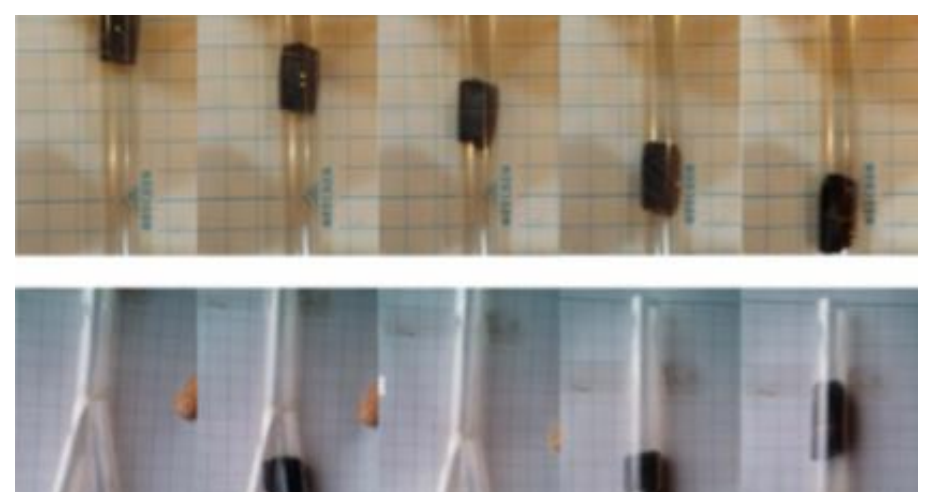

Figure 23. top: First trials along X axis of WSL robot; bottom: Steering motion of WSL robot ${ }^{[245]}$. 


\section{WILEY-VCH}

The WSL robot was created using the fluid filled toroid method that acts as a body shaping feature with Ferrofluid material ${ }^{[246]}$ placed within that is delivered to create the driving force. The passive fluid switch acts as an active sensitive liquid when a magnetic field is applied. Therefore, based on this behavior, in order to produce the driving motion, external electromagnetic coils were arranged as a wireless control and actuator. A number of motions and hindrances were then presented to insure the principal motions of the robot. Some other approaches of Ferrofluid softrobot biomimetic inspired were also presented as well.

Using the movement of one single cell like amoeba animals move implemented as a biomimetic soft robotic and using a Ferrofluid as passive/active actuator, the movement motion of WSL was confirmed through various experiments. Fluid-filled toroid in the human digestion system is a potential application for drug delivery system or if possible further miniaturization movement in the vessel is also expected to be achieved through the control of an external magnetic field in intravascular applications. As a main advantage of the WSL robot proved that is possible to achieve the two-dimensional movement and not a simple linear motion. Where, Ferrofluids can offer remarkable actuation response in soft robots for future applications and improvements. However, to obtain a precise control, it requires further analysis as nano particles for molecular motion. Also, the Ferrotluids could be considered as neutral monopole or magnetized dense liquid that follow the magnetic field with capillarity restrictions forces ${ }^{[247]}$.

Additional challenges arose from the tests, such as the difficulty to trace concentration material of Ferro-particles in specific areas in order to improve the performance and also create a micro buoyancy space to execute a tlotation motions its horizontal displacements for the underwater applications. However, micro channels as alternative of powered the Ferro-fluid is also possible in other to used its hydrodynamics properties.

Nho Do et al. developed and fabricated Soft Electro-Magnetic Actuators (SEMA) ${ }^{[229]}$, which are actuated based on the Lorentz force principle, via electrical current. The central innovation of their 


\section{WILEY-VCH}

devices was a soft electromagnetic inductor made from 3D helical coils formed from fine, stretchable hollow filaments that are filled with a liquid metal conductor. By fabricating these filaments from a colloid of silicone polymer and EGaIn microdroplets, they achieved high thermal conductivity, facilitating greatly increased current handling, and commensurately higher magnetic fields, and forces. Using these technologies, they demonstrated small scale cylindrical actuators capable of linear high-frequency motion as shown in Figure 24.

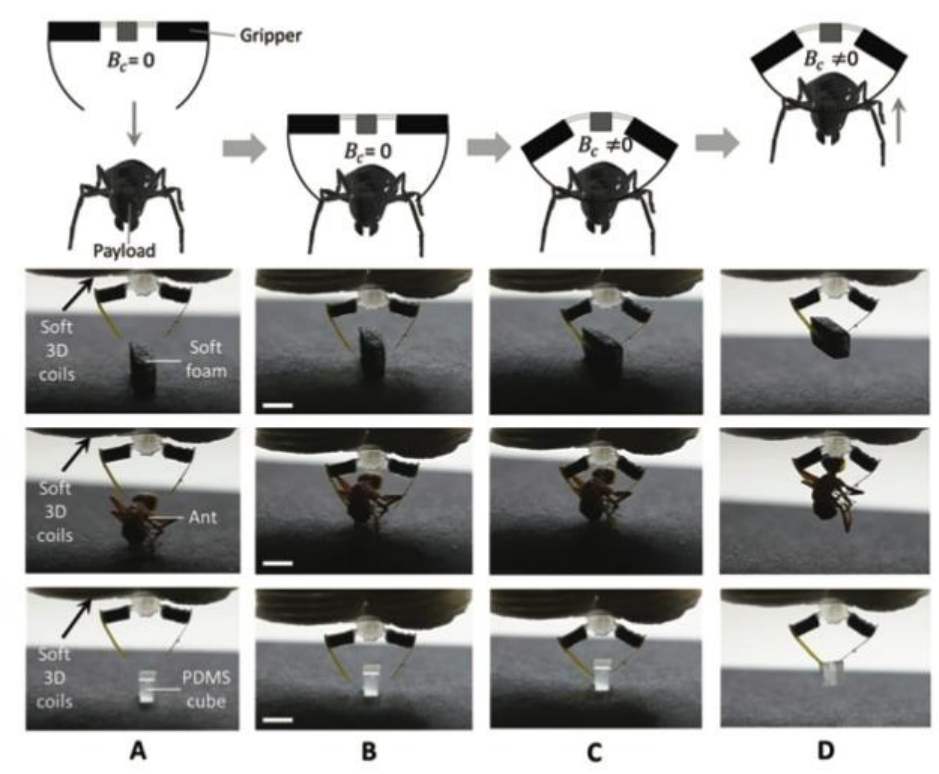

Figure 24. Manipulation process steps and experimental validation for the miniature gripper with a soft foam cube, an ant, and a PDMS cube. A) Initial position of the gripper. B) The gripper approaches vertically from the top to the payload. C) The gripper holds and lifts the payload. D) The gripper moves back to its original position. The objects are $\approx 2$ $\mathrm{mm}$ wide. Videos for real-time experiments are presented in the Supporting Information. Scale bar: $2 \mathrm{~mm}^{[229]}$.

The performance of these devices exhibited close quantitative agreement with predictions of a mathematical model based on the Lorentz force principle. They were capable of operating over a scalable range of voltages or currents, here ranging from $50 \mathrm{~mA}$ to $>1 \mathrm{~A}$, or $50 \mathrm{mV}$ to $1 \mathrm{~V}$, yielding displacements of up to $1 \mathrm{~mm}$, and retained most of their performance when stretched up to $100 \%$, or bent to angles of $38^{\circ}$. They applied these actuators in multipoint arrays, which are suited to 


\section{WILEY-VCH}

providing tactile feedback in wearable devices, even as they stretch to conform to the skin. They also demonstrated multi degree-of-freedom devices that are capable of articulated motion and demonstrate their application in a unique miniature soft robotic gripper, which proved capable of manipulating (grasping, lifting, and releasing) miniature loads.

The proposed SEMAs by Nho Do et al. have several key advantages: they were fast, capable of operating at high frequencies, they were operated at low voltages, were thermally efficient, enabling them to achieve high transient or sustained displacements. They were operated even when bent or stretched. They also offered theoretically predictable performance that was scalable in size and force, and finally, they were polymodal, suited to integration in simple arrays or articulated structures. These actuators shared a disadvantage that is common to other electromagnetic actuators, which is that higher forces require proportionally higher currents, with the feasible current limited by heating. As introduced by Nho Do et al., these devices mitigate this through the use of thermally conductive polymers that greatly reduced heating. They also have the disadvantage of relatively small displacements, although this could be improved through mechanical design. In a broader context, owing to the attractive properties (including stretchability, speed, ease of driving, and scalability), these actuators could prove useful in applications benefitting from integration in wearable electronics ${ }^{[248]}$, microsurgical robotic instruments ${ }^{[249]}$, soft $\operatorname{MEMS}^{[250]}$, acoustic actuators ${ }^{[251]}$, microfluidics ${ }^{[252]}$, and autonomous soft robotics ${ }^{[253]}$. They are more complex to fabricate than simpler pneumatic soft actuators, but are faster, simpler to drive with microelectronics, and easily adaptable to proportional control over a wide dynamic range. Rui et al. ${ }^{[254]}$ fabricated liquid metal electromagnetic actuators via liquid metal spraying technology to develop soft jellyfish robots as shown in Figure 25. 


\section{WILEY-VCH}

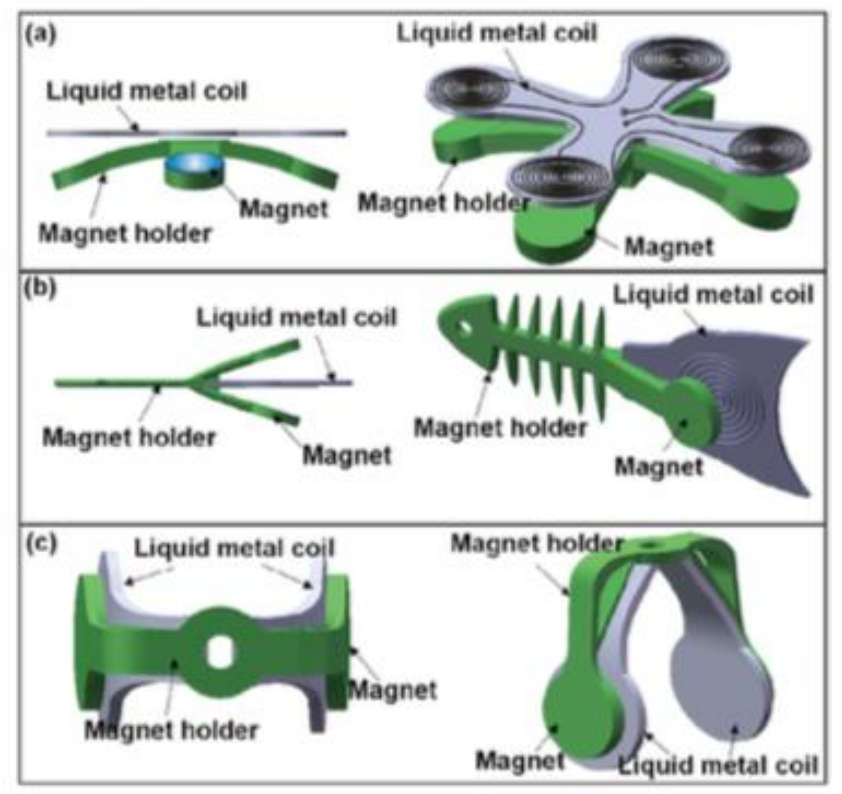

Figure 25. The structure of the soft mechanical devices. (a) the structure diagram of the jellyfish soft robot, (b) the structure diagram of the soft fishtail, (c) the structure diagram of the soft manipulator ${ }^{[254]}$.

In this method, they first spin a layer of PDMS membrane with thickness around $0.5 \mathrm{~mm}$ on a smooth Silicone wafer. The PDMS substrate was then cured in an oven. Subsequently, cover the film with a particular shape mask plate, using liquid metal spraying gun to print liquid metal evenly on the PDMS membrane. They then removed the mask plate, and made some holes with diameter of around $1 \mathrm{~mm}$ on the location of the coil endpoints and then filled the liquid metal in these holes. Finally, evenly spin coated a layer of PDMS membrane on the pattern and then cured the PDMS in an oven (Figure 26).

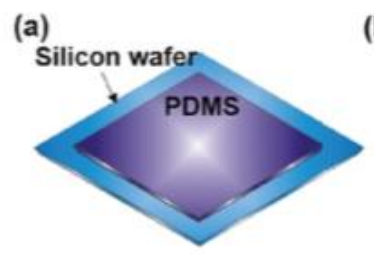

(d)

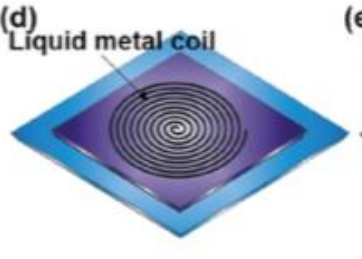

(b)

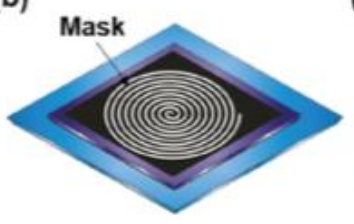

(e)

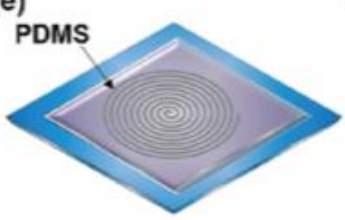

(c)

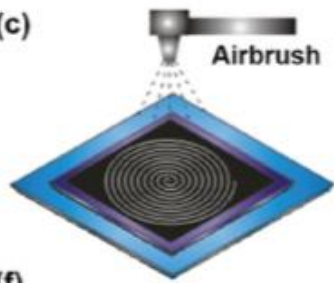

(f)

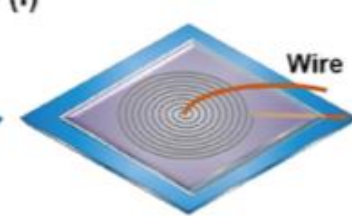




\section{WILEY-VCH}

Figure 26. Fabrication process of the liquid metal electromagnetic actuator based on the spraying technology ${ }^{[254]}$.

The preparation technology proposed by Rui et $\mathrm{al}^{[254]}$. showed evident advantages such as short production cycle and low cost. However, certain uneven distribution of liquid metal drops on PDMS membrane was noticed that required repeated spraying.

In their designs, the electromagnetic interaction between the magnet and liquid metal coil is the main driving principle of the actuators. However, due to the imitation of the electric current, the size of the coils and magnetic field intensity, the Lorentz force between the liquid metal coils and the magnets was very small. Nevertheless, this range of force was enough to propel the light weight of the robot.

McKenzie at $\mathrm{al}^{[255]}$. presented a soft, modular robots that were explicitly designed for manufacturability as they called them Linbots. Linbots use multifunctional voice coils to actuate linearly, to produce audio output, and to sense touch. When used in collectives, the Linbots can communicate with neighboring Linbots allowing for isolated behavior as well as the propagation of information throughout a collective. They demonstrated that these collectives of Linbots can perform complex tasks in a scalable distributed manner, and showed transport of objects by collective peristalsis and sorting of objects by a two-dimensional array of Linbots (Figure 27). 


\section{WILEY-VCH}

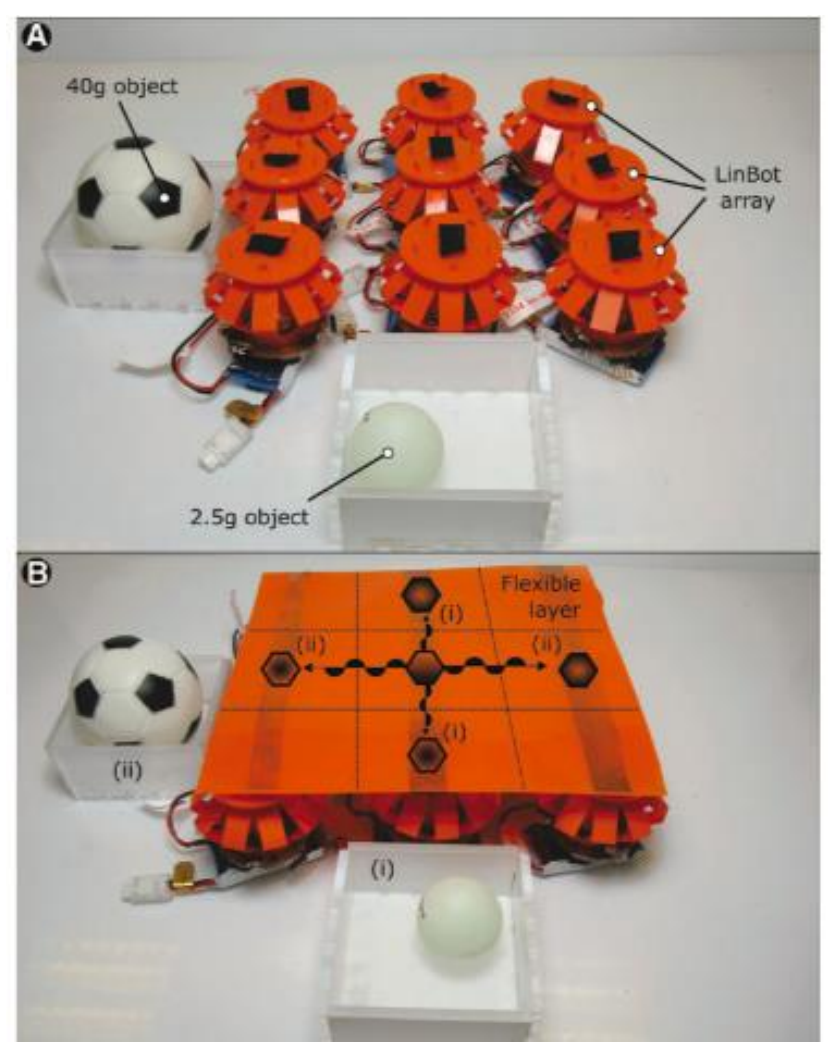

Figure 27. Peristaltic sorter and addressing system. (A) The peristaltic sorter without the flexible layer on top of it, showing the Linbot array. (B) The peristaltic sorter with the flexible layer attached. The behavior of the sorter is shown with the central Linbot detecting the weight of an object and its neighbors actuating to roll the object in the desired direction based on weight ${ }^{[255]}$.

In a similar work, P. Nemtiz et al. presented a modular worm-like robot (Wormbot) ${ }^{[256,257]}$, which utilized voice coils as a new paradigm in soft robot actuation. Drive electronics were incorporated into the actuators, providing a significant improvement in self- sufficiency when compared with existing soft robot actuation modes such as pneumatics or hydraulics (Figure 28). 


\section{WILEY-VCH}

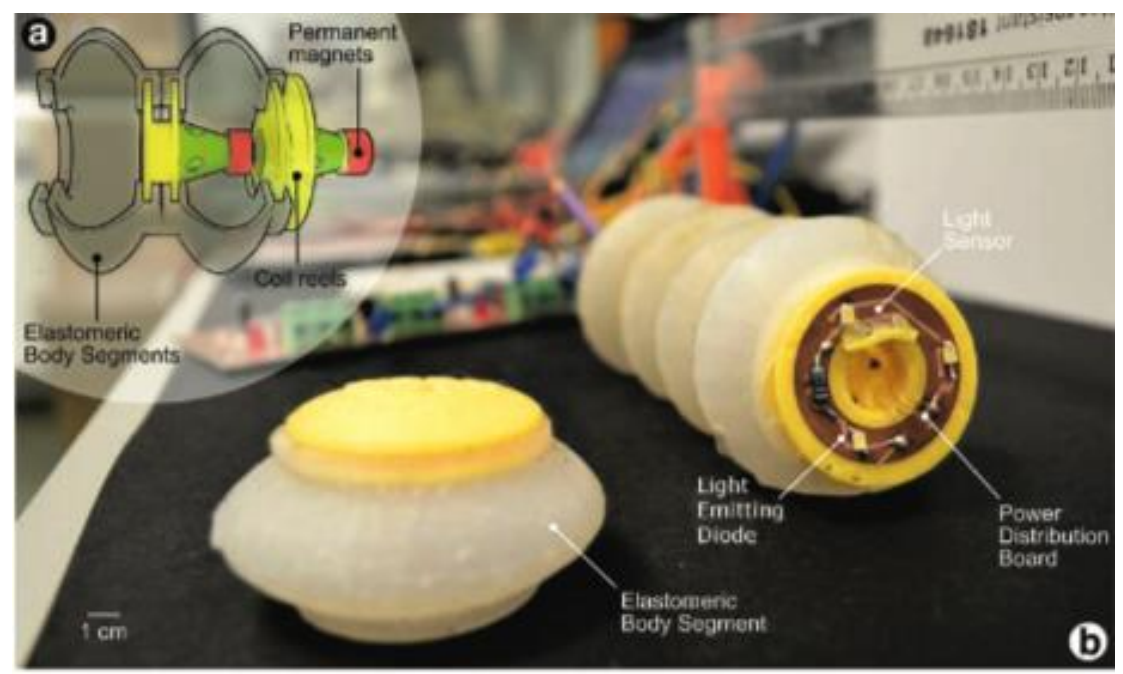

Figure 28. (a) A cutaway sketch of one module from Wormbot, showing the connecting elastomeric body segments and a voice coil actuator. (b) A photograph showing Worm- bot with the rearmost body segment removed to reveal the power distribution board ${ }^{[256]}$.

The body plan of this robot was inspired by the phylum Annelida and consists of three-dimensional printed voice coil actuators, which were connected by flexible silicone membranes. Each electromagnetic actuator engages with its neighbor to compress or extend the membrane of each segment, and the sequence in which they are actuated results in an earthworm-inspired peristaltic motion. They found that a minimum of three segments is required for locomotion, but due to their modular design, robots of any length could be quickly and easily assembled. In addition to actuation, voice coils provided audio input and output capabilities. They demonstrated transmission of data between segments by high-frequency carrier waves and, using a similar mechanism, they noted that the passing of power between coupled coils in neighboring modulesor from an external power source — was also possible. Voice coils are a convenient multifunctional alternative to existing soft robot actuators. Their self- contained nature and ability to communicate with each other are ideal for modular robotics, and the additional functionality of sound input/output and power transfer will become increasingly useful as soft robots begin the transition from early proof-of-concept systems toward fully functional and highly integrated robotic systems. Ebrahimi et.al ${ }^{[244,258]}$. presented another novel highly scalable Electromagnetic Soft Actuator (ESA) based on the principle of solenoids. The actuator was made mostly of silicone rubber so 


\section{WILEY-VCH}

that it can have low stiffness. The major components of the soft actuator included helical coil, soft silicone ferromagnetic core, inner layer, spring linkage and outer layer as shown in Figure 29. The helical coils are made of 100 turn of soft wire. The outer layer of ESA which is included to shield and boost the resultant magnetic field consists of a mixture of $40 \%$ iron oxide and $60 \%$ silicone rubber. This part is included to make a layer of electromagnetic suspension to strengthen the generated field and increase the force.

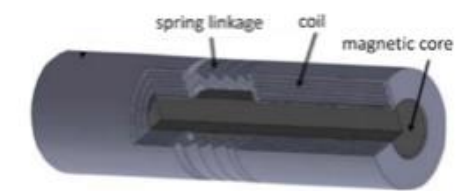

(a)

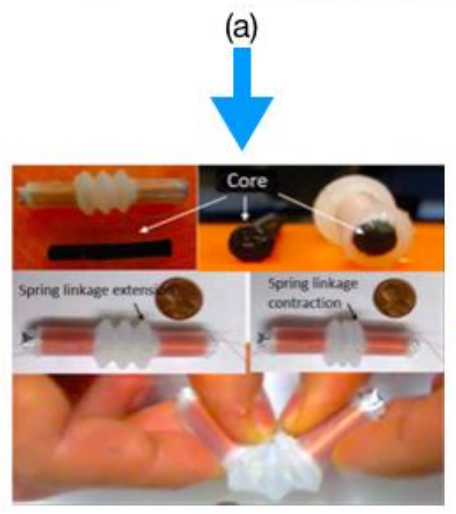

(b)

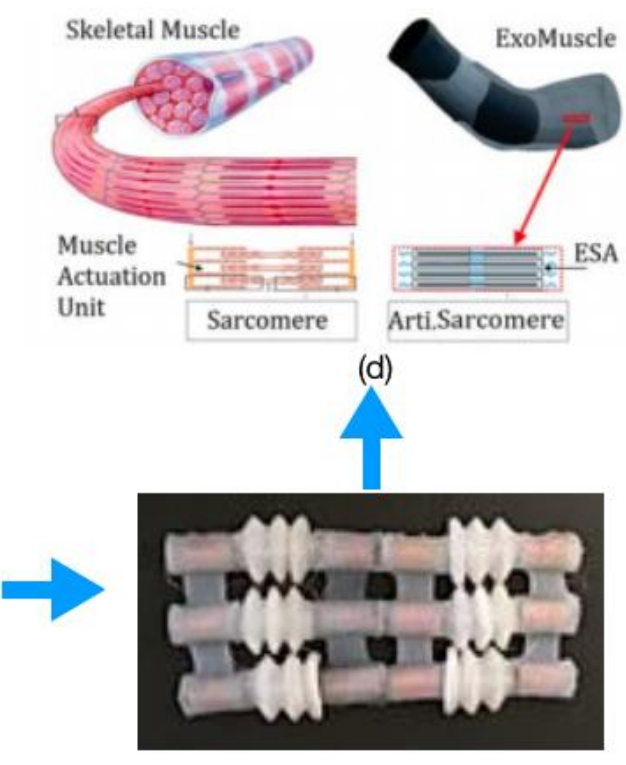

(c)

Figure 29. a) details of an Electromagnetic Soft Actuator ESA, b) mgnetic flexible core and spring linkage connection, c) a 3X2 network of ESA, d) future artificial ExoMuscles based on networked ESA inspired by anatomy of skeletal muscles ${ }^{[244,258]}$.

Interestingly, they found that by scaling down the ESA size, the force/volume ratio increase. Therefore, by miniaturizing the size of the actuator and attaching them as a network, the total resultant force can be greatly enhanced, the same arrangement of linear tiny actuators (actin and myosin filaments) in skeletal muscles ${ }^{[259]}$. The idea is to create an ExoMuscle that can be worn around joints such as elbow or knee to help mobility impaired patients with movement of their limbs.

\section{Magnetically-Guided Capsule Endoscopy [Gastone]}




\section{WILEY-VCH}

Magnetic locomotion of capsule endoscopes has been investigated and proposed in literature through either external permanent magnet or electromagnets. A comprehensive review of magnetic actuation principles and magnetically-driven activation strategies, applied to medical robots and classified by different clinical applications, has been presented by Sliker $e t$ al. in ${ }^{[260]}$, and a recent review, worth to be mentioned, on magnetic methods for remote-manipulation and wirelessactuation tasks in robotics has been presented by Abbott et al. in ${ }^{[261]}$.

In general, if compared to electromagnets, permanent magnetic field sources allow for the generation of a high strength-to-size ratio magnetic field; in other words, given a comparable size and volume, permanent magnets generate lager interaction forces than electromagnets. Another important feature of permanent magnets is related to their intrinsic permanence, i.e. the magnetic field is generated through the material without the need of a power supply, thus offering a wireless non-active magnetic field generator. However, due to possible interference with other equipment in the operating room and interaction with ferromagnetic instrumentation, the latter feature can be considered also in terms of disadvantage since permanent magnets cannot be switched off or controlled in terms of magnetic field direction and strength; shielding is only possible using highpermeability materials to redirect lines of magnetic flux ${ }^{[262]}$. Contrarily, permanent magnets can be easily customized in terms of remanence, dimensions, shapes and magnetization directions, making them suitable for different applications.

Electromagnetic field sources, on the other hand, provide the substantial advantage of the controllability of the generated magnetic field (i.e., from turned on to turned off, modulating the generated field strength and direction), contributing to the safety, flexibility and applicability of the systems in the operating room. However, the main disadvantages are: (1) their high size-tostrength ratio, if compared to permanent magnets; (2) the need of implementing control strategies for modulating, through the supplied current, the electromagnetic field; and (3) the need of a power supply and, frequently, of a cooling system to generate a magnetic field, which usually contributes 


\section{WILEY-VCH}

to a more bulky equipment in the operating room, higher device cost and design complexity, and electrical power demands. Finally, mainly in the case of magnetically-driven locomotion, large electromagnetic sources present a physical limit, since the larger magnetic field that is created along the N-S poles direction can be far from the external surface of the electromagnet, and thus from the medical device if placed parallel to the magnetization direction; this is due to the high number of windings between the centre of the electromagnet and the external surface and to the use of a bulky cooling system.

One of the most noteworthy and a first example of a magnetic-based robotic navigation approach applied to gastrointestinal (GI) endoscopic pill-size robots was first explored between 2008 and 2009 in the framework of a European FP6 project, called "Versatile Endoscopic Capsule for GI TumOr Recognition and therapy (VECTOR project)", coordinated by novineon Healthcare Technology Partners GmbH (Tuebingen, Germany) ${ }^{[263]}$. In the framework of this European project, Ciuti et al. at the Scuola Superiore Sant'Anna (Pisa, Itay) proposed the development of an active locomotion robotic platform based on mutually-interacting permanent magnets, i.e. above the patient body acting as the external magnetic driving source and inside a first prototype of a wireless endoscopic capsule. The developed robotic platform for wireless and wired capsule GI endoscopy combined the benefits of permanent magnetic field strength and limited encumbrance, demonstrating an accurate and reliable control through the use of an external teleoperated anthropomorphic robotic arm and an accelerometer-based localization strategy ${ }^{[264-266]}$.

A similar robotic platform has been developed, since 2011, by the research team lead by Prof. J. Abbott at the University of Utah (Salt Lake City, UT, USA), and it is composed of a 6-DoFs industrial robotic arm with an active-rotatable permanent magnet as the end-effector. The robotic platform has been demonstrated to effectively control and navigate several untethered magnetic devices, e.g. threaded capsule endoscopes and helical microrobots, to operate in natural lumen pathways of the bodies, such as GI, ENT, nervous, and vascular systems ${ }^{[267-271]}$. 


\section{WILEY-VCH}

Apart from the aforementioned multi-purpose robotic platforms for applying magnetic fields for endoscopic capsule locomotion, looking at the research- and industrial-oriented state of the art, it is worth mentioning that magnetic locomotion for capsule endoscopy was mainly applied to the upper (i.e., oesophagus and stomach) and lower (large bowel, mainly) GI tracts. It is worth mentioning that only the most recent and relevant studies will be mentioned in this section of the review paper for the sake of brevity but with the aim of providing a comprehensive overview of the main milestones in this research field; detailed reviews of magnetically-guided capsule endoscopes can be found in ${ }^{[272-274]}$.

Starting with a proof-of-concept developed in 2016, Carpi et al. proposed an external magnetic add-on (i.e., elastic shells made of silicone elastomers mixed with magnetic particles) to provide active magnetic control of a commercially-available wireless capsule endoscope (WCE, M2A Capsule, Given Imaging Ltd., Yoqneam, Israel - today, Medtronic Inc., Minnesota, USA) using hand-held external permanent magnets. Tests, performed in ex-vivo simplified experimental conditions, demonstrated controlled translations, rotations, and roto-translations of the modified WCE $^{[275,276]}$. In 2008, Carpi and Pappone improved the WCE controllability, proposing the use of a commercially-available magnetic robotic platform, originally applied to magnetically-enabled interventional catheter-based cardiovascular procedures, i.e. Stereotaxis Niobe ${ }^{\circledR}$ Robotic Magnetic Navigation System (Stereotaxis Inc., St. Louis, MO, USA), for the active control of the modified commercially-available WCE housing, this time, an external magnetic add-on composed of two solid neodymium-based magnetic semi-cylindrical shells. Effective magnetically-controlled capabilities were experimentally assessed inside a stomach-like cavity into a human-sized plastic phantom under fluoroscopy ${ }^{[277-279]}$. Finally, the same authors demonstrate in 2010 in-vivo accurate robotic steering (omnidirectional steering accuracy of $1^{\circ}$ ) and non-invasive 3D localization (error of $1 \mathrm{~mm}$ ) of the same magnetically-modified commercially-available WCE within each of the 


\section{WILEY-VCH}

main regions of the upper and lower GI tract (oesophagus, stomach, small bowel, and colon) in a domestic pig model ${ }^{[280]}$.

In 2010, Given Imaging Ltd. in collaboration with Prof. Paul Swain, conducted the first in-vivo human magnetic manipulation trial of a modified WCE, i.e. PillCam ${ }^{\mathrm{TM}}$ COLON Capsule embedding neodymium-iron-boron magnets, in the upper GI tract (oesophagus and liquid-filled stomach), through an external hand-held permanent magnet; the study demonstrated the feasibility of the remote manipulation of a modified WCE in humans and larger clinical studies, involving ten healthy participants, were conducted successfully in the oesophagus ${ }^{[281]}$ and stomach ${ }^{[282]}$. A similar research-oriented study, including a hand-held rotary magnet to navigate a magnetic capsule in ex-vivo trials, has been presented by Lien et al. in $2012^{[283]}$. On the other hand, commercially-available hand-held magnetic field-based systems have been developed by the companies Jianshan Science and Technology (Chongqing, China) and Intromedic Ltd. (Seoul, Korea) with the OMOM Controllable Capsule System and MiroCam ${ }^{\circledR}$ Navi MC1000-WM, respectively, demonstrating upper GI tract manoeuvrability in human models ${ }^{[284,285]}$.

In 2010, Siemens Healthcare AG (Erlangen, Germany) and Olympus Medical Corp. (Tokyo, Japan) developed the first electromagnetic capsule manipulation system successfully validated in in-vivo gastric clinical trials, using twelve body-external electromagnetic coils. The proposed MRI-based Siemens Healthcare AG external platform allows 5-DOFs control of a single-use modified Olympus Medical Corp. WCE (31 mm in length and $11 \mathrm{~mm}$ in diameter) embedding a small permanent magnet ${ }^{[286-288]}$. Other significant examples of electromagnetic navigation systems, using modified MRIs or complex electromagnetic-based platforms, for meso- to nanoscale robots, have been proposed by the research teams led by Prof. M. Sitti ${ }^{[289]}$, Prof. S. Martel

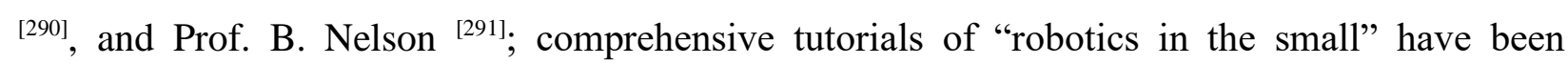
presented in ${ }^{[292,293]}$. 


\section{WILEY-VCH}

A commercially-available platform dedicated to robotic capsule gastroscopy, worth to be mentioned in the section of this review, is the robotic magnetic capsule guidance system produced by Ankon Technologies Co., Ltd. (Wuhan, China). The platform is composed of a 5-DoFs arm that controls an external single spherical permanent magnet able to generate a $200 \mathrm{mT}$ static magnetic field in a $500 \mathrm{~mm}^{3}$ working volume. The generated permanent magnetic field teleoperates an endoscopic pill-size capsule, embedding a small permanent magnet. The platform, patented and clinically approved with CFDA clearance in 2013, has been installed in over hundreds of medical centres in China and successfully clinically validated in humans for gastric examination ${ }^{[294,295]}$. A similar platform, to date at a research/validation phase, has been proposed in 2019 by Cheng et al. and preliminary tested in a cohort of 31 healthy volunteers for gastric examination; the main different is that the examination is conducted in standing than supine position with the aim to improve manoeuvrability in the stomach. The study demonstrated feasibility, safety and satisfactory manoeuvrability of the magnetically-modified WCE in a standing position ${ }^{[296]}$.

In the lower GI tract, i.e. into the colonic district, a first significant example of magnetically-guided endoscopic capsule derived from the previously mentioned European FP6 VECTOR project. Even if the project focused on the developed of magnetically-guided wireless capsule robots, an interesting derivative device of the project consisted of a soft-tethered magnetically-driven capsule for colonoscopy. A proof-of-concept of the robotic colonoscope, presented by Valdastri et al. in $2012^{[297]}$ as a trade-off between capsule and traditional colonoscopy combining the benefits of low-invasive propulsion (through "front-wheel" locomotion) with the multi-functional tether for treatment; was the capsule n.0 and the forerunner of a significant number of derived improvements, new implementations and allied magnetically-guided endoscopic devices. Indeed, the system has been improved in the subsequent years, for instance, in terms of modelling ${ }^{[298,299]}$, tracking and localization ${ }^{[300-302]}$, and control ${ }^{[303-305]}$, towards autonomous locomotion strategies ${ }^{[306]}$ and other applications [307]. In the recent years, a derived novel soft-tethered magnetically-guided 


\section{WILEY-VCH}

colonoscope was designed within a European H2020 project, called "Endoscopic versatile robotic guidance, diagnosis and therapy of magnetic-driven soft-tethered endoluminal robots (Endoo project)", coordinated by Scuola Superiore Sant'Anna (Pisa, Italy) ${ }^{[308]}$. The soft-tethered robotic colonoscope is featured by a high-definition stereoscopic camera with custom-made optics, navigated by an external permanent magnet, precisely controlled by a collaborative anthropomorphic robot (COMAU SpA, Turin, Italy) ${ }^{[309]}$. A noteworthy outcome of the EU project was the development of artificial intelligence algorithms to perform 3D lumen visual reconstruction, vision-based closed-loop control strategies and autonomous detection and measurement algorithms of colonic lesions, e.g. polyps ${ }^{[310-314]}$.

A hand-guided external electromagnetic system for a wireless colonoscope was designed in the framework of a European FP7 project, called "New cost-effective and minimally invasive endoscopic device able to investigate the colonic mucosa, ensuring a high level of navigation accuracy and enhanced diagnostic capabilities (SUPCAM project)", coordinated by S.E.D. Srl (Certaldo, Italy), under the supervision of Dr. Alessandro Tozzi, inventor of the novel capsule spherical concept. The external electromagnetic source, supported by a gravity-compensated arm, navigates, through a generated static magnetic field, a colonoscopic spherical-shape capsule provided with an internal permanent magnet, able to perform a $360^{\circ}$ inspection through inner camera rotation ${ }^{[315-317]}$.

Another significant example of a WCE driven in the colonic tract using electromagnetic fields, in this case alternated, has been presented by Nouda et al. in 2018. A self-propelling capsule endoscope composed by a PillCam ${ }^{\mathrm{TM}}$ SB2 Capsule (Medtronic Inc., Minnesota, USA) modified with a silicone fin and embedding a permanent magnet attached to it $(45 \mathrm{~mm}$ in length and $11 \mathrm{~mm}$ in diameter), has been tested for the first time in a human healthy volunteer. An external platform generates an alternating magnetic field that make the fin shaking and thus propel the capsule with a 3D control. The capsule, inserted in the anus and transported with endoscopic forceps in the 


\section{WILEY-VCH}

descending colon, was able to swim in the lumen in antegrade and retrograde directions without any damage to the mucosa ${ }^{[318]}$.

Examples of the use of magnetic fields, both permanent and electromagnetic, not for locomotion purposes but as activation means for endoluminal treatment or therapy of meso-scale robots have been widely explored and designed in the last years. Being out of the main topic of the section, i.e. magnetically-guided capsule endoscopy, the most significant examples are delegated to this comprehensive review paper published by Sliker et al. in ${ }^{[260]}$; recent and significant examples have been developed in 2020 by Son et al. ${ }^{[319]}$ and Kim et al. ${ }^{[320]}$.

\section{Tetherless Micro-grippers and Magnetic Micromanipulation [Islam]}

Manipulation and assembly of micro-objects has potential unique applications in many areas including biomedicine, chemistry, nanotechnology and biology. These applications have experienced several significant advances through manipulator-based and tetherless micromanipulation. ${ }^{[321,322]}$ Manipulator-based micromanipulation relies on the miniaturization of microelectromechanical systems. At this scale, surface, adhesion, and drag forces have significant influence on the interactions between the micro-objects which makes precise positioning at prescribed locations challenging using manipulator-based techniques. In the case of tetherless micromanipulation, microrobots are used to exert forces via mechanical contact or fluidic trapping without direct contact or use utilize fluid boundary layers to produce contact-free motion. In this technique, the geometric scaling has been enabled through magnetic, ${ }^{[323,324]}$ acoustic, ${ }^{[325]}$ light, ${ }^{[326]}$ and chemical, ${ }^{[327,328]}$ stimuli to transmit power wirelessly. Hence, they can overcome the disadvantages of manipulator-based techniques.

Randhawa et al. have demonstrated pick and place tasks using chemo-mechanically triggered micro-grippers. ${ }^{[329]}$ Their design consists of a trilayer hinge joint capable of opening and closing by residual stresses, and the ability to manipulate tubes and bead has been demonstrated. Since 


\section{WILEY-VCH}

these micro-grippers could be opened and closed by chemicals, their material and range of applications are limited. Leong et al. have developed thermobiochemically actuated microgrippers suitable under biological conditions. ${ }^{[330]}$ These micro-grippers are remotely actuated by a temperature trigger and picking up beads off substrates and removal of cells from tissue samples have been demonstrated. Fusco et al. have also used a similar approach and presented a microrobotic platform that works by increasing the temperature to allow for controlled encapsulation and release of micro-objects. ${ }^{[331]}$ Diller and Sitti have demonstrated threedimensional microassembly and parallel operation by multiple micro-grippers (force- and torquebased micro-grippers) using uniform field and field gradient to achieve programmable magnetic actuation. ${ }^{[332]}$ Zhang et al. have also presented autonomous three dimensional micro-grasping and cargo delivery. ${ }^{[333]}$ Microassembly of micro-objects to microstructures have also been demonstrated using cluster of paramagnetic microparticles under the influence of controlled magnetic field gradients by Khalil et al. ${ }^{[334]}$ In the previous techniques, the micro-grippers exert manipulative forces via direct contact with the micro-objects, and adhesive forces are likely to prevent their release at prescribed positions.

This problem could be partially overcome if an external stimulus can influence the interaction between the microrobot and the micro-object. Consider, for example, the soft microrobot illustrated in Figure 30. It consists of a magnetic head and an ultra-thin flexible tail. ${ }^{[335]}$ The microrobot undergoes travelling-wave propulsion under the influence of uniform magnetic field along the desired direction of motion with a sinusoidally varying orthogonal component, leading to controlled swim toward the micro-objects (microbeads). At $t=83$ seconds, the soft microrobot achieves contact manipulation of the micro-object toward the prescribed position (red mark). Once positioned at the target position, the microrobot has to swim away from the manipulation site. Therefore, the external stimulus, in this case we have magnetic actuation, has to enable the microrobot to break free from the micro-obeject using relatively high actuation frequencies. Figure 


\section{WILEY-VCH}

30 shows that the microrobot ( $t=506$ seconds) swims away from the manipulation site after a successful release of the micro-object. However, the adhesive forces between the microrobot and the micro-object result in stickiness, which can be avoided in all times in the case of non-contact manipulation.

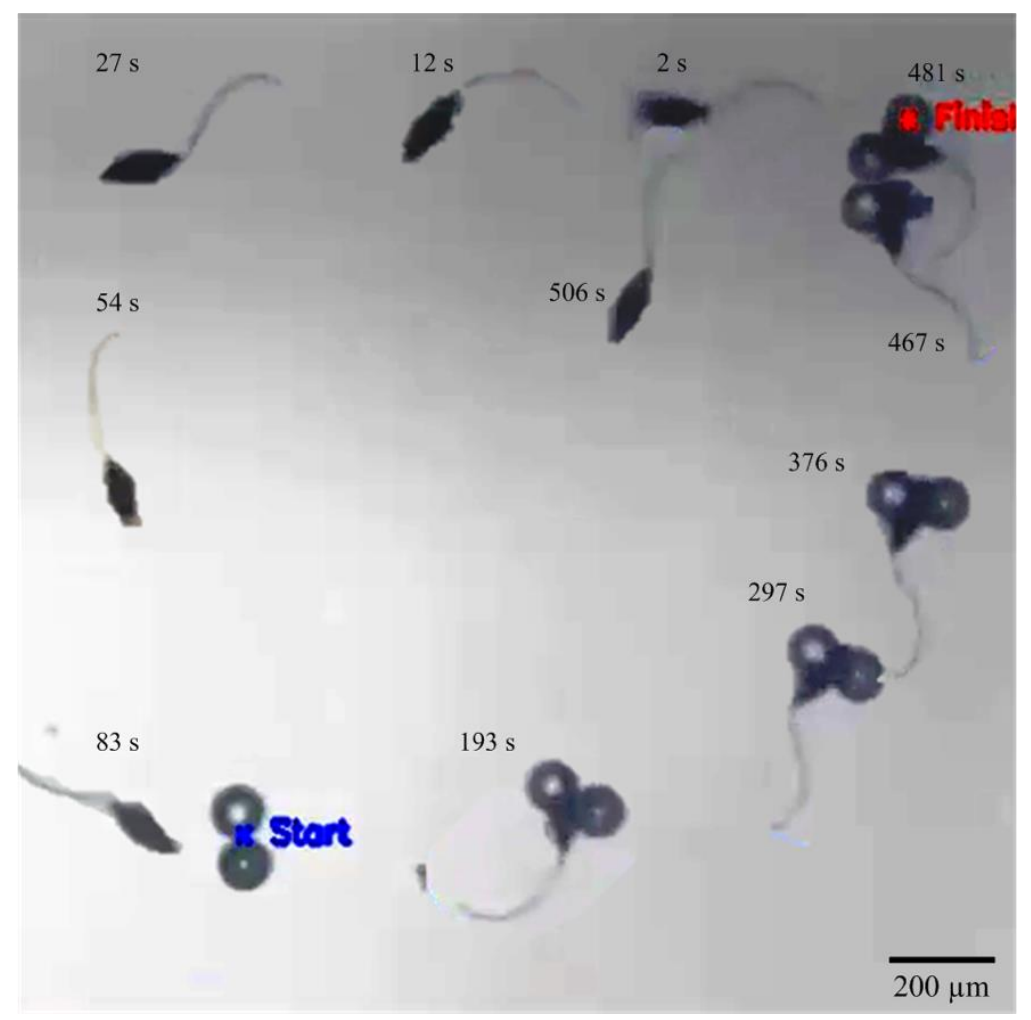

Figure 30. A soft microrobot translates two non-magnetic polystyrene microbeads by direct contact toward a reference position (red mark). At $t=2$ seconds, the microrobot swims toward the microbeads (blue mark) using uniform magnetic field along the desired direction of motion with sinusoidally varying orthogonal components. ${ }^{[335]}$ Images obtained during 506 seconds and superimposed.

Non-contact micromanipulation techniques are more suitable when direct contact results in contamination to biological samples or stickiness, and provide alternative approach for micrograsping. These techniques include electrophoresis, ${ }^{[336]}$ optical trapping, ${ }^{[337]}$ fluidic trapping, ${ }^{[338]}$ acoustic manipulation, ${ }^{[339]}$ vibration, ${ }^{[340,341]}$ and magnetic manipulation. ${ }^{[342]}$ Most of these systems have limited workspace with the exception of magnetic manipulation. Floyed et al. have presented a contact-free micromanipulation method based on fluidic trapping using microrobots. ${ }^{[343,344]}$ These microrobots are utilized to create local flow to push microspheres, and two methods of non- 


\section{WILEY-VCH}

contact manipulation based on front and side pushing have been presented. In this method, stickslip motion of the magnetic microrobots under the influence of magnetic fields are used to create the fluid flow. This approach depends on a nearby surface to achieve the stick-slip motion. Peyer et al. have also shown fluidic trapping using artificial bacterial flagella under the influence of rotating magnetic fields ${ }^{[345]}$ The rotation of these helical microrobots create rotational fluid flow and manipulate microbeads without contact. Petit et al. have also demonstrated selective trapping and micromanipulation of microobjects using a tangential flow-field induced by a rotating nanowire. ${ }^{[346]}$ They have demonstrated sequential pick-and-place micromanipulation of polystyrene microspheres with a microvortex created by the rotating nanowire. This method has been also implemented to manipulate (fluidic trapping and transportation) individual E. coli bacterium with the controlled microvortex near a solid boundary. This technique overcomes the limitation associated with optical tweezers, magnetic tweezers, dielectrophoresis which cannot be used for the manipulation of many biological samples.

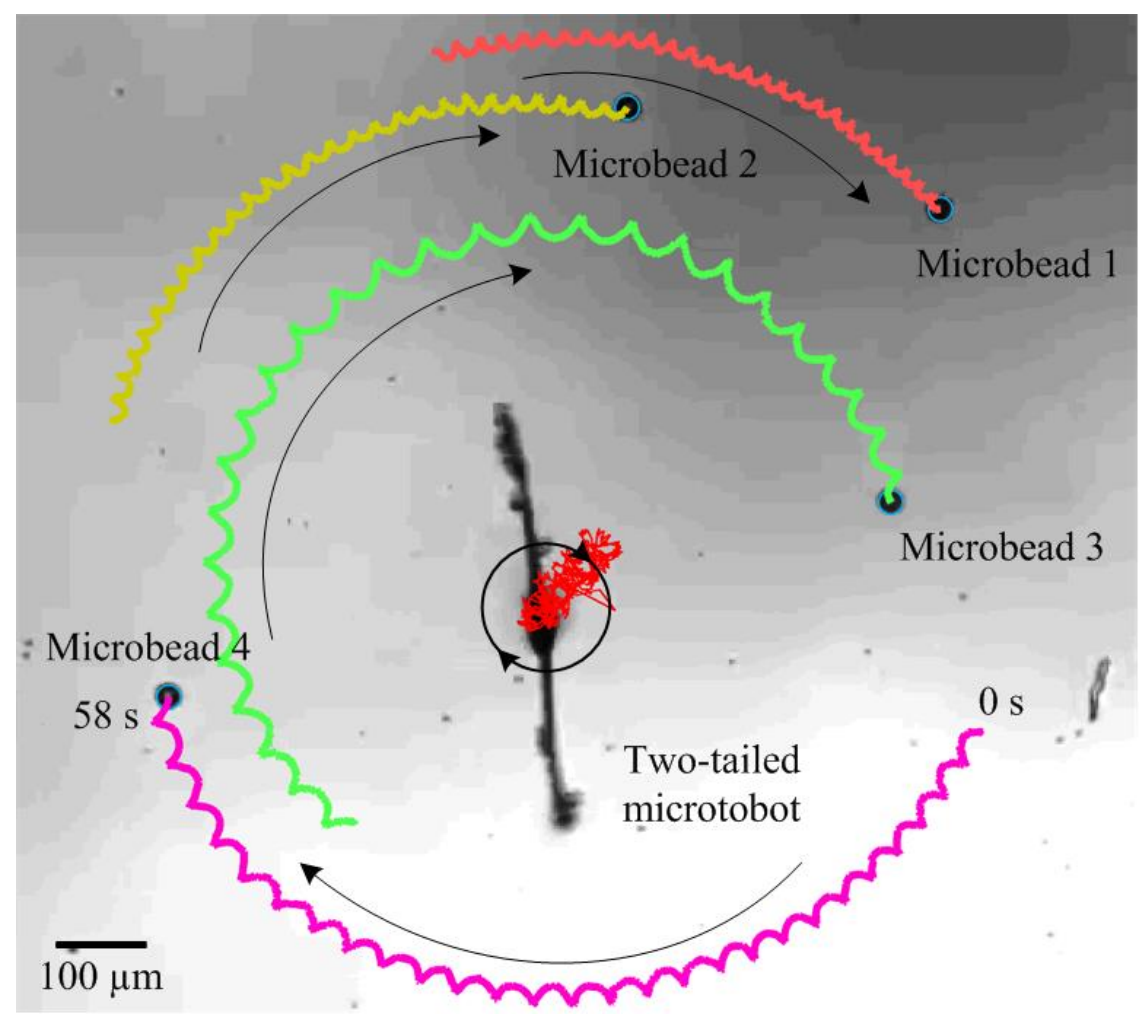




\section{WILEY-VCH}

Figure 31. A two-tailed soft microrobot rotates under the influence of a rotating magnetic field. The induced flow-field enables non-magnetic polystyrene microbeads to orbit the soft microrobot along sprocket-like trajectories at different angular velocities. The non-contact manipulation has significant importance in the manipulation of biological samples. ${ }^{[347]}$ Images obtained during 58 seconds and superimposed. The red line indicates the trajectory of the center of rotation of the twotailed microrobot, and the black arrows indicate the direction of rotation.

Ye et al. have also used the locally-induced rotational fluid flows to achieve non-contact micromanipulation. ${ }^{[348]}$ This method relies on the velocity field which decay in space nearly as $1 / r^{2}$, where $r$ is the distance between the rotating microrobot and the micro-object, as shown in

Figure 31. Ye et al. have utilized groups of untethered magnetic microrobots that can have reconfigurable configurations to create virtual fluidic channels. The microrobots can be arranged in any desirable configuration to create the desired flow-field in the channel. Non-contact pushing and pulling of non-magnetic micro-objects have also been achieved by microrobots under the influence of controlled magnetic field gradient, ${ }^{[349]}$ and scaled bilateral tele-manipulation enabled the operator to sense the interaction forces between the microrobot and the micro-objects. ${ }^{[347]}$ Rolling microrobots have also been used by Tung et al. to manipulate micro-objects. ${ }^{[350]}$ Flowfields are created by transversely magnetized rolling microrobots and micromanipulation is achieved through the transmitted forces to the manipulated micro-objects. The capability of soft microrobots to swim controllably toward the manipulation site to achieve non-contact manipulation, and swim away after manipulation has been demonstrated by Khalil et al. ${ }^{[351]}$ In this approach, two tailed soft microrobots achieve a combination of flagellar swim and rotations to move toward micro-objects and create controlled flow-field, respectively. This induced flow-field enables the micro-objects to orbit the soft microrobot (without contact) and translate to prescribed positions in open- and closed-loop. They have shown that the additional soft tail increases the angular velocity of the orbiting microbeads and results in a sprocket-like trajectory, as shown in Figure 31. They have also shown that the unique geometry of the soft microrobot enables bidirectional motion. This feature has a significance importance to swim away from the manipulation 


\section{WILEY-VCH}

site without affecting the positioning accuracy of the micro-object at the prescribed position. Soft microrobots with travelling-wave propulsion have a wave-pattern that can be scale with the actuation frequency. At relatively low actuation frequencies, the overall amplitude of the pattern is relatively high, which is a desirable feature during non-contact manipulation and fluidic trapping. ${ }^{[352]}$ This amplitude decreases at relatively high actuation frequencies and creates lower flow-field in the background fluid, which is desirable to move away from the manipulation site. In addition, soft microrobots have the advantage that they do not require a nearby surface for locomotion. Rigid microrobots are dependent on stick-slip motion or rolling, thereby limiting the manipulation site to regions near to a solid boundary. In contrast to rigid microrobots, soft microrobots (or microrobots based on travelling-wave propulsion) can achieve non-contact and contact manipulation everywhere in the fluid. Consider, for example, artificial bacterial flagella, ${ }^{[345]}$ which have rigid helical structure and can swim without in $3 \mathrm{D}$ space and does not depend on a nearby solid boundary. These microrobots can achieve fluidic trapping and transportation of micro-objects and biological samples. However, they achieve fluidic trapping through rigid body rotation and it is likely that the positioning accuracy is decreased when the microrobot swim away from the manipulation site. In contrast, soft microrobots have wave-pattern that scales with the actuation frequencies. Therefore, they can achieve 3D contact and non-contact manipulation without affecting the positioning accuracy between several tasks. These desirable features, combined to their simplicity in design and manufacturing, less density, and high level of biodegradability, ${ }^{[353]}$ provide soft microrobots with relative importance in manipulation of microobject and significant importance in manipulation of biological samples. ${ }^{[346]}$

\section{Magnetically Actuated Steerable Catheters [Iacovacci]}

Magnetic actuation has attracted increasing attention in those application fields where dimensional constraints significantly hamper the employment of standard actuation technologies. In this regard, the fields of surgical robotics, medical devices, interventional systems and microrobotics have 


\section{WILEY-VCH}

greatly benefitted from the use of magnetic fields for wireless actuation, either steering or locomotion. Reducing invasiveness and pursuing direct target reaching to deliver the therapy in a more efficient way while reducing side effects is one of the challenges in medical robotics and interventional procedures.

The current best practice in interventional vascular procedures is represented by manual catheter control (catheter push and pull to enable target reaching) and time-consuming interventional procedures typically guided by $\mathrm{x}$-ray imaging. Whilst catheters insertion can be performed through dedicated cable-driven advancing systems, finely controlling their bending and the force exerted on vessels wall is extremely challenging and typically performed manually.

Wireless magnetic actuation can be particularly beneficial for catheter steering in light of the complex and tortuous nature of the vascular tree and of the small vessels caliber. Furthermore, the possibility to exert a stable force on a magnetic component can be particularly useful to overcome the wide fluctuations experienced in manual catheters when in contact with moving tissues e.g. the beating heart in applications such as radiofrequency ablation.

Significant advances have been recently made in magnetic navigation systems and magnetically steerable catheters/guidewires, showing potential benefits such as reduced radiation doses and improved access to hard-to-reach and tortuous anatomies. ${ }^{[354]}$

In order to enable catheter remote magnetic control, it is necessary to establish a magnetic link between an external magnetic field source (a set of magnets or electromagnets) and a magnetic element mounted on the catheter. When a magnetic object is exposed to a magnetic field, it can experience a certain magnetic force $\mathrm{F}_{\mathrm{m}}$ and torque $\mathrm{T}_{\mathrm{m}}$ that can be expressed as follows in Equation 4 and 5:

$$
\begin{aligned}
& F_{m}=\int_{V} \quad(m \cdot \nabla) B d V \\
& T_{m}=\int_{V} \quad(m \times \nabla) B d V
\end{aligned}
$$




\section{WILEY-VCH}

where $\mathrm{m}$ is the catheter magnetization, $\mathrm{V}$ is the volume of the magnetic element included in the catheter and B is the magnetic flux density. ${ }^{[355]}$ In order to enable establishing the aforementioned magnetic link, endovascular catheters and guidewires both including ferromagnetic components, permanent magnets, coils or based on innovative magnetic materials have been reported both as commercial and research systems. ${ }^{[356]}$ We will analyze in the following all these solutions by reviewing the most significant examples reported in the state of the art.

The inclusion of one or more small permanent magnets on the catheter tip to enable tip steering and controlled vessel contact is undoubtedly the most straightforward solution and the strategy adopted by many research groups and companies. Biosense Webster commercializes a $8 \mathrm{~F}$ magnetic endocardial catheter for atrial fibrillation ablation $\left(\mathrm{CARTO}^{\circledR} \mathrm{THERMOCOOL}^{\circledR} \mathrm{RMT}\right)$ suitable for navigation with the Niobe (Stereotaxis) system, a commercial magnetic navigation system based on two rotating strong permanent magnets. Consisting in a flexible body, the catheter includes a magnetic tip and multiple radiofrequency electrodes for ablation. A small magnet embedded in the catheter tip causes the catheter to align and to be steered by the external magnetic field whereas a motor drive advances or retracts the catheter, enabling complete remote navigation. Similarly, a $0.36 \mathrm{~mm}$ diameter magnetic guidewire (Cronus, Stereotaxis) suitable for navigation with the Stereotaxis system was proposed. The guidewire includes a coiled distal segment to which a gold-encapsulated NdFeB magnet is attached to enable guidewire steering. ${ }^{[357]}$ Choi and coworkers extended this concept by proposing a soft microrobotic system to be mounted on the tip of conventional guidewire to increase their steerability. The microrobot is fabricated via replica molding and features a soft body made of polydimethylsiloxane, two permanent magnets, and a microspring for an overall $500 \mu \mathrm{m}$ diameter. The angulation of the microrobot can be controlled from $21.1^{\circ}$ to $132.7^{\circ}$ by using a magnetic field of an intensity of $15 \mathrm{mT}$. ${ }^{[358]}$ Permanent magnets have also been employed in combination with smart materials to enable stiffness control. Nelson and co-workers recently reported about a variable stiffness multiple segments magnetic catheter 


\section{WILEY-VCH}

combining a permanent magnet placed on the catheter tip and a low melting point alloy allowing to independently change the stiffness of each module through electrical currents. The resulting system matches the precision of magnetic navigation with additional degrees of freedom provided segments stiffness variations. The catheter has a $2.33 \mathrm{~mm}$ diameter and includes a working channel for additional tools insertion. ${ }^{[359]}$

A different approach to be pursued while trying to provide a catheter or a guidewire with magnetic properties is to include ferromagnetic components. However, this kind of approach calls for two different magnetic excitation fields to accomplish catheter orientation and steering: a permanent magnetic field to magnetize the ferromagnetic element to its saturation and a magnetic gradient to produce deflections.

MRI systems combining strong static fields and additional gradients have been successfully employed in this kind of application together with catheters and guidewires equipped with ferromagnetic beads on the tip. This approach was mainly pursued by Martel and co-workers by including one or more ferromagnetic beads either on catheters, ${ }^{[360,361]}$ or guidewire tips, ${ }^{[362]}$ to produce controlled steering in a clinical MRI system. Feasibility of such approach was demonstrated in vivo on swine model. Particularly interesting is the recent work by Martel and coworkers who used the fringe field of clinical MRI to combine catheter push and pull in order to better counteract friction forces and enable better steering also in small diameter vessels. ${ }^{[363]}$ MRIbased navigation was proposed also in combination with clinical-grade microcatheters with a solenoid coil at the distal tip. ${ }^{[364]}$ In this case, the local magnetic field produced by the tip coil interacts with the main field of the MRI system thus steering the catheter. However, heating issues with consequent blood vessels damage risks, are associated with such strategy.

Last but not least, innovative flexible magnetic materials have been employed in endovascular applications to cope with the bottleneck of size imposed by permanent magnets manufacturing. Zhao and co-workers presented a submillimeter-scale, self-lubricating soft continuum robot with 


\section{WILEY-VCH}

omnidirectional steering and navigating capabilities based on magnetic actuation, which are enabled by programming ferromagnetic domains in its soft body while growing hydrogel skin on its surface. In particular, a composite polymeric matrix based on polydimethylsiloxane and silicacoated $\mathrm{NdFeB}$ particles was permanently magnetized and printed in the desired shape while selectively orienting the pre-magnetized particles to accomplish the desired magnetization pattern. The obtained magnetic guidewire was then provided with an hydrogel skin to reduce navigation friction. ${ }^{[365]}$

Most of the catheter and guidewire systems reviewed in this section were aimed at interventional procedures such as ablation. However, smart permanent magnets-based catheters have been recently proposed also for liquid biopsy and for enhancing the safety of micro and nanorobots based therapeutic paradigms. Vermesh et al. reported the development of a flexible magnetic wire to be inserted and removed through a standard intravenous catheter to capture biomarkers that have been previously labelled with injected magnetic particles. The MagWIRE's consists in a simple string of 60 cylindrical NdFeB magnets (N50-grade, $0.75 \mathrm{~mm}$ in diameter by $1 \mathrm{~mm}$ in length) encapsulated within a Polytetrafluoroethylene (PTFE) tubing with an inner diameter of $0.81 \mathrm{~mm}$ and wall thickness of $38.1 \mu \mathrm{m}$. This kind of magnetic arrangement enables to produce a large magnetic field gradient and attractive force thus to maximize specific biomarkers capture. ${ }^{[366]}$

Iacovacci et al. extended the concept of magnetic micro-objects capture to the field of micro/nanorobotics and nanomedicine with the aim to increase the safety of such therapeutic procedures. To become fully acceptable in fact medical microrobots should be either biodegradable or removed after task execution, to not raise short- and long-term side effects. To this aim an intravascular catheter able to efficiently retrieve from the bloodstream magnetic micro and nanorobots was proposed. The device consists of a miniature module, based on 27 permanent magnets arranged in two coaxial series, integrated into a clinically used 12 French catheter provided with a tip balloon for blood canalization within the retrieval catheter. This device can 


\section{WILEY-VCH}

capture $\approx 94 \%$ and $78 \%$ of the unused agents when using as carriers 500 and $250 \mathrm{~nm}$ nominal diameter superparamagnetic iron oxide nanoparticles, respectively. ${ }^{[367,368]}$

\section{References}

[1] L. Sliker, G. Ciuti, M. Rentschler, A. Menciassi, Expert Rev Med Devices 2015, 12, 737.

[2] V. Moura, Algarve, Portugal, 2012; p. 48.

[3] F. Schmitt, O. Piccin, L. Barbé, B. Bayle, Front. Robot. AI 2018, 5.

[4] J. Sikorski, I. Dawson, A. Denasi, E. Hekman, S. Misra, Singapore, 2017.

[5] N. Garbin, C. Di Natali, J. Buzzi, E. De Momi, P. Valdastri, Journal of Medical Devices 2015, 9.

[6] M. Rea, D. McRobbie, H. Elhawary, Z. T. H. Tse, M. LampÉrth, I. Young, IEEE/ASME Transactions on Mechatronics 2008, 13, 379.

[7] H. C. Berg, R. A. Anderson, Nature 1973, 245, 380.

[8] R. Dreyfus, J. Baudry, M. L. Roper, M. Fermigier, H. A. Stone, J. Bibette, Nature 2005, 437, 862.

[9] M. Roper, R. Dreyfus, J. Baudry, M. Fermigier, J. Bibette, H. A. Stone, Journal of Fluid Mechanics 2006, 554, 167.

[10] W. Gao, S. Sattayasamitsathit, K. M. Manesh, D. Weihs, J. Wang, J. Am. Chem. Soc. 2010, 132, 14403.

[11] O. S. Pak, W. Gao, J. Wang, E. Lauga, Soft Matter 2011, 7, 8169.

[12] E. Diller, J. Zhuang, G. Zhan Lum, M. R. Edwards, M. Sitti, Appl. Phys. Lett. 2014, 104, 174101.

[13] B. Jang, E. Gutman, N. Stucki, B. F. Seitz, P. D. Wendel-García, T. Newton, J. Pokki, O. Ergeneman, S. Pané, Y. Or, B. J. Nelson, Nano Lett. 2015, 15, 4829.

[14] T. Li, J. Li, H. Zhang, X. Chang, W. Song, Y. Hu, G. Shao, E. Sandraz, G. Zhang, L. Li, J. Wang, Small 2016, 12, 6098.

[15] D. J. Bell, S. Leutenegger, K. M. Hammar, L. X. Dong, B. J. Nelson, In Proceedings 2007 IEEE International Conference on Robotics and Automation; 2007; pp. 1128-1133.

[16] L. Zhang, J. J. Abbott, L. Dong, B. E. Kratochvil, D. Bell, B. J. Nelson, Appl. Phys. Lett. 2009, 94, 064107.

[17] A. Ghosh, P. Fischer, Nano Lett. 2009, 9, 2243.

[18] I. S. M. Khalil, A. Fatih Tabak, A. Klingner, M. Sitti, Appl. Phys. Lett. 2016, 109, 033701.

[19] J. Li, S. Sattayasamitsathit, R. Dong, W. Gao, R. Tam, X. Feng, S. Ai, J. Wang, Nanoscale 2014, 6, 9415.

[20] C. C. J. Alcântara, S. Kim, S. Lee, B. Jang, P. Thakolkaran, J.-Y. Kim, H. Choi, B. J. Nelson, S. Pané, Small 2019, 15, 1970086.

[21] Z. Ye, S. Régnier, M. Sitti, IEEE Transactions on Robotics 2014, 30, 3.

[22] D. Schamel, A. G. Mark, J. G. Gibbs, C. Miksch, K. I. Morozov, A. M. Leshansky, P. Fischer, ACS Nano 2014, 8, 8794.

[23] S. Schuerle, S. Pané, E. Pellicer, J. Sort, M. D. Baró, B. J. Nelson, Small 2012, 8, 1498.

[24] X. Yan, Q. Zhou, J. Yu, T. Xu, Y. Deng, T. Tang, Q. Feng, L. Bian, Y. Zhang, A. Ferreira, L. Zhang, Advanced Functional Materials 2015, 25, 5333.

[25] S. Tottori, L. Zhang, F. Qiu, K. K. Krawczyk, A. Franco-Obregón, B. J. Nelson, Adv. Mater. Weinheim 2012, 24, 811.

[26] K. Kobayashi, K. Ikuta, Appl. Phys. Lett. 2008, 92, 262505.

[27] A. Barbot, D. Decanini, G. Hwang, Sci Rep 2016, 6, 1.

[28] P. Satir, T. Heuser, W. S. Sale, Bioscience 2014, 64, 1073.

[29] J. R. Blake, M. A. Sleigh, Biol Rev Camb Philos Soc 1974, 49, 85.

[30] B. A. Evans, A. R. Shields, R. L. Carroll, S. Washburn, M. R. Falvo, R. Superfine, Nano Lett. 2007, 7, 1428. 


\section{WILEY-VCH}

[31] A. R. Shields, B. L. Fiser, B. A. Evans, M. R. Falvo, S. Washburn, R. Superfine, PNAS 2010, 107, 15670.

[32] S. Kim, S. Lee, J. Lee, B. J. Nelson, L. Zhang, H. Choi, Sci Rep 2016, 6, 1.

[33] S. N. Khaderi, M. G. H. M. Baltussen, P. D. Anderson, D. Ioan, J. M. J. den Toonder, P. R. Onck, Phys. Rev. E 2009, 79, 046304.

[34] F. Meng, D. Matsunaga, J. M. Yeomans, R. Golestanian, Soft Matter 2019, 15, 3864.

[35] M. Medina-Sánchez, L. Schwarz, A. K. Meyer, F. Hebenstreit, O. G. Schmidt, Nano Lett. 2016, 16, 555.

[36] F. Qiu, R. Mhanna, L. Zhang, Y. Ding, S. Fujita, B. J. Nelson, Sensors and Actuators B: Chemical 2014, 196, 676.

[37] S. Jeon, S. Kim, S. Ha, S. Lee, E. Kim, S. Y. Kim, S. H. Park, J. H. Jeon, S. W. Kim, C. Moon, B. J. Nelson, J. Kim, S.-W. Yu, H. Choi, Science Robotics 2019, 4.

[38] C. Xin, L. Yang, J. Li, Y. Hu, D. Qian, S. Fan, K. Hu, Z. Cai, H. Wu, D. Wang, D. Wu, J. Chu, Adv. Mater. Weinheim 2019, 31, e1808226.

[39] X. Wang, X.-H. Qin, C. Hu, A. Terzopoulou, X.-Z. Chen, T.-Y. Huang, K. Maniura-Weber, S. Pané, B. J. Nelson, Advanced Functional Materials 2018, 28, 1804107.

[40] A. Servant, F. Qiu, M. Mazza, K. Kostarelos, B. J. Nelson, Adv. Mater. Weinheim 2015, 27, 2981.

[41] D. Gong, J. Cai, N. Celi, L. Feng, Y. Jiang, D. Zhang, Journal of Magnetism and Magnetic Materials 2018, 468, 148.

[42] T. Xu, J. Yu, X. Yan, H. Choi, L. Zhang, Micromachines 2015, 6, 1346.

[43] B. R. Donald, C. G. Levey, C. D. McGray, I. Paprotny, D. Rus, Journal of Microelectromechanical Systems 2006, 15, 1.

[44] O. Ohmichi, Y. Yamagata, T. Higuchi, Journal of Microelectromechanical Systems 1997, 6, 200.

[45] D. R. Frutiger, K. Vollmers, B. E. Kratochvil, B. J. Nelson, The International Journal of Robotics Research 2009.

[46] C. Pawashe, S. Floyd, M. Sitti, The International Journal of Robotics Research 2009.

[47] I. A. Ivan, G. Hwang, J. Agnus, M. Rakotondrabe, N. Chaillet, S. Régnier, In 2011 IEEE International Conference on Robotics and Automation; 2011; pp. 102-108.

[48] H.-W. Tung, M. Maffioli, D. R. Frutiger, K. M. Sivaraman, S. Pané, B. J. Nelson, IEEE Transactions on Robotics 2014, 30, 26.

[49] C. Pawashe, S. Floyd, E. Diller, M. Sitti, IEEE Transactions on Robotics 2012, 28, 467.

[50] E. Diller, C. Pawashe, S. Floyd, M. Sitti, The International Journal of Robotics Research 2011.

[51] S. Floyd, E. Diller, C. Pawashe, M. Sitti, The International Journal of Robotics Research 2011.

[52] M. P. Kummer, J. J. Abbott, B. E. Kratochvil, R. Borer, A. Sengul, B. J. Nelson, IEEE Transactions on Robotics 2010, 26, 1006.

[53] E. Diller, J. Giltinan, M. Sitti, The International Journal of Robotics Research 2013.

[54] E. Diller, M. Sitti, ROB 2013, 2, 143.

[55] H. Ceylan, J. Giltinan, K. Kozielski, M. Sitti, Lab Chip 2017, 17, 1705.

[56] B. Hemes, D. Canelon, J. Dancs, N. Papanikolopoulos, In 2011 IEEE International Conference on Robotics and Automation; 2011; pp. 5063-5069.

[57] G.-L. Jiang, Y.-H. Guu, C.-N. Lu, P.-K. Li, H.-M. Shen, L.-S. Lee, J. A. Yeh, M. T.-K. Hou, J. Micromech. Microeng. 2010, 20, 085042.

[58] L. Zhang, T. Petit, Y. Lu, B. E. Kratochvil, K. E. Peyer, R. Pei, J. Lou, B. J. Nelson, ACS Nano 2010, 4, 6228.

[59] J. Burdick, R. Laocharoensuk, P. M. Wheat, J. D. Posner, J. Wang, J. Am. Chem. Soc. 2008, 130, 8164.

[60] S. Sundararajan, P. E. Lammert, A. W. Zudans, V. H. Crespi, A. Sen, Nano Lett. 2008, 8, 1271.

[61] L. O. Mair, S. Chowdhury, G. A. Paredes-Juarez, M. Guix, C. Bi, B. Johnson, B. W. English, S. Jafari, J. Baker-McKee, J. Watson-Daniels, O. Hale, P. Stepanov, D. Sun, Z. Baker, C. Ropp, S. 


\section{WILEY-VCH}

B. Raval, D. R. Arifin, J. W. M. Bulte, I. N. Weinberg, B. A. Evans, D. J. Cappelleri, Micromachines 2019, 10, 230.

[62] C. E. Sing, L. Schmid, M. F. Schneider, T. Franke, A. Alexander-Katz, Proc. Natl. Acad. Sci. U.S.A. 2010, 107, 535.

[63] H. Morimoto, T. Ukai, Y. Nagaoka, N. Grobert, T. Maekawa, Phys Rev E Stat Nonlin Soft Matter Phys 2008, 78, 021403.

[64] P. Tierno, R. Golestanian, I. Pagonabarraga, F. Sagués, Phys. Rev. Lett. 2008, 101, 218304.

[65] Z. Ye, E. Diller, M. Sitti, Journal of Applied Physics 2012, 112, 064912.

[66] H.-W. Tung, K. E. Peyer, D. F. Sargent, B. J. Nelson, Appl. Phys. Lett. 2013, 103, 114101.

[67] R. S. Pieters, H.-W. Tung, D. F. Sargent, B. J. Nelson, IFAC Proceedings Volumes 2014, 47, 7480.

[68] D. Sargent, H.-W. Tung, R. Pieters, B. Nelson, Acta Crystallogr A Found Adv 2014, 70, C1753.

[69] H.-W. Tung, D. F. Sargent, B. J. Nelson, J Appl Cryst 2014, 47, 692.

[70] S. Charreyron, R. S. Pieters, H.-W. Tung, M. Gonzenbach, B. J. Nelson, In 2015 IEEE/RSJ International Conference on Intelligent Robots and Systems (IROS); 2015; pp. 177-182.

[71] W. Jing, N. Pagano, D. J. Cappelleri, In 2013 IEEE International Conference on Robotics and Automation; 2013; pp. 5514-5519.

[72] W. Jing, N. Pagano, D. J. Cappelleri, J Micro-Bio Robot 2013, 8, 1.

[73] M. T. Hou, H.-M. Shen, G.-L. Jiang, C.-N. Lu, I.-J. Hsu, J. A. Yeh, Appl. Phys. Lett. 2010, 96, 024102.

[74] C. Bi, M. Guix, B. V. Johnson, W. Jing, D. J. Cappelleri, Micromachines 2018, 9, 68.

[75] J. Xie, C. Bi, D. J. Cappelleri, N. Chakraborty, American Society of Mechanical Engineers Digital Collection, 2019.

[76] C. Bi, E. E. Niedert, G. Adam, E. Lambert, L. Solorio, C. J. Goergen, D. J. Cappelleri, In 2019 International Conference on Manipulation, Automation and Robotics at Small Scales (MARSS); 2019; pp. 1-6.

[77] Hen-Wei Huang, M. S. Sakar, K. Riederer, N. Shamsudhin, A. Petruska, S. Pané, B. J. Nelson, In 2016 IEEE International Conference on Robotics and Automation (ICRA); 2016; pp. 1719-1724.

[78] W. Hu, G. Z. Lum, M. Mastrangeli, M. Sitti, Nature 2018, 554, 81.

[79] D. Faivre, D. Schüler, Chemical Reviews 2008, 108, 4875.

[80] C. T. Lefèvre, F. Abreu, U. Lins, D. A. Bazylinski, In Metal Nanoparticles in Microbiology; Rai, M.; Duran, N., Eds.; Springer Berlin Heidelberg: Berlin, Heidelberg, 2011; pp. 75-102.

[81] L. Yan, H. Da, S. Zhang, V. M. López, W. Wang, Microbiological Research 2017, $203,19$.

[82] C. T. Lefevre, D. A. Bazylinski, Microbiology and Molecular Biology Reviews 2013, 77, 497.

[83] C. T. Lefèvre, M. Bennet, L. Landau, P. Vach, D. Pignol, D. A. Bazylinski, R. B. Frankel, S. Klumpp, D. Faivre, Biophysical Journal 2014, 107, 527.

[84] O. Felfoul, M. Mohammadi, S. Taherkhani, D. de Lanauze, Y. Zhong Xu, D. Loghin, S. Essa, S. Jancik, D. Houle, M. Lafleur, L. Gaboury, M. Tabrizian, N. Kaou, M. Atkin, T. Vuong, G. Batist, N. Beauchemin, D. Radzioch, S. Martel, Nature Nanotechnology 2016, 11, 941.

[85] T. Islam, C. Peng, I. Ali, Journal of Basic Microbiology 2018, 58, 378.

[86] S. Seong, T. H. Park, Biotechnology and Bioengineering 2001, 76, 11.

[87] Y. Wang, W. Lin, J. Li, Y. Pan, Frontiers in Microbiology 2013, 4.

[88] C. T. Lefèvre, T. Song, J.-P. Yonnet, L.-F. Wu, Appl. Environ. Microbiol. 2009, 75, 3835.

[89] I. S. M. Khalil, S. Misra, IEEE Trans. Magn. 2014, 50, 1.

[90] C. T. Lefèvre, M. L. Schmidt, N. Viloria, D. Trubitsyn, D. Schüler, D. A. Bazylinski, Applied and Environmental Microbiology 2012, 78, 7238.

[91] D. A. Bazylinski, R. B. Frankel, H. W. Jannasch, Nature 1988, 334, 518.

[92] C. L. Monteil, G. Perrière, N. Menguy, N. Ginet, B. Alonso, N. Waisbord, S. Cruveiller, D. Pignol, C. T. Lefèvre, Environmental Microbiology 2018, 20, 4415.

[93] T. J. Williams, C. T. Lefevre, W. Zhao, T. J. Beveridge, D. A. Bazylinski, INTERNATIONAL JOURNAL OF SYSTEMATIC AND EVOLUTIONARY MICROBIOLOGY 2012, 62, 2443. 


\section{WILEY-VCH}

[94] S. Bellini, Chinese Journal of Oceanology and Limnology 2009, 27, 3.

[95] R. Blakemore, Science 1975, 190, 377.

[96] R. B. Frankel, R. P. Blakemore, R. S. Wolfe, Science 1979, 203, 1355.

[97] R. Uebe, F. Ahrens, J. Stang, K. Jäger, L. H. Böttger, C. Schmidt, B. F. Matzanke, D. Schüler, mBio 2019, 10.

[98] S. Ullrich, M. Kube, S. Schubbe, R. Reinhardt, D. Schuler, Journal of Bacteriology 2005, 187, 7176.

[99] R. Uebe, D. Schüler, Nature Reviews Microbiology 2016, 14, 621.

[100] A. Scheffel, M. Gruska, D. Faivre, A. Linaroudis, J. M. Plitzko, D. Schüler, Nature 2006, 440, 110.

[101] M. Toro-Nahuelpan, G. Giacomelli, O. Raschdorf, S. Borg, J. M. Plitzko, M. Bramkamp, D. Schüler, F.-D. Müller, Nature Microbiology 2019, 4, 1978.

[102] H. C. McCausland, A. Komeili, PLOS Genetics 2020, 16, e1008499.

[103] F. Abreu, D. Acosta-Avalos, In Microorganisms [Working Title]; IntechOpen, 2018.

[104] B. L. Taylor, I. B. Zhulin, M. S. Johnson, Annu. Rev. Microbiol. 1999, 53, 103.

[105] T. Schweinitzer, C. Josenhans, Arch Microbiol 2010, 192, 507.

[106] S. Klumpp, C. T. Lefèvre, M. Bennet, D. Faivre, Physics Reports 2019, 789, 1.

[107] A. M. Spormann, R. S. Wolfe, FEMS Microbiology Letters 1984, 22, 171.

[108] D. C. Guell, H. Brenner, R. B. Frankel, H. Hartman, Journal of Theoretical Biology 1988, 135, 525.

[109] M. Bennet, A. McCarthy, D. Fix, M. R. Edwards, F. Repp, P. Vach, J. W. C. Dunlop, M. Sitti, G. S. Buller, S. Klumpp, D. Faivre, PLoS ONE 2014, 9, e101150.

[110] A. Codutti, K. Bente, D. Faivre, S. Klumpp, PLoS Comput Biol 2019, 15, e1007548.

[111] K. Bente, S. Mohammadinejad, M. A. Charsooghi, F. Bachmann, A. Codutti, C. T. Lefèvre, S. Klumpp, D. Faivre, High-speed motility originates from cooperatively pushing and pulling flagella bundles in bilophotrichous bacteria; Biophysics, 2019.

[112] S. Rismani Yazdi, R. Nosrati, C. A. Stevens, D. Vogel, P. L. Davies, C. Escobedo, Small 2018, 14, 1702982.

[113] S. Rismani Yazdi, R. Nosrati, C. A. Stevens, D. Vogel, C. Escobedo, Biomicrofluidics 2018, 12, 011101.

[114] F. Popp, J. P. Armitage, D. Schüler, Nature Communications 2014, 5.

[115] M. Mahmoudi, A. Tachibana, A. B. Goldstone, Y. J. Woo, P. Chakraborty, K. R. Lee, C. S. Foote, S. Piecewicz, J. C. Barrozo, A. Wakeel, B. W. Rice, C. B. Bell III, P. C. Yang, Scientific Reports 2016, 6 .

[116] M. Boucher, F. Geffroy, S. Prévéral, L. Bellanger, E. Selingue, G. Adryanczyk-Perrier, M. Péan, C. T. Lefèvre, D. Pignol, N. Ginet, S. Mériaux, Biomaterials 2017, 121, 167.

[117] M. Kuhara, H. Takeyama, T. Tanaka, T. Matsunaga, Analytical Chemistry 2004, 76, 6207.

[118] T. Yoshino, H. Hirabe, M. Takahashi, M. Kuhara, H. Takeyama, T. Matsunaga, Biotechnology and Bioengineering 2008, 101, 470.

[119] N. Ginet, R. Pardoux, G. Adryanczyk, D. Garcia, C. Brutesco, D. Pignol, PLoS ONE 2011, 6, e21442.

[120] I. Y. Iskusnykh, T. N. Popova, Biomeditsinskaya Khimiya 2010, 56, 530.

[121] R. Le Fèvre, M. Durand-Dubief, I. Chebbi, C. Mandawala, F. Lagroix, J.-P. Valet, A. Idbaih, C. Adam, J.-Y. Delattre, C. Schmitt, C. Maake, F. Guyot, E. Alphandéry, Theranostics 2017, 7, 4618.

[122] R. Liu, J. Liu, J. Tong, T. Tang, W.-C. Kong, X. Wang, Y. Li, J. Tang, Progress in Natural Science: Materials International 2012, 22, 31.

[123] A. Plan Sangnier, S. Preveral, A. Curcio, A. K. A. Silva, C. T. Lefèvre, D. Pignol, Y. Lalatonne, C. Wilhelm, Journal of Controlled Release 2018, 279, 271.

[124] J. Xu, L. Liu, J. He, S. Ma, S. Li, Z. Wang, T. Xu, W. Jiang, Y. Wen, Y. Li, J. Tian, F. Li, Journal of Nanobiotechnology 2019, 17. 


\section{WILEY-VCH}

[125] E. Alphandéry, D. Abi Haidar, O. Seksek, F. Guyot, I. Chebbi, Nanoscale 2018, 10, 10918.

[126] Y. Pan, N. Li, J. Mu, R. Zhou, Y. Xu, D. Cui, Y. Wang, M. Zhao, Applied Microbiology and Biotechnology 2015, 99, 703.

[127] J. J. Jacob, K. Suthindhiran, Biotechnology Reports 2020, 25, e00422.

[128] C.-Y. Chen, C.-F. Chen, Y. Yi, L.-J. Chen, L.-F. Wu, T. Song, Biomedical Microdevices 2014, 16, 761.

[129] M. R. Benoit, D. Mayer, Y. Barak, I. Y. Chen, W. Hu, Z. Cheng, S. X. Wang, D. M. Spielman, S. S. Gambhir, A. Matin, Clinical Cancer Research 2009, 15, 5170.

[130] S. Martel, M. Mohammadi, O. Felfoul, Zhao Lu, P. Pouponneau, The International Journal of Robotics Research 2009, 28, 571.

[131] J. El Fouladi, Z. Lu, Y. Savaria, S. Martel, In 2007 29th Annual International Conference of the IEEE Engineering in Medicine and Biology Society; IEEE: Lyon, France, 2007; pp. 119-122.

[132] D. Gandia, L. Gandarias, I. Rodrigo, J. Robles-García, R. Das, E. Garaio, J. Á. García, M. Phan, H. Srikanth, I. Orue, J. Alonso, A. Muela, M. L. Fdez-Gubieda, Small 2019, 15, 1902626.

[133] T. Matsunaga, F. Tadokoro, N. Nakamura, IEEE Transactions on Magnetics 1990, 26, 1557.

[134] S. Martel, In The First IEEE/RAS-EMBS International Conference on Biomedical Robotics and Biomechatronics, 2006. BioRob 2006.; IEEE: Pisa, Italy, 2006; pp. 829-834.

[135] M. M. Stanton, B.-W. Park, D. Vilela, K. Bente, D. Faivre, M. Sitti, S. Sánchez, ACS Nano 2017, $11,9968$.

[136] C. Chen, L. Chen, P. Wang, L.-F. Wu, T. Song, Journal of Magnetism and Magnetic Materials 2019, 479, 74.

[137] G. Harasko, H. Pfutzner, K. Futschik, IEEE Transactions on Magnetics 1995, 31, 938.

[138] A. S. Bahaj, I. W. Croudace, P. A. B. James, F. D. Moeschler, P. E. Warwick, Journal of Magnetism and Magnetic Materials 1998, 184, 241.

[139] A. S. Bahaj, D. C. Ellwood, J. H. P. Watson, IEEE Transactions on Magnetics 1991, 27, 5371.

[140] A. S. Bahaj, P. A. B. James, I. W. Croudace, IEEE Transactions on Magnetics 1994, 30, 4707.

[141] A. S. Bahaj, P. A. B. James, F. D. Moeschler, Journal of Applied Physics 1998, 83, 6444.

[142] J. A. Diaz-Alarcón, M. P. Alfonso-Pérez, I. Vergara-Gómez, M. Díaz-Lagos, S. A. MartínezOvalle, Journal of Environmental Management 2019, 249, 109381.

[143] B. A. Smit, E. Van Zyl, J. J. Joubert, W. Meyer, S. Prévéral, C. T. Lefèvre, S. N. Venter, Letters in Applied Microbiology 2018, 66, 362.

[144] N. Zeytuni, R. Uebe, M. Maes, G. Davidov, M. Baram, O. Raschdorf, A. Friedler, Y. Miller, D. Schüler, R. Zarivach, PLoS ONE 2014, 9, e97154.

[145] S. Martel, C. C. Tremblay, S. Ngakeng, G. Langlois, Applied Physics Letters 2006, 89, 233904.

[146] S. Martel, J.-B. Mathieu, O. Felfoul, A. Chanu, E. Aboussouan, S. Tamaz, P. Pouponneau, L. Yahia, G. Beaudoin, G. Soulez, M. Mankiewicz, Applied Physics Letters 2007, 90, 114105.

[147] T. Xu, J. Yu, X. Yan, H. Choi, L. Zhang, Micromachines 2015, 6, 1346.

[148] A. Ghanbari, P. H. Chang, B. J. Nelson, H. Choi, Smart Materials and Structures 2014, 23, 035013.

[149] I. S. M. Khalil, M. P. Pichel, L. Abelmann, S. Misra, The International Journal of Robotics Research 2013, 32, 637.

[150] I. S. M. Khalil, M. P. Pichel, B. A. Reefman, O. S. Sukas, L. Abelmann, S. Misra, In 2013 IEEE International Conference on Robotics and Automation; IEEE: Karlsruhe, Germany, 2013; pp. 5508-5513.

[151] Y. Zhang, N.-T. Nguyen, Lab Chip 2017, 17, 994.

[152] H. Wang, L. Chen, L. Sun, Front. Mech. Eng. 2017, 12, 510.

[153] S. Rismani Yazdi, P. Agrawal, E. Morales, C. A. Stevens, L. Oropeza, P. L. Davies, C. Escobedo, R. D. Oleschuk, Analytica Chimica Acta 2019, 1085, 107.

[154] C. J. Pierce, H. Wijesinghe, E. Osborne, E. Mumper, B. Lower, S. Lower, R. Sooryakumar, AIP Advances 2020, 10, 015335. 


\section{WILEY-VCH}

[155] Q. Li, H. Chen, X. Feng, C. Yu, F. Feng, Y. Chai, P. Lu, T. Song, X. Wang, L. Yao, Small 2019, 15, 1900427.

[156] B. J. Nelson, I. K. Kaliakatsos, J. J. Abbott, Annu. Rev. Biomed. Eng. 2010, 12, 55.

[157] K. C. Leptos, K. Y. Wan, M. Polin, I. Tuval, A. I. Pesci, R. E. Goldstein, Phys. Rev. Lett. 2013, $111,158101$.

[158] G. Santomauro, A. V. Singh, B. Park, M. Mohammadrahimi, P. Erkoc, E. Goering, G. Schütz, M. Sitti, J. Bill, Adv. Biosys. 2018, 2, 1800039.

[159] U. K. Cheang, D. Roy, J. H. Lee, M. J. Kim, Appl. Phys. Lett. 2010, 97, 213704.

[160] R. W. Carlsen, M. R. Edwards, J. Zhuang, C. Pacoret, M. Sitti, Lab Chip 2014, 14, 3850.

[161] H. Xu, M. Medina-Sánchez, V. Magdanz, L. Schwarz, F. Hebenstreit, O. G. Schmidt, ACS Nano 2018, 12, 327.

[162] C. Ridzewski, M. Li, B. Dong, V. Magdanz, ACS Appl. Bio Mater. 2020.

[163] V. Magdanz, M. Medina-Sánchez, L. Schwarz, H. Xu, J. Elgeti, O. G. Schmidt, Advanced Materials 2017, 29, 1606301.

[164] I. S. M. Khalil, S. Misra, In Microbiorobotics; Elsevier, 2017; pp. 61-79.

[165] H. A. Hassan, M. Pichel, T. Hageman, L. Abelmann, I. S. M. Khalil, In 2016 IEEE/RSJ International Conference on Intelligent Robots and Systems (IROS); IEEE: Daejeon, South Korea, 2016; pp. 5119-5124.

[166] J. Baumgartner, L. Bertinetti, M. Widdrat, A. M. Hirt, D. Faivre, PLoS ONE 2013, 8, e57070.

[167] D. M. S. ESQUIVEL, H. G. P. LINS DE BARROS, J. Exp. Biol. 1986, 121, 153.

[168] Y. Pan, W. Lin, L. Tian, R. Zhu, N. Petersen, Geomicrobiology Journal 2009, 26, 313.

[169] R. Nadkarni, S. Barkley, C. Fradin, PLoS ONE 2013, 8, e82064.

[170] E. Wajnberg, L. H. S. de Souza, H. G. P. L. de Barros, D. M. S. Esquivel, Biophysical Journal 1986, 50, 451 .

[171] M. Blondeau, Y. Guyodo, F. Guyot, C. Gatel, N. Menguy, I. Chebbi, B. Haye, M. Durand-Dubief, E. Alphandery, R. Brayner, T. Coradin, Scientific Reports 2018, 8.

[172] M. Naresh, K. Gopinadhan, S. Sekhar, P. Juneja, M. Sharma, A. Mittal, IEEE Transactions on Magnetics 2009, 45, 4861.

[173] C. Rosenblatt, F. F. T. de Araujo, R. B. Frankel, Journal of Applied Physics 1982, 53, 2727.

[174] C. Rosenblatt, F. F. T. de Araujo, R. B. Frankel, Biophysical Journal 1982, 40, 83.

[175] S. Taherkhani, M. Mohammadi, J. Daoud, S. Martel, M. Tabrizian, ACS Nano 2014, 8, 5049.

[176] Q. Ma, C. Chen, S. Wei, C. Chen, L.-F. Wu, T. Song, Biomicrofluidics 2012, 6, 024107.

[177] S. K. Alsaiari, A. H. Ezzedine, A. M. Abdallah, R. Sougrat, N. M. Khashab, OpenNano 2016, 1, 36.

[178] J. Cypriano, J. Werckmann, G. Vargas, A. Lopes dos Santos, K. T. Silva, P. Leão, F. P. Almeida, D. A. Bazylinski, M. Farina, U. Lins, F. Abreu, PLOS ONE 2019, 14, e0215657.

[179] A. Curcio, A. Van de Walle, A. Serrano, S. Preveral, C. Péchoux, D. Pignol, N. Menguy, C. T. Lefevre, A. Espinosa, C. Wilhelm, ACS Nano 2020, 14, 1406.

[180] R. Fernandes, M. Zuniga, F. R. Sassine, M. Karakoy, D. H. Gracias, Small 2011, 7, 588.

[181] O. I. Sentürk, O. Schauer, F. Chen, V. Sourjik, S. V. Wegner, Advanced Healthcare Materials 2020, 9, 1900956.

[182] R. Di Leonardo, L. Angelani, D. Dell'Arciprete, G. Ruocco, V. Iebba, S. Schippa, M. P. Conte, F. Mecarini, F. De Angelis, E. Di Fabrizio, Proceedings of the National Academy of Sciences 2010, $107,9541$.

[183] Y.-A. Chen, A. D. King, H.-C. Shih, C.-C. Peng, C.-Y. Wu, W.-H. Liao, Y.-C. Tung, Lab on a Chip 2011, 11, 3626.

[184] R. Alonso-Matilla, D. Saintillan, EPL (Europhysics Letters) 2018, 121, 24002.

[185] F. Guzmán-Lastra, A. Kaiser, H. Löwen, Nature Communications 2016, 7.

[186] F. R. Koessel, S. Jabbari-Farouji, EPL (Europhysics Letters) 2019, 125, 28001.

[187] F. Meng, D. Matsunaga, R. Golestanian, Physical Review Letters 2018, 120. 


\section{WILEY-VCH}

[188] B. Vincenti, G. Ramos, M. L. Cordero, C. Douarche, R. Soto, E. Clement, Nature Communications 2019, $10,1$.

[189] L. Sonntag, J. Simmchen, V. Magdanz, Molecules 2019, 24, 3410.

[190] M. Sun, X. Fan, X. Meng, J. Song, W. Chen, L. Sun, H. Xie, Nanoscale 2019, 11, 18382.

[191] K. Kamata, Z. Piao, S. Suzuki, T. Fujimori, W. Tajiri, K. Nagai, T. Iyoda, A. Yamada, T. Hayakawa, M. Ishiwara, S. Horaguchi, A. Belay, T. Tanaka, K. Takano, M. Hangyo, Scientific Reports 2015, 4, 4919.

[192] X. Yan, Q. Zhou, M. Vincent, Y. Deng, J. Yu, J. Xu, T. Xu, T. Tang, L. Bian, Y.-X. J. Wang, K. Kostarelos, L. Zhang, Science Robotics 2017, 2, eaaq1155.

[193] X. Li, J. Cai, L. Sun, Y. Yue, D. Zhang, RSC Advances 2016, 6, 76716.

[194] S. K. Srivastava, M. Medina-Sánchez, B. Koch, O. G. Schmidt, Advanced Materials 2015, 28, 832.

[195] V. Magdanz, J. Gebauer, D. Mahdi, J. Simmchen, I. K. M. Khalil, In Manipulation, Automation and Robotics at Small Scales (MARSS), International Conference on; IEEE: Helsinki, 2019.

[196] I. S. M. Khalil, V. Magdanz, J. Simmchen, A. Klingner, S. Misra, Applied Physics Letters 2020, 116, 63702.

[197] V. Magdanz, S. Sanchez, O. G. Schmidt, Advanced Materials 2013, 25, 6581.

[198] I. S. M. Khalil, V. Magdanz, S. Sanchez, O. G. Schmidt, S. Misra, Journal of Micro-Bio Robotics 2014, 9, 79.

[199] V. Magdanz, M. Medina-Sánchez, Y. Chen, M. Guix, O. G. Schmidt, Advanced Functional Materials 2015, 25, 2763.

[200] V. Magdanz, M. Guix, F. Hebenstreit, O. G. Schmidt, Advanced Materials 2016, 28, 4048.

[201] H. Xu, M. Medina-Sánchez, V. Magdanz, L. Schwarz, F. Hebenstreit, O. G. Schmidt, ACS Nano 2018, 12, 327.

[202] C. Ridzewski, M. Li, B. Dong, V. Magdanz, ACS Appl. Bio Mater. 2020.

[203] M. Medina-Sánchez, L. Schwarz, A. K. Meyer, F. Hebenstreit, O. G. Schmidt, Nano letters 2015, $16,555$.

[204] V. Magdanz, I. S. M. Khalil, J. Simmchen, G. P. Furtado, S. Mohanty, J. Gebauer, H. Xu, A. Klingner, A. Aziz, M. Medina-Sánchez, O. G. Schmidt, S. Misra, Science Advances 2020.

[205] R. M. Harshey, Annual Review of Microbiology 2003, 57, 249.

[206] J. Bastos-Arrieta, A. Revilla-Guarinos, W. E. Uspal, J. Simmchen, Frontiers in Robotics and AI 2018, 5, 97.

[207] Y. Alapan, O. Yasa, B. Yigit, I. C. Yasa, P. Erkoc, M. Sitti, Annual Review of Control, Robotics, and Autonomous Systems 2019, 2, 205.

[208] V. Du Nguyen, J.-W. Han, Y. J. Choi, S. Cho, S. Zheng, S. Y. Ko, J.-O. Park, S. Park, Sensors and Actuators B: Chemical 2016, 224, 217.

[209] D. Li, H. Choi, S. Cho, S. Jeong, Z. Jin, C. Lee, S. Y. Ko, J. O. Park, S. Park, Biotechnology and Bioengineering 2015, 112, 1623.

[210] O. Schauer, B. Mostaghaci, R. Colin, D. Hürtgen, D. Kraus, M. Sitti, V. Sourjik, Scientific Reports 2018, 8, 9801.

[211] D. Kim, A. Liu, E. Diller, M. Sitti, Biomedical Microdevices 2012, 14, 1009.

[212] O. Felfoul, M. Mohammadi, S. Taherkhani, D. de Lanauze, Y. Zhong Xu, D. Loghin, S. Essa, S. Jancik, D. Houle, M. Lafleur, L. Gaboury, M. Tabrizian, N. Kaou, M. Atkin, T. Vuong, G. Batist, N. Beauchemin, D. Radzioch, S. Martel, Nature Nanotechnology 2016, 11, 941.

[213] Z. Lu, S. Martel, In Engineering in Medicine and Biology Society, 2006. EMBS'06. 28th Annual International Conference of the IEEE; IEEE, 2006; pp. 3415-3418.

[214] S. Martel, M. Mohammadi, O. Felfoul, Z. Lu, P. Pouponneau, The International Journal of Robotics Research 2009, 28, 571.

[215] J. Zhuang, R. W. Carlsen, M. Sitti, Scientific reports 2015, 5, 11403.

[216] B.-W. Park, J. Zhuang, O. Yasa, M. Sitti, ACS Nano 2017, 11, 8910. 


\section{WILEY-VCH}

[217] Á. Barroso, S. Landwerth, M. Woerdemann, C. Alpmann, T. Buscher, M. Becker, A. Studer, C. Denz, Biomedical microdevices 2015, 17, 26.

[218] M. M. Stanton, B.-W. Park, A. Miguel-López, X. Ma, M. Sitti, S. Sánchez, Small 2017, 13, 1603679.

[219] M. M. Stanton, J. Simmchen, X. Ma, A. Miguel-López, S. Sánchez, Advanced Materials Interfaces 2016, 3.

[220] R. W. Carlsen, M. R. Edwards, J. Zhuang, C. Pacoret, M. Sitti, Lab on a Chip 2014, 14, 3850.

[221] O. Yasa, P. Erkoc, Y. Alapan, M. Sitti, Advanced Materials 2018, 30, 1804130.

[222] X. Yan, Q. Zhou, J. Yu, T. Xu, Y. Deng, T. Tang, Q. Feng, L. Bian, Y. Zhang, A. Ferreira, L. Zhang, Advanced Functional Materials 2015, 25, 5333.

[223] I. Boldea, S. A. Nasar, IEEE Transactions on Energy Conversion 1999, 14, 712.

[224] R. C. Chiechi, E. A. Weiss, M. D. Dickey, G. M. Whitesides, Angewandte Chemie International Edition 2008, 47, 142.

[225] M. D. Dickey, R. C. Chiechi, R. J. Larsen, E. A. Weiss, D. A. Weitz, G. M. Whitesides, Advanced Functional Materials 2008, 18, 1097.

[226] G. J. Hayes, J.-H. So, A. Qusba, M. D. Dickey, G. Lazzi, IEEE Transactions on Antennas and Propagation 2012, 60, 2151.

[227] B.-H. Jo, L. M. Van Lerberghe, K. M. Motsegood, D. J. Beebe, Journal of microelectromechanical systems 2000, 9, 76.

[228] A. Mata, A. J. Fleischman, S. Roy, Biomedical microdevices 2005, 7, 281.

[229] T. N. Do, H. Phan, T.-Q. Nguyen, Y. Visell, Advanced Functional Materials 2018, 28, 1800244.

[230] M. Amjadi, Y. J. Yoon, I. Park, Nanotechnology 2015, 26, 375501.

[231] K. O. Siegenthaler, A. Künkel, G. Skupin, M. Yamamoto, In Synthetic biodegradable polymers; Springer, 2011; pp. 91-136.

[232] M. Yamamoto, U. Witt, G. Skupin, D. Beimborn, R.-J. Müller, Biopolymers Online: Biology• Chemistry• Biotechnology•Applications 2005, 4.

[233] J. O’Connor, J. Punch, N. Jeffers, J. Stafford, Microfluidics and Nanofluidics 2015, 19, 385.

[234] D. P. Parekh, C. Ladd, L. Panich, K. Moussa, M. D. Dickey, Lab on a Chip 2016, 16, 1812.

[235] S. Dottermusch, D. Busko, M. Langenhorst, U. W. Paetzold, B. S. Richards, Optics letters 2019, 44, 29.

[236] D. B. Fullager, G. D. Boreman, T. Hofmann, Optical Materials Express 2017, 7, 888.

[237] J. G. Rider, C. T. B. Foxon, The Philosophical Magazine: A Journal of Theoretical Experimental and Applied Physics 1967, 16, 1133.

[238] Y.-H. Lin, S.-W. Kang, T.-Y. Wu, Applied Thermal Engineering 2009, 29, 573.

[239] B. Slusarek, K. Zakrzewski, Przegl. Elektrotech.(Electr. Rev.) 2012, 88, 123.

[240] L. A. Dobrzański, M. Drak, B. Ziębowicz, Journal of Achievements in Materials and Manufacturing Engineering 2006, 17, 37.

[241] J. Kaleta, S. Tumański, J. Żebracki, Journal of magnetism and magnetic materials 1996, 160, 199.

[242] W. Fang, I. Panagiotopoulos, F. Ott, F. Boué, K. Ait-Atmane, J.-Y. Piquemal, G. Viau, F. Dalmas, Journal of nanoparticle research 2014, 16, 2265.

[243] M. De Cuyper, P. Müller, H. Lueken, M. Hodenius, Journal of Physics: Condensed Matter 2003, $15, \mathrm{~S} 1425$.

[244] N. Ebrahimi, P. Schimpf, A. Jafari, Sensors and Actuators A: Physical 2018, 284, 276.

[245] M. Ziegler, K. Ochs, M. Hansen, H. Kohlstedt, Applied Physics A 2014, 114, 565.

[246] S. Odenbach, Ferrofluids: magnetically controllable fluids and their applications; Springer, 2008; Vol. 594.

[247] H. Hay, Organisation of magnetic microparticles in a ferrofluid: a computational approach to a self-assembly process; 2012.

[248] A. Marette, A. Poulin, N. Besse, S. Rosset, D. Briand, H. Shea, Advanced Materials 2017, 29, 1700880. 


\section{WILEY-VCH}

[249] J. J. Rassweiler, R. Autorino, J. Klein, A. Mottrie, A. S. Goezen, J.-U. Stolzenburg, K. H. Rha, M. Schurr, J. Kaouk, V. Patel, BJU international 2017, 120, 822.

[250] B. Mosadegh, A. D. Mazzeo, R. F. Shepherd, S. A. Morin, U. Gupta, I. Z. Sani, D. Lai, S. Takayama, G. M. Whitesides, Lab on a Chip 2014, 14, 189.

[251] K.-Y. Shin, J.-Y. Hong, J. Jang, Chemical communications 2011, 47, 8527.

[252] J. Zhang, S. Yan, D. Yuan, G. Alici, N.-T. Nguyen, M. E. Warkiani, W. Li, Lab on a Chip 2016, $16,10$.

[253] D. Rus, M. T. Tolley, Nature 2015, 521, 467.

[254] R. Guo, L. Sheng, H. Gong, J. Liu, Science China Technological Sciences 2018, 61, 516.

[255] R. M. McKenzie, M. E. Sayed, M. P. Nemitz, B. W. Flynn, A. A. Stokes, Soft robotics 2019, 6, 195.

[256] M. P. Nemitz, P. Mihaylov, T. W. Barraclough, D. Ross, A. A. Stokes, Soft robotics 2016, 3, 198.

[257] B. Zhang, Y. Fan, P. Yang, T. Cao, H. Liao, Soft robotics 2019, 6, 399.

[258] A. Jafari, N. Ebrahimi, Electromagnetic soft actuators 2020.

[259] N. Ebrahimi, S. Nugroho, A. F. Taha, N. Gatsis, W. Gao, A. Jafari, In 2018 IEEE International Conference on Robotics and Automation (ICRA); IEEE, 2018; pp. 2857-2864.

[260] L. Sliker, G. Ciuti, M. Rentschler, A. Menciassi, Expert Review of Medical Devices 2015, 12.

[261] J. J. Abbott, E. Diller, A. J. Petruska, Annual Review of Control, Robotics, and Autonomous Systems 2020, 3, annurev.

[262] R. D. Brewer, K. E. Loewke, E. F. Duval, J. K. Salisbury, In Proceedings of the 2nd Biennial IEEE/RAS-EMBS International Conference on Biomedical Robotics and Biomechatronics, BioRob 2008; 2008; pp. 580-586.

[264] G. Ciuti, P. Valdastri, A. Menciassi, P. Dario, Robotica 2010, 28, 199.

[265] G. Ciuti, R. Donlin, P. Valdastri, A. Arezzo, A. Menciassi, M. Morino, P. Dario, Endoscopy 2010, 42.

[266] A. Arezzo, A. Menciassi, P. Valdastri, G. Ciuti, G. Lucarini, M. Salerno, C. Di Natali, M. Verra, P. Dario, M. Morino, Digestive and Liver Disease 2013, 45.

[267] A. W. Mahoney, J. J. Abbott, Applied Physics Letters 2011, 99, 134103.

[268] A. W. Mahoney, J. J. Abbott, IEEE Transactions on Robotics 2014, 30, 411.

[269] T. L. Bruns, K. E. Riojas, D. S. Ropella, M. S. Cavilla, A. J. Petruska, M. H. Freeman, R. F. Labadie, J. J. Abbott, R. J. Webster, IEEE Robotics and Automation Letters 2020, 5, 2240.

[270] S. E. Wright, A. W. Mahoney, K. M. Popek, J. J. Abbott, In IEEE Transactions on Robotics; Institute of Electrical and Electronics Engineers Inc., 2017; Vol. 33, pp. 1013-1024.

[271] A. W. Mahoney, J. J. Abbott, The International Journal of Robotics Research 2016, 35, 129.

[272] L. J. Sliker, G. Ciuti, Expert Review of Medical Devices 2014, 11, 649.

[273] N. Shamsudhin, V. I. Zverev, H. Keller, S. Pane, P. W. Egolf, B. J. Nelson, A. M. Tishin, Magnetically guided capsule endoscopy: Medical Physics 2017, 44, e91-e111.

[274] G. Ciuti, R. Caliò, D. Camboni, L. Neri, F. Bianchi, A. Arezzo, A. Koulaouzidis, S. Schostek, D. Stoyanov, C. M. Oddo, B. Magnani, A. Menciassi, M. Morino, M. O. Schurr, P. Dario, Journal of Micro-Bio Robotics 2016, 11, 1.

[275] F. Carpi, S. Galbiati, A. Carpi, Biomedicine and Pharmacotherapy 2006, 60, 370.

[276] F. Carpi, S. Galbiati, A. Carpi, IEEE Transactions on Biomedical Engineering 2007, 54, 2028.

[277] F. Carpi, C. Pappone, Biomedicine and Pharmacotherapy 2008, 62, 546.

[278] F. Carpi, C. Pappone, IEEE Transactions on Biomedical Engineering 2009, 56, 1482.

[279] F. Carpi, C. Pappone, Stereotaxis Niobe ${ }^{\circledR}$ magnetic navigation system for endocardial catheter ablation and gastrointestinal capsule endoscopy. Expert Review of Medical Devices 2009, 6, 487498.

[280] F. Carpi, N. Kastelein, M. Talcott, C. Pappone, IEEE Transactions on Biomedical Engineering 2011, 58, 231. 


\section{WILEY-VCH}

[281] J. Keller, C. Fibbe, F. Volke, J. Gerber, A. C. Mosse, M. Reimann-Zawadzki, E. Rabinovitz, P. Layer, P. Swain, Gastrointestinal Endoscopy 2010, 72, 941.

[282] J. Keller, C. Fibbe, F. Volke, J. Gerber, A. C. Mosse, M. Reimann-Zawadzki, E. Rabinovitz, P. Layer, D. Schmitt, V. Andresen, U. Rosien, P. Swain, Gastrointestinal Endoscopy 2011, 73, 22.

[283] G. S. Lien, C. W. Liu, J. A. Jiang, C. L. Chuang, M. T. Teng, IEEE Transactions on Biomedical Engineering 2012, 59, 2068.

[284] Z. Li, Z. Liao, M. McAlindon, Handbook of capsule endoscopy; Springer Netherlands, 2014.

[285] I. Rahman, M. Pioche, C. S. Shim, S. P. Lee, I. K. Sung, J. C. Saurin, P. Patel, Gastrointestinal Endoscopy 2016, 83, 889.

[286] J. F. Rey, H. Ogata, N. Hosoe, K. Ohtsuka, N. Ogata, K. Ikeda, H. Aihara, I. Pangtay, T. Hibi, S. Kudo, H. Tajiri, Endoscopy 2010, 42, 541.

[287] J. F. Rey, H. Ogata, N. Hosoe, K. Ohtsuka, N. Ogata, K. Ikeda, H. Aihara, I. Pangtay, T. Hibi, S. E. Kudo, H. Tajiri, Gastrointestinal Endoscopy 2012, 75, 373.

[288] U. W. Denzer, T. Rösch, B. Hoytat, M. Abdel-Hamid, X. Hebuterne, G. Vanbiervielt, J. Filippi, H. Ogata, N. Hosoe, K. Ohtsuka, N. Ogata, K. Ikeda, H. Aihara, S. E. Kudo, H. Tajiri, A. Treszl, K. Wegscheider, M. Greff, J. F. Rey, Journal of Clinical Gastroenterology 2015, 49, 101.

[289] X. Dong, M. Sitti, The International Journal of Robotics Research 2020, 39, 617.

[290] D. Loghin, C. Tremblay, M. Mohammadi, S. Martel, The International Journal of Robotics Research 2017, 36, 1195.

[291] M. P. Kummer, J. J. Abbott, B. E. Kratochvil, R. Borer, A. Sengul, B. J. Nelson, IEEE Transactions on Robotics 2010, 26, 1006.

[292] L. Dong, B. J. Nelson, IEEE Robotics and Automation Magazine 2007, 14, 111.

[293] J. J. Abbott, Z. Nagy, F. Beyeler, B. J. Nelson, IEEE Robotics and Automation Magazine 2007, $14,92$.

[294] X. Jiang, J. Pan, Z. S. Li, Z. Liao, VideoGIE 2019, 4, 239.

[295] Z. Liao, X. Hou, E. Q. Lin-Hu, J. Q. Sheng, Z. Z. Ge, B. Jiang, X. H. Hou, J. Y. Liu, Z. Li, Q. Y. Huang, X. J. Zhao, N. Li, Y. J. Gao, Y. Zhang, J. Q. Zhou, X. Y. Wang, J. Liu, X. P. Xie, C. M. Yang, H. L. Liu, X. T. Sun, W. Bin Zou, Z. S. Li, Clinical Gastroenterology and Hepatology 2016, $14,1266$.

[296] C. S. Cheng, T. J. Sun, H. de Zhang, BMC gastroenterology 2019, 19, 184.

[297] P. Valdastri, G. Ciuti, A. Verbeni, A. Menciassi, P. Dario, A. Arezzo, M. Morino, Surgical Endoscopy and Other Interventional Techniques 2012, 26, 1238.

[298] L. J. Sliker, G. Ciuti, M. E. Rentschler, A. Menciassi, Tribology International 2016, 102.

[299] J. Li, E. S. Barjuei, G. Ciuti, Y. Hao, P. Zhang, A. Menciassi, Q. Huang, P. Dario, Journal of Magnetism and Magnetic Materials 2018, 452.

[300] M. Salerno, G. Ciuti, G. Lucarini, R. Rizzo, P. Valdastri, A. Menciassi, A. Landi, P. Dario, Measurement Science and Technology 2012, 23, 015701.

[301] A. Taddese, P. Slawinski, ... M. P.-... J. of R., undefined 2018, eprints.whiterose.ac.uk.

[302] F. Bianchi, A. Masaracchia, E. Shojaei Barjuei, A. Menciassi, A. Arezzo, A. Koulaouzidis, D. Stoyanov, P. Dario, G. Ciuti, Expert Review of Medical Devices 2019, 16, 381.

[303] G. Ciuti, M. Salerno, G. Lucarini, P. Valdastri, A. Arezzo, A. Menciassi, M. Morino, P. Dario, IEEE Transactions on Robotics 2012, 28.

[304] P. R. Slawinski, N. Simaan, A. Z. Taddese, K. L. Obstein, P. Valdastri, IEEE Transactions on Robotics 2019, 35, 1123.

[305] L. Barducci, G. Pittiglio, J. C. Norton, K. L. Obstein, P. Valdastri, IEEE Robotics and Automation Letters 2019, 4, 3633.

[306] P. R. Slawinski, A. Z. Taddese, K. B. Musto, S. Sarker, P. Valdastri, K. L. Obstein, Gastroenterology 2018, 154, 1577.

[307] J. C. Norton, P. R. Slawinski, H. S. Lay, J. W. Martin, B. F. Cox, G. Cummins, M. P. Y. Desmulliez, R. E. Clutton, K. L. Obstein, S. Cochran, P. Valdastri, Science Robotics 2019, 4. 


\section{WILEY-VCH}

[309] F. Bianchi, G. Ciuti, A. Koulaouzidis, A. Arezzo, D. Stoyanov, S. Schostek, C. M. Oddo, A. Menciassi, P. Dario, An innovative robotic platform for magnetically-driven painless colonoscopy. Annals of Translational Medicine 2017, 5.

[310] P. Brandao, E. Mazomenos, G. Ciuti, R. Caliò, F. Bianchi, A. Menciassi, P. Dario, A. Koulaouzidis, A. Arezzo, D. Stoyanov, Armato, S. G.; Petrick, N. A., Eds.; 2017; p. 101340F.

[311] P. Brandao, O. Zisimopoulos, E. Mazomenos, G. Ciuti, J. Bernal, M. Visentini-Scarzanella, A. Menciassi, P. Dario, A. Koulaouzidis, A. Arezzo, D. J. Hawkes, D. Stoyanov, Journal of Medical Robotics Research 2018, 03, 1840002.

[312] Di. K. Iakovidis, G. DImas, A. Karargyris, F. Bianchi, G. Ciuti, A. Koulaouzidis, IEEE Journal of Biomedical and Health Informatics 2019, 23, 2211.

[313] M. Visentini-Scarzanella, H. Kawasaki, R. Furukawa, M. Bonino, S. Arolfo, G. Lo Secco, A. Arezzo, A. Menciassi, P. Dario, G. Ciuti, Endoscopy International Open 2018, 06, E602.

[314] G. Dimas, F. Bianchi, D. K. Iakovidis, A. Karargyris, G. Ciuti, A. Koulaouzidis, Measurement Science and Technology 2020.

G. Lucarini, M. Mura, G. Ciuti, R. Rizzo, A. Menciassi, Journal of Medical and Biological Engineering 2015, 35, 428.

[317] G. Lucarini, G. Ciuti, M. Mura, R. Rizzo, A. Menciassi, International Journal of Advanced Robotic Systems 2015, 12.

[318] S. Nouda, K. Ota, K. Higuchi, Digestive Endoscopy 2018, 30, 117.

[319] D. Son, H. Gilbert, M. Sitti, Soft Robotics 2020, 7, 10.

[320] K. T. Nguyen, M. C. Hoang, E. Choi, B. Kang, J. O. Park, C. S. Kim, International Journal of Control, Automation and Systems 2020, 18, 65.

[321] K. Kim, X. Liu, Y. Zhang, Y. Sun, J. Micromech. Microeng. 2008, 18, 055013.

[322] Z. Zhang, X. Wang, J. Liu, C. Dai, Y. Sun, Annual Review of Control, Robotics, and Autonomous Systems 2019, 2, 181.

[323] M. P. Kummer, J. J. Abbott, B. E. Kratochvil, R. Borer, A. Sengul, B. J. Nelson, IEEE Transactions on Robotics 2010, 26, 1006.

[324] H. Xie, M. Sun, X. Fan, Z. Lin, W. Chen, L. Wang, L. Dong, Q. He, Science Robotics 2019, 4.

[325] J. Li, T. Li, T. Xu, M. Kiristi, W. Liu, Z. Wu, J. Wang, Nano Lett. 2015, 15, 4814.

[326] S. Palagi, A. G. Mark, S. Y. Reigh, K. Melde, T. Qiu, H. Zeng, C. Parmeggiani, D. Martella, A. Sanchez-Castillo, N. Kapernaum, F. Giesselmann, D. S. Wiersma, E. Lauga, P. Fischer, Nat Mater 2016, 15, 647.

[327] S. Fournier-Bidoz, A. C. Arsenault, I. Manners, G. A. Ozin, Chem. Commun. 2005, 441.

[328] W. Wang, L. A. Castro, M. Hoyos, T. E. Mallouk, ACS Nano 2012, 6, 6122.

[329] J. S. Randhawa, T. G. Leong, N. Bassik, B. R. Benson, M. T. Jochmans, D. H. Gracias, J. Am. Chem. Soc. 2008, 130, 17238.

[330] T. G. Leong, C. L. Randall, B. R. Benson, N. Bassik, G. M. Stern, D. H. Gracias, Proc. Natl. Acad. Sci. U.S.A. 2009, 106, 703.

[331] S. Fusco, M. S. Sakar, S. Kennedy, C. Peters, S. Pane, D. Mooney, B. J. Nelson, In 2014 IEEE International Conference on Robotics and Automation (ICRA); 2014; pp. 3777-3782.

[332] E. Diller, M. Sitti, Advanced Functional Materials 2014, 24, 4377.

[333] J. Zhang, O. Onaizah, K. Middleton, L. You, E. Diller, IEEE Robotics and Automation Letters 2017, 2, 835 .

[334] I. S. M. Khalil, F. van den Brink, O. S. Sukas, S. Misra, In 2013 IEEE International Conference on Robotics and Automation; 2013; pp. 5527-5532.

[335] A. E. El-Etriby, A. Klingner, A. F. Tabak, I. S. M. Khalil, In 2018 International Conference on Manipulation, Automation and Robotics at Small Scales (MARSS); 2018; pp. 1-4. 


\section{WILEY-VCH}

[336] P. J. Burke, In Encyclopedia of Nanoscience and Nanotechnology; H. S. Nalwa), American Scientific Publishers: Eds, 2004; Vol. 10, pp. 1-19.

[337] K. C. Neuman, S. M. Block, Review of Scientific Instruments 2004, 75, 2787.

[338] H. Becker, L. E. Locascio, Talanta 2002, 56, 267.

[339] J. Shi, D. Ahmed, X. Mao, S.-C. S. Lin, A. Lawit, T. J. Huang, Lab Chip 2009, 9, 2890.

[340] Akira Masuda, Takeshi Yamakawa, Lev Zimin, In 2008 SICE Annual Conference; 2008; pp. 1747-1750.

[341] K.-F. Böhringer, V. Bhatt, B. R. Donald, K. Goldberg, Algorithmica 2000, 26, 389.

[342] H. Lee, A. M. Purdon, R. M. Westervelt, Appl. Phys. Lett. 2004, 85, 1063.

[343] S. Floyd, C. Pawashe, M. Sitti, IEEE Transactions on Robotics 2009, 25, 1332.

[344] C. Pawashe, S. Floyd, E. Diller, M. Sitti, IEEE Transactions on Robotics 2012, 28, 467.

[345] K. E. Peyer, L. Zhang, B. J. Nelson, Appl. Phys. Lett. 2011, 99, 174101.

[346] T. Petit, L. Zhang, K. E. Peyer, B. E. Kratochvil, B. J. Nelson, Nano Lett. 2012, 12, 156.

[347] M. A. Seif, A. Hassan, A. H. El-Shaer, A. Alfar, S. Misra, I. S. M. Khalil, In 2017 IEEE International Conference on Advanced Intelligent Mechatronics (AIM); 2017; pp. 1095-1102.

[348] Z. Ye, E. Diller, M. Sitti, Journal of Applied Physics 2012, 112, 064912.

[349] A. G. El-Gazzar, L. E. Al-Khouly, A. Klingner, S. Misra, I. S. M. Khalil, In 2015 IEEE/RSJ International Conference on Intelligent Robots and Systems (IROS); 2015; pp. 778-783.

[350] H.-W. Tung, K. E. Peyer, D. F. Sargent, B. J. Nelson, Appl. Phys. Lett. 2013, 103, 114101.

[351] I. S. M. Khalil, A. Klingner, Y. Hamed, Y. S. Hassan, S. Misra, IEEE Trans. Robot. 2020.

[352] I. S. M. Khalil, A. F. Tabak, Y. Hamed, M. E. Mitwally, M. Tawakol, A. Klingner, M. Sitti, Advanced Science 2018, 5, 1700461.

[353] C. Hu, S. Pané, B. J. Nelson, Annual Review of Control, Robotics, and Autonomous Systems 2018, $1,53$.

[354] J. Hwang, J. Kim, H. Choi, Intelligent Service Robotics 2020, 13, 1.

[355] E. P. Furlani, Permanent magnet and electromechanical devices: materials, analysis, and applications; Academic press, 2001.

[356] V. Iacovacci, G. Lucarini, C. Innocenti, N. Comisso, P. Dario, L. Ricotti, A. Menciassi, Biomedical Microdevices 2015, 17, 112.

[357] K. Tsuchida, H. M. García-García, W. J. van der Giessen, E. P. McFadden, M. van der Ent, G. Sianos, H. Meulenbrug, A. T. L. Ong, P. W. Serruys, Catheterization and Cardiovascular Interventions 2006, 67, 356.

[358] S. Jeon, A. K. Hoshiar, K. Kim, S. Lee, E. Kim, S. Lee, J. Kim, B. J. Nelson, H.-J. Cha, B.-J. Yi, H. Choi, Soft Robotics 2018, 6, 54.

[359] C. Chautems, A. Tonazzini, Q. Boehler, S. H. Jeong, D. Floreano, B. J. Nelson, Advanced Intelligent Systems n/a, 1900086.

[360] V. Lalande, F. P. Gosselin, S. Martel, In 2010 Annual International Conference of the IEEE Engineering in Medicine and Biology; 2010; pp. 1874-1877.

[361] F. P. Gosselin, V. Lalande, S. Martel, Medical Physics 2011, 38, 4994.

[362] V. Lalande, F. P. Gosselin, M. Vonthron, B. Conan, C. Tremblay, G. Beaudoin, G. Soulez, S. Martel, Medical Physics 2015, 42, 969.

[363] A. Azizi, C. C. Tremblay, K. Gagné, S. Martel, Science Robotics 2019, 4, eaax7342.

[364] A. D. Losey, P. Lillaney, A. J. Martin, D. L. Cooke, M. W. Wilson, B. R. H. Thorne, R. S. Sincic, R. L. Arenson, M. Saeed, S. W. Hetts, Radiology 2014, $271,862$.

[365] Y. Kim, G. A. Parada, S. Liu, X. Zhao, Science Robotics 2019, 4, eaax7329.

[366] O. Vermesh, A. Aalipour, T. J. Ge, Y. Saenz, Y. Guo, I. S. Alam, S. Park, C. N. Adelson, Y. Mitsutake, J. Vilches-Moure, E. Godoy, M. H. Bachmann, C. C. Ooi, J. K. Lyons, K. Mueller, H. Arami, A. Green, E. I. Solomon, S. X. Wang, S. S. Gambhir, Nature Biomedical Engineering 2018, 2, 696. 


\section{WILEY-VCH}

[367] V. Iacovacci, L. Ricotti, E. Sinibaldi, G. Signore, F. Vistoli, A. Menciassi, Advanced Science 2018, $5,1800807$.

[368] V. Iacovacci, L. Ricotti, G. Signore, F. Vistoli, E. Sinibaldi, A. Menciassi, In 2019 International Conference on Robotics and Automation (ICRA); 2019; pp. 2495-2501. 